\title{
FROM INTERSTELLAR POLYCYCLIC AROMATIC HYDROCARBONS AND ICE TO ASTROBIOLOGY
}

\author{
LOUIS J. ALLAMANDOLA AND DOUGLAS M. HUDGINS \\ Astrochemistry Laboratory, NASA Ames Research Center, \\ MS 245-6, Mountain View, CA 94035-1000, USA
}

\begin{abstract}
Tremendous strides have been made in our understanding of interstellar material over the past twenty years thanks to significant, parallel developments in observational astronomy and laboratory astrophysics. Twenty years ago the composition of interstellar dust was largely guessed at, the concept of ices in dense molecular clouds ignored, and the notion of large, abundant, gas phase, carbon rich molecules widespread throughout the interstellar medium (ISM) considered impossible. Today the composition of dust in the diffuse ISM is reasonably well constrained to micron-sized cold refractory materials comprised of amorphous and crystalline silicates mixed with an amorphous carbonaceous material containing aromatic structural units and short, branched aliphatic chains. In dense molecular clouds, the birthplace of stars and planets, these cold dust particles are coated with mixed molecular ices whose major components are is very well constrained. Lastly, the signature of carbon-rich polycyclic aromatic hydrocarbons (PAHs), shockingly large molecules by earlier interstellar chemistry standards, is widespread throughout the Universe. This paper presents a detailed summary of these disparate interstellar components and ends by considering both of them as important feedstock to the chemical inventory of the primordial Earth. Particular attention is paid to their possible role in the chemistry that led to the origin of life. An extensive reference list is given to allow the student entry into the full depth of the literature.

The first part of this paper focuses on interstellar PAHs. The laboratory and theoretical underpinning which supports the PAH model is reviewed in some detail. This is followed by a few specific examples which demonstrate how these data can be used to analyze the interstellar spectra and probe local conditions in different emission zones. These examples include tracing the evolution of carbon as it passes from its birthsite in circumstellar shells through the ISM, determining specifics about the cosmic PAH population in many different environments including PAH size and structure, and probing local conditions in the different emission zones.

The second part of this paper summarizes the laboratory and observational background leading to our current understanding of interstellar/precometary ices. Although the most abundant interstellar ice components are the very simple molecules such as $\mathrm{H}_{2} \mathrm{O}, \mathrm{CH}_{3} \mathrm{OH}, \mathrm{CO}, \mathrm{CO}_{2}$, and $\mathrm{NH}_{3}$, more complex species including accreted PAHs and those formed by UV and cosmic ray processing within the ice must also be present. Here we give a detailed summary of the photochemical evolution on those ices
\end{abstract}


found in the densest regions of molecular clouds, the regions where stars and planetary systems are formed. Ultraviolet photolysis of these ices produces a host of new compounds, some of which show intriguing prebiotic behavior.

The last part of this paper draws all this information together and considers the possible roles these compounds might have played in early Earth chemistry. As interstellar ices are the building blocks of comets and comets are thought to be an important source of the species which fell on the primitive Earth, their composition may be related to the origin of life. Three potential roles are considered, ranging from these interstellar materials simply providing the raw materials used for a completely endoginous origin to the opposite extreme that they delivered species poised to take part in the life process, or perhaps even at the earliest stages of what would be perceived of as a living system.

\section{Introduction}

The origin of life on earth is intimately tied to the nonbiological formation and chemical evolution of the compounds of carbon with five elements: $\mathrm{H}, \mathrm{N}, \mathrm{O}, \mathrm{S}$, and P. Since the Sun and planets formed some 4.6 billion years ago in a Universe perhaps 15 20 billion years of age, these elements have had a long and complex history before incorporation into the pre-solar nebula. Although it was once believed that the chemical compounds in space are rather simple, with chemical complexity limited by harsh radiation fields and extremely low densities, recent research has begun to call this belief into question. Today, compelling evidence is mounting that a substantial fraction of the carbon incorporated into planets, their satellites, asteroids, and comets in developing planetary systems is in the form of complex organic molecules. Thus, it is entirely possible that the extraterrestrial evolution of chemical complexity may play a crucial perhaps even a determinant - role in defining the early, prebiotic chemical state of these planetary systems. Moreover, the relative cosmic abundances of $\mathrm{O}, \mathrm{C}$, and $\mathrm{N}$ illustrated in Figure 1 underscore the fact that (ignoring the chemically inert helium) these elements are by far the most abundant chemically reactive elements after hydrogen, dwarfing the amounts of the next tier of elements including that of silicon. This implies that if life exists anywhere else in the cosmos, it is most likely composed of these most abundant elements and therefore evinces a chemistry similar to our own.

The history of the biogenic elements (e.g. C, N, and O) begins with their nucleosynthesis deep within the interiors of late-type stars. These elements are dredged up and thrown off into the surrounding interstellar medium (ISM) during the periods of intense mass-loss that punctuate the late asymptotic giant branch (AGB) phase. For carbon-rich AGB stars, extensive observational studies have shown that a wide array of organic materials are formed during these episodes of late-stellar mass-loss. Included in these outflows are simple molecules ( $<20$ atoms) such as acetylene, carbon monoxide, and the polyacetylenic and cyanopolyacetylenic chains; large, robust molecules (tens to hundreds of atoms) such as polycyclic aromatic hydrocarbons (PAHs); and small (100$1000 \AA$ A, several thousands of atoms or more) amorphous carbon and silicate dust particles. As this ejecta gradually disperses through the protoplanetary and planetary nebula (PPN and PNe) stages, the surrounding diffuse ISM is gradually enriched with these carbonaceous materials. In the diffuse ISM, these compounds and particles are further modified through a variety of physical and chemical processes including: UV 


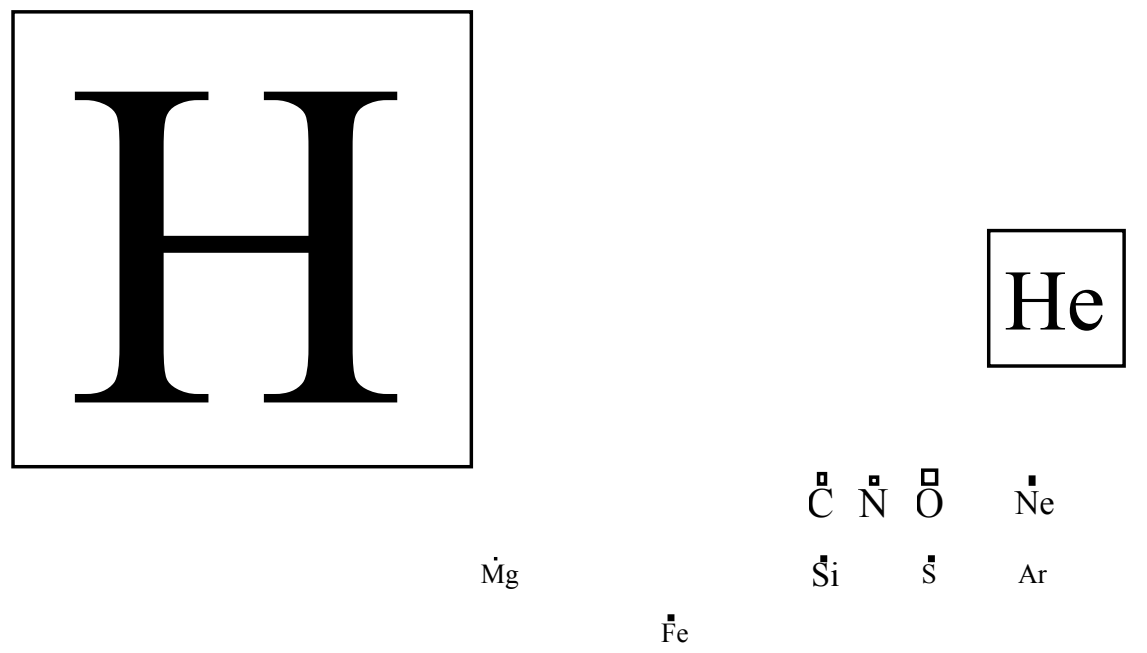

Figure 1. Astronomer's periodic table of the elements with the areas of the boxes illustrating the relative cosmic abundances of the most plentiful elements. Figure reproduced courtesy of Ben McCall.

irradiation; cosmic ray bombardment; gas-phase chemistry; accretion and reaction upon grain surfaces; and destruction by shock waves generated by supernova explosions. Numerous reviews relevant to this wide range of phenomena can be found elsewhere in this volume and in the scientific literature.[1,2]

While refractory dust particles and large molecular species such as PAHs are relatively immune to photodestruction in the diffuse ISM, the simpler polyatomic molecules cannot withstand the harsh interstellar UV radiation field and quickly dissociate. Fortuitously for us, the existence of interstellar matter is not limited to the tenuous, optically-thin medium that permeates our galaxy. On the contrary, much material is also concentrated in large, relatively opaque interstellar molecular clouds.[3,4,5] Within these 'dark' clouds, the interstellar ultraviolet radiation field is strongly attenuated, permitting simple, fragile molecules to form and flourish through a rich network of gas phase reactions. Although a wide variety of these simple molecular species have been identified in the gas-phase by extensive radio and infrared observations (e.g. $[4,6,7]$ ), one should bear in mind that these represent only one aspect of the chemical inventory of these regions. In fact, at the low temperatures which characterize these dark molecular clouds $(\approx 10-50 \mathrm{~K})$, the majority of molecular species are expected to be frozen out upon the surfaces of cold, refractory grains (e.g. [8]). Thus, the molecular inventory of cold, dark interstellar clouds must be shaped by a combination of ion-molecule reactions in the gas phase and gas-grain surface reactions (e.g. [9,10]). Moreover, the attenuated diffuse ISM UV which penetrates a dense cloud, as well as UV from internal sources and penetrating cosmic ray particles are sufficient to drive in-situ, solid-state reactions within the icy grain mantles leading to a variety of even more complex species.

It is within cold, dark molecular clouds such as this that new stars and planetary systems are born. Once part of a molecular cloud becomes unstable under its own gravitational field, it will begin to collapse, forming a protostar. As this collapse 
proceeds, the angular momentum possessed by the infalling material draws it into a disk. Planetary systems are thought to coalesce from the remnants of this protostellar accretion disk after the young star springs to life (the "Solar Nebula"). Thus, the raw material from which planetary systems form contains the biogenic elements in the same diverse states of molecular complexity found in the parent molecular cloud. Naturally, this biogenic material may also be modified to some extent by chemistry taking place during the collapse as well as in the accretion shock. Chemical processes at work during this epoch include equilibrium gas-phase reactions in the warmer regions of the nebula and non-equilibrium processes in colder regions and on coalescing planetessimals. Ultimately, the biogenic compounds present, whether produced in the nebula or accepted unchanged from the ISM, are incorporated into the condensed matter that became the planets, satellites, asteroids, and comets. Thus, the compounds that emerge from the interstellar/protostellar crucible provide the seed from which life must spring, and the study of these organic compounds is crucial to our understanding of the origin and early evolution of life.

Once a terrestrial planet is sufficiently cool to retain volatile materials, comets and meteoritic materials continuously pepper it with copious quantities of their complex organic inventory (e.g. [11]). Consequently, from the standpoint of Astrobiology, it is important to understand both the nature of the large reservoir of complex, carbon-rich materials in the ISM, as well as the composition and chemistry encountered in cold interstellar ice grains - the building blocks of comets. Both of these sources can be much more chemically complex than the gaseous interstellar material. Furthermore, since icy grain mantles in all likelihood represent the largest repository of interstellar molecules in dense clouds, they tie up a large fraction of the biogenic elements available in these clouds. Given the likelihood that a substantial amount of material was delivered to the early earth through the influx of cometary matter, and in light of the molecular complexity now known to characterize such ices, it is reasonable to postulate that such materials may have played more than just a "spectators" role in the origin of life.

In this paper we review the foundations and summarize the current knowledge of the most complex organic molecules that comprise this extraterrestrial primordial soup. In section 2, the basis for the presence of PAHs in the ISM will be presented, followed by an overview of the laboratory studies that have been carried out to verify and refine the model. The salient astrophysical implications of this laboratory work are presented and insights into the size distribution, structure, and ionization state as a function of interstellar object type are drawn based on the cutting-edge astronomical data returned by the Infrared Space Observatory (ISO). The following section ( $\$ 3$ ) focuses on the evidence for interstellar/precometary ices and presents a summary of the laboratory work that has led to our current understanding of interstellar ice composition. The photochemical evolution of these ices in the absence and in the presence of PAHs is then considered $(\$ 4)$ with an eye toward the abiotic production of biologically active compounds. Finally, in the last section ( $\$ 5)$, the possible contribution of this chemistry to the chemistry of the primordial Earth and the origin of life is considered. 


\section{INTERSTELLAR PAHS: THE LARGEST INTERSTELLAR MOLECULES}

\subsection{INTERSTELLAR PAHS: THE OBSERVATIONAL FOUNDATION}

A discrete emission feature at $11.3 \mu \mathrm{m}$ discovered by Gillett, Forrest, and Merrill in 1973 [12] was the first member of a now-well-known family of interstellar emission bands to be reported. Subsequent observations by a host of observers (c.f. [13-16]) revealed that this was just one element in a recurring set of prominent emission bands at $3.3,6.2,7.7$, and $8.6 \mu \mathrm{m}$ as well as a complex array of minor bands, plateaus, and underlying continua. This spectrum is now known to be an integral part of the IR emission from many different astronomical objects including H II regions (Figure 2), planetary nebulae, reflection nebulae, and the ISM of our galaxy as well as that of other galaxies.[17] Moreover, these features carry $20-40 \%$ of the total IR luminosity from many of these galactic objects [18], indicating that the carrier represents an abundant and ubiquitous component of the ISM. Calculations show that the carriers of these features are as abundant as the most abundant simple polyatomic molecule, $\mathrm{NH}_{3}\left(10^{-6}-10^{-7}\right.$ with respect to hydrogen; e.g.[19,20]). Remarkably, recent ISO observations have even indicated that these features are sufficiently prevalent and intense in distant galaxies that they might be useful as red-shift indicators (e.g. [21]).

It was first proposed in the mid-1980's that this widespread emission spectrum might be diagnostic of gas phase polycyclic aromatic hydrocarbon $(\mathrm{PAH})$ molecules and closely related species.[19,20] The rationale underlying this suggestion is straightforward. First, the emission bands are non-thermal in nature - that is, they are observed even in regions where the dust temperature is too low for the material to be emitting thermally.[19,20,23-25] Thus, the emission must be excited by the absorption of individual UV/visible photons, implying that the carriers are free, gas phase molecules rather than a solid state material. Second, there is a direct correlation between
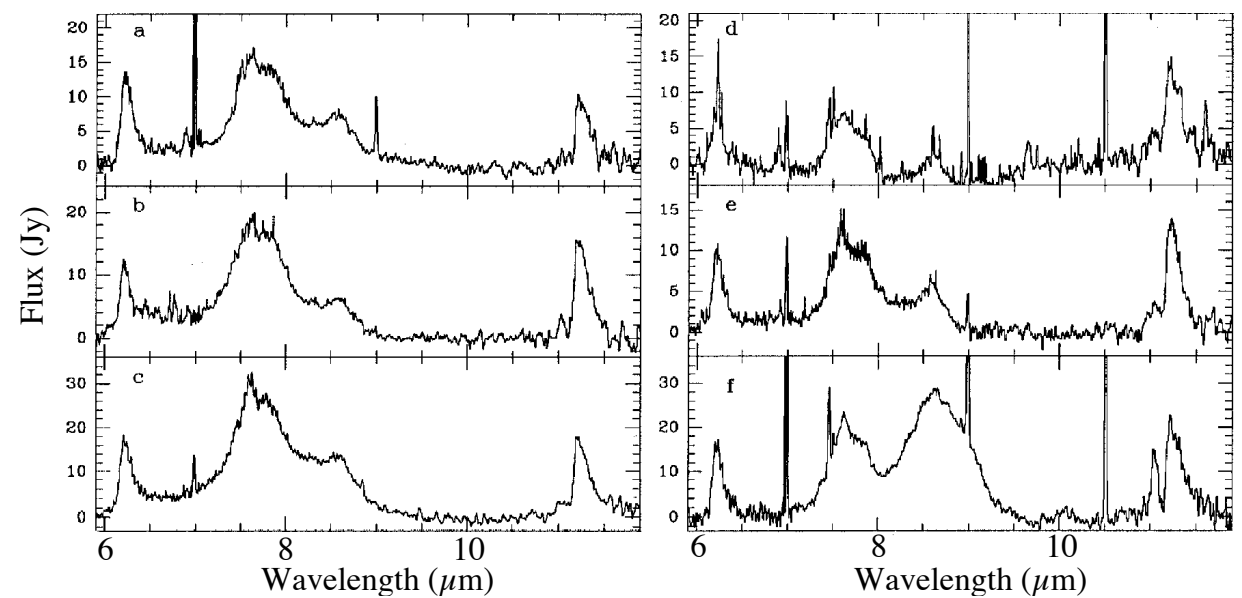

Figure 2. The $6-12 \mu \mathrm{m}$ spectra of six compact HII regions illustrating the appearance of the common interstellar emission features at $6.2,7.7,8.6$, and $11.2 \mu \mathrm{m}$. The sources are IRAS: (a) $8116-1646$; (b) $18162-2048$; (c) $19442-2427$; (d) $21190+5140$; (e) $22308+5812$; and (f) $18434-$ 0242. Figure adapted from [22]. 
carbon abundance and the intensity of the emission features [26,27], implying that the gas phase carriers are carbon-based molecules. Third, the emission features are observed even from extremely harsh environments, indicating that the gaseous, carbon-rich molecules are exceptionally stable. Finally, the positions of the interstellar emission features provide insight into the chemical nature of the material from which they originate. Significantly, prominent bands in the interstellar emission spectrum fall at all the positions that would be expected to arise from the vibrational transitions of aromatic molecules. Taken together, these elements provide strong evidence that polycyclic aromatic hydrocarbons are prevalent in the ISM.

Obviously, the existence of a previously-unsuspected, yet very abundant interstellar material such as this could have important ramifications in many areas of astrophysics, and a number of new perspectives on long-standing problems were born in the years following the introduction of the PAH model. For example, since the ubiquity and complexity of the PAH population reflect a chemical history very different from the traditional ion-molecule interstellar chemistry, they were posited as the molecular "missing link" to larger carbonaceous dust grains.[20] Indeed, observations are beginning to support this picture.[28] Others suggested that PAHs could fundamentally influence temperature [29,30], and ionization level, chemistry, and radiative transfer [31] within molecular clouds, thereby influencing star formation; that interstellar PAHs may have been the source of much of the carbon in meteorites and interplanetary dust particles [32-35]; and that PAHs in one form or another might account for some of the enigmatic visible/near-IR diffuse interstellar bands (DIBs) [36-38], a very long-standing interstellar puzzle. Comprehensive discussions of the PAH model as it stood at the end of the 1980's can be found in the literature $[39,40]$ and a more thorough discussion of the widespread evidence for PAHs can be found in reference [41]. The purpose of the remainder of this section is to focus exclusively on the information contained in the IR spectra of PAHs and how this information is used as a probe of conditions in many different interstellar environments.

\subsection{INTERSTELLAR PAHS: THE LABORATORY CHALLENGE}

To more effectively test and exploit the PAH hypothesis and to capitalize on the wealth of astronomical IR spectral data now available thanks to the orbiting and airborne telescopes requires data on the physical, spectral, and chemical properties of PAHs in their likely interstellar forms: i.e. free, gas phase neutral molecules, ions, dehydrogenated or super-hydrogenated radicals, and clusters.[19,20,39,40] Beyond simply reflecting the physical characteristics of the emission zones, combining such data with the latest observations provides a unique window on the chemical evolution of carbonaceous material throughout the ISM. For example, significant spectral variations have been found between objects of a particular class (e.g. Figure 2) and between the various classes of IR emitting objects (protoplanetary nebulae, planetary nebulae, HII regions, reflection nebulae, etc), implying that different PAH populations are favored in different regions.[42-47] Further,. Comprehensive astrophysical models based on such observations hold the promise to provide insight into the chemical make-up of different objects; to trace the chemical evolution of those objects as they change from one stage to another; and to probe the ionization balance and other conditions within the emission zones over the wide range of objects which emit the features. Nevertheless, to gain this valuable insight, such models require a thorough understanding of the spectroscopic 
properties of PAHs, and the interplay between PAH vibronic states and their associated radiative photoprocesses with the interstellar radiation field - fundamental molecular information which can only be obtained through appropriate laboratory experiments.

Unfortunate in this regard, early testing and exploitation of the PAH hypothesis was severely hampered by a general lack of knowledge of the spectroscopic properties of PAHs under astrophysically relevant conditions. At the time of its inception, the laboratory data available to the PAH model were limited primarily to spectra measured from pure PAH crystals, from PAHs dispersed in salt pellets or organic solvents, or from PAHs embedded in glassy melts. Under such conditions, the individual PAH molecules are not effectively isolated and interact strongly with each other and/or with the surrounding medium. These conditions strongly influence the measured spectra and are far from the cold, isolated conditions encountered in the ISM. Furthermore - and in retrospect, most importantly - all of the then-available spectroscopic data were for neutral PAHs, while the I/S PAHs were, instead, believed to be ionized. Consequently, all that could be said by the end of the 1980's was that the interstellar spectra "resembled" the spectra of some neutral PAHs.

Be that as it may, it is one thing to say that one needs astrophysically relevant spectroscopic data, but it is quite another to actually realize that goal in the laboratory. Within the framework of the PAH model, the interstellar infrared emission arises from transiently-heated, gas-phase PAHs in both neutral and ionized forms. The goal of laboratory work in this area has been to measure the spectroscopic properties of these species under conditions which approximate those encountered in the ISM. At first glance, it might seem that spectral measurements of gas phase PAHs might easily be undertaken through the use of a simple single- or multipass gas cell, suitably modified for the study of these compounds. Specifically, since all but the smallest PAHs have vanishingly low vapor pressures at room temperature, the gas cell would have to be heated to facilitate warming the sample within to achieve a measurable vapor pressure. Indeed, in this manner it has been possible to measure the absorption and emission spectra of a few species and these studies have produced important insights into the astrophysical problem.[48-52] Nevertheless, this technique faces a number of serious practical difficulties for all but the very smallest PAHs. Moreover, these difficulties become increasingly problematic as molecular size increases and the volatility of the sample decreases. Since the optical surfaces of the cell cannot be heated as effectively as the rest of the cell, for PAHs larger than about 14 carbon atoms the pure material slowly but inexorably crystallizes out on these cooler surfaces over the course of an experiment. As this happens, the overall throughput of the cell gradually drops and the spectrum of the solid PAH becomes increasingly prominent until it completely dominates the measured spectrum.

Another problem one encounters in laboratory studies of PAHs centers on the overall efficiency of the experimental technique - that is, the amount of spectroscopic data one can collect per mass of PAH sample consumed. This is of concern because many PAHs - particularly the large PAHs thought to be important in the ISM - are often obtainable only in milligram quantities and/or at great expense. Furthermore, clean-up after an experiment will entail exposure to the often carcinogenic/mutagenic PAH material and disposal of the resulting potentially hazardous waste. The standard gas-cell technique is particularly inefficient because it requires a relatively large sample of the solid PAH to fill a cell with PAH vapor and maintain it against crystallization, and because recovery of the spent material after the experiment is usually not practical. 
What's more, even if one should overcome all of these difficulties, one would only have succeeded in studying neutral PAHs. To study ionized PAHs one must overcome all of these difficulties plus wrestle with the problem of generating and maintaining a measurable population of PAH ions under these conditions.

Finally, experimental challenges aside, perhaps the most insidious problem associated with undertaking astrophysically relevant spectroscopic studies of PAHs in the laboratory stems from the counterintuitive photophysics of the interstellar emission process. In a conventional laboratory sample of PAH vapor, generated by appropriate warming of a solid PAH, the individual PAH molecules are fully thermalized at the vaporization temperature, $\mathrm{T}_{\text {vap }}$, i.e. $\mathrm{T}_{\mathrm{vap}} \approx \mathrm{T}_{\text {vib }} \approx \mathrm{T}_{\text {rot }}$, where $\mathrm{T}_{\text {rot }}$ and $\mathrm{T}_{\text {vib }}$ are the rotational and vibrational temperatures of the molecules which reflect the internal energy content of their respective modes. Typically, even for modestly sized PAHs, $\mathrm{T}_{\mathrm{vap}}$ is on the order of a few hundred Kelvin or higher and the observed infrared bands are heavily broadened as the spectral intensity for each vibrational transition is distributed over an extensive manifold of rotational substates. This is not, however, the case for the interstellar PAHs. In the ISM, the average time between excitation events is typically far longer than that required for radiative relaxation. Thus, the PAH molecules are not in thermal equilibrium with their surroundings, instead spending the majority of their time in a fully rotationally and vibrationally relaxed state at an effective temperature of perhaps only $\sim 100 \mathrm{~K}$. When a fully relaxed species such as this absorbs a UV/visible photon, the initial electronic excitation energy is almost immediately converted to vibrational excitation, and the effective vibrational temperature of the molecule skyrockets. However, under the collisionless conditions of the ISM, angular momentum conservation prevents the redistribution of any internal energy into rotations. Thus, the effective rotational temperature of the molecule remains low. As a result, in contrast to the broad, double-humped rovibrational envelopes observed in the laboratory spectrum, the interstellar emission bands display a much narrower profile resulting from essentially pure vibrational transitions.[39,53,54] Thus, while the early gas-phase measurements discussed above provided an important milestone and test of the PAH hypothesis, further testing and exploitation of the model necessitated that other tools be brought to bear on the problem.

Faced with these limitations, verification and advancement of the PAH model has catalyzed the development of other creative experimental procedures that reduce or eliminate the difficulties associated with the straightforward, heated gas-cell method described above. These include both gas phase and solid-state, astrophysically relevant spectroscopic techniques. In the Astrochemistry Laboratory at NASA Ames a major effort has been underway for the last decade directed towards addressing the need for astrophysically-relevant, laboratory spectroscopic data on PAHs from the ultraviolet $(190 \mathrm{~nm})$ through the far-infrared $(200 \mu \mathrm{m})$. Here we will focus on the results of our IR studies of PAHs as this is where the astronomical and laboratory databases are most extensive and most directly relevant to the astrobiological issues discussed in $\S 3$. Recent excellent reviews of the current research into the spectroscopy and astrophysical impact of PAHs in the ultraviolet/visible region of the spectrum can be found elsewhere.[55,56]

To overcome the practical experimental difficulties outlined above, we have employed the matrix isolation technique to generate a database of infrared spectra of $\mathrm{PAHs}$ and $\mathrm{PAH}$ ions. In this technique, $\mathrm{PAH}$ vapor is generated by warming a sample of the solid in a small test tube mounted on a high vacuum chamber. The vapor effuses from the tube and is co-condensed with an overabundance of an inert gas (typically argon 
for infrared studies) onto a cryogenically cooled $(10 \mathrm{~K})$ infrared window suspended within the vacuum chamber. In this highly diluted sample, each PAH molecule is isolated from its neighbors and interacts only very weakly with the inert matrix, resulting in a quasi-gas phase condition. Furthermore, this condition can be maintained indefinitely as long as the window temperature is maintained. Cold PAH ions are generated by subsequent in-situ UV photolysis of the matrix-isolated neutral species and their spectral features distinguished from that of the neutral by comparison of spectra measured before and after photolysis. More complete discussions of the matrix isolation technique and the various experimental methods that have been employed to generate and study the IR spectral characteristics of neutral PAHs, PAH cations, and PAH anions can be found elsewhere.[57,58,59] Among its advantages, this technique is extremely efficient. Essentially all of the vaporized material that exits the reservoir tube is incorporated into the matrix-isolated sample. Since only $\sim 100-200 \mu \mathrm{g}$ of matrix-isolated PAH are required for an experiment, PAH samples of only a few milligrams are sufficient for many experiments. Clean-up is also greatly simplified, entailing minimal waste and exposure hazard, since the PAH residue remaining after the experiment is small and confined to the sample window. Additionally, although the matrix-isolation technique is limited to the measurement of absorption spectra, it faithfully reproduces the low rotational temperatures of interstellar PAHs, and careful modeling together with the latest experimental studies of jet-cooled, gas phase PAHs have shown that a simple thermal model is adequate for calculating of the astrophysical emission spectrum of PAHs based on their absorption spectra.[54,60,61] Finally, regarding the fidelity of argon matrix-isolated vibrational spectra relative to their corresponding gas phase spectra, over 40 years of matrix isolation research has shown that for most species, infrared band positions typically fall within 5 to $10 \mathrm{~cm}^{-1}(\lesssim 1 \%$ ) of their corresponding gas phase values. Theoretical and gas phase experimental studies have specifically corroborated this accuracy for PAHs and their ions.

Using the matrix-isolation technique, we have generated a spectral database which includes the infrared spectra of over 100 neutral, cationic, and anionic PAHs ranging in size from $\mathrm{C}_{10} \mathrm{H}_{8}$ to $\mathrm{C}_{48} \mathrm{H}_{20}$. [57,58,62-71] The species currently included in the database are listed in Table 1. Amongst the species currently represented in the dataset are: (1) the thermodynamically most stable PAHs through coronene, $\mathrm{C}_{24} \mathrm{H}_{12}$, the molecules most likely to be amongst the smallest interstellar PAHs; (2) a representative sampling of species from the fluoranthene family, aromatic hydrocarbons which incorporate a fivemembered ring in their carbon skeleton; (3) dicoronylene, $\mathrm{C}_{48} \mathrm{H}_{20}$, the largest $\mathrm{PAH}$ studied to date; and (4) a variety of "aza-PAHs", polycyclic aromatic compounds with a nitrogen atom incorporated into their carbon skeleton. We have also begun the process of making these data available to the scientific community on the internet at <http://web99.arc.nasa.gov/ astrochm/pahdata/index.html>. As discussed in detail in the next section, this data, together with that deriving from similar experimental studies conducted by Vala and coworkers at the University of Florida [72-79] and extensive theoretical studies [80-91] has established that mixtures of free molecular PAHs, dominated by PAH ions, can account for the global appearance of the interstellar emission spectra and the variations of those spectra. 


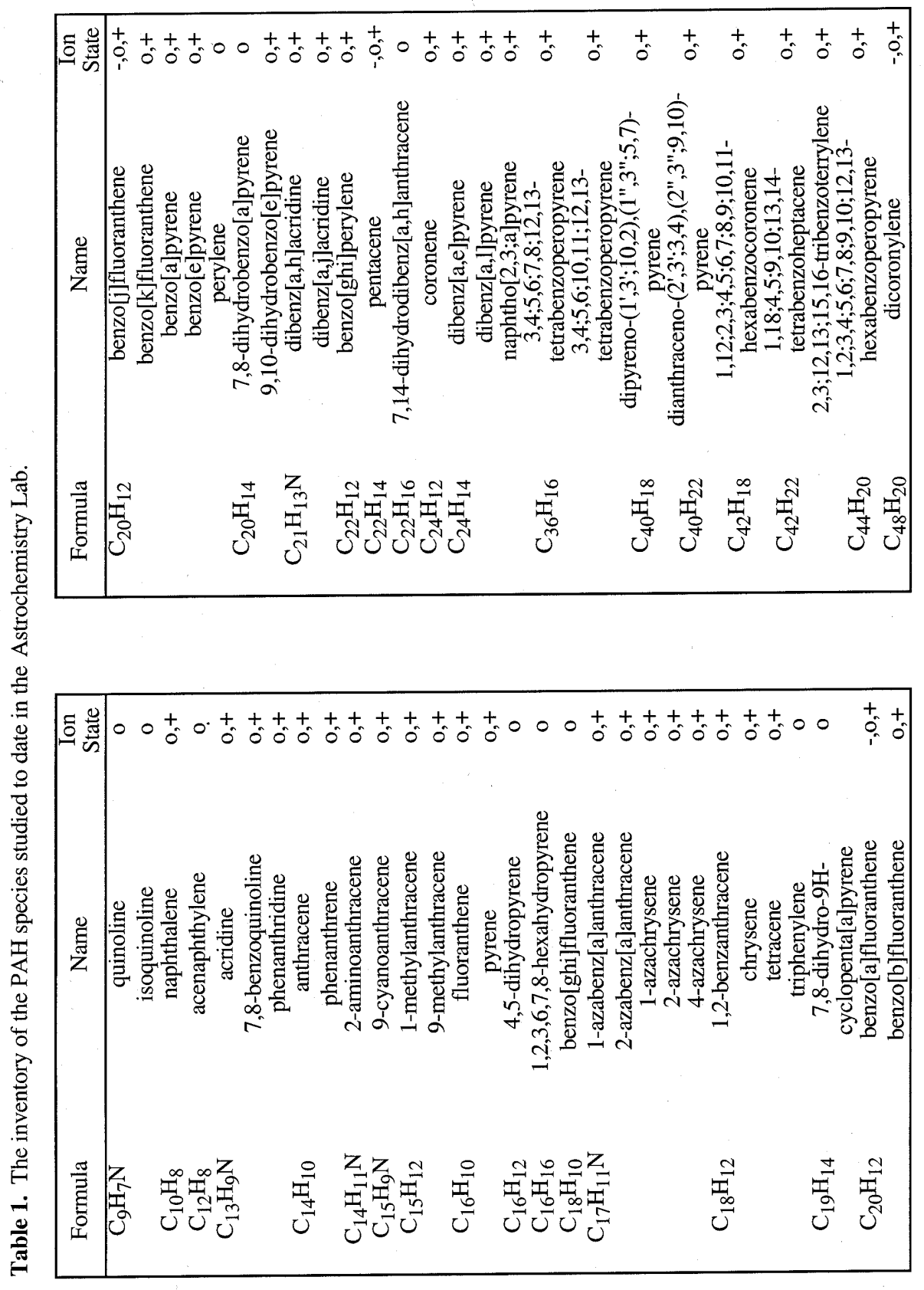


The culmination of all this work is the exploitation of PAHs as probes of local conditions in objects that span all stages of the lifecycle of interstellar matter. This entails quantitative comparisons of experimentally-measured infrared spectra of PAHs and PAH-related materials with the observed interstellar infrared emission spectra with the goal of gaining insight into the physical and chemical conditions within those emitting regions. Through comprehensive astrophysical modeling based on sound experimentally-measured spectroscopic properties, it will be possible to understand circumstellar dust formation and the entire evolutionary cycle of interstellar carbon-rich compounds from their production in late-stellar outflows to their incorporation into interstellar dust and new stellar systems. This information will further our knowledge of the role PAHs play in the chemical and dynamic evolution of interstellar clouds and the process of star formation.

Finally, we would be remiss if we were to fail to point out the emergence of two elegant new experimental techniques which permit direct spectroscopic measurements of gas-phase PAHs under conditions which are directly relevant to the astrophysical problem. These studies provide an important assessment of the astrophysical utility of the matrix-isolation spectral database and provide direct insight into the photophysics of the interstellar emission process. First, working at the Netherlands National Free Electron Laser Facility, Meijer and coworkers have developed a technique for directly measuring the infrared absorption spectrum of cold, gas phase PAH cations. In this technique, a jet cooled beam of PAH cation - inert gas atom van der Waals clusters is probed with the tunable infrared beam of a free electron laser. Infrared absorptions by the PAH cation precipitate dissociation of the fragile van der Waals clusters and are detected as a reduction in total cluster flux. Using this technique, Piest et al. have succeeded in measuring the IR spectrum of the naphthalene and phenanthrene cations in the gas phase.[92,93] Of direct interest here is the notable agreement between the infrared spectra obtained using this technique and those measured for the matrix isolated cations. This agreement is illustrated in Table 2 which compares the results of the two techniques for the naphthalene cation, $\mathrm{C}_{10} \mathrm{H}_{8}{ }^{+}$. Inspection of the table reveals that the majority of the argon matrix isolated vibrational band frequencies fall within $4 \mathrm{~cm}^{-1}$ $(\sim 0.4 \%)$ of the gas phase values determined using the cluster dissociation method (only one band differs by as much $8 \mathrm{~cm}^{-1}$ ). Such a modest matrix shift is negligible in the

Table 2. The frequencies and relative intensities for the cation of the PAH naphthalene $\left(\mathrm{C}_{10} \mathrm{H}_{8}\right)$ recently measured in the gas phase compared to the values measured using matrix isolation. The excellent agreement between the matrix and gas phase data for this PAH cation validates the matrix isolation approach to this problem.

\begin{tabular}{|cc|cc|cc|}
\hline $\begin{array}{c}\text { Gas Phase } \\
\text { Freq. }\left(\mathrm{cm}^{-1}\right)\end{array}$ & $\begin{array}{c}\text { Relative } \\
\text { Intensity }\end{array}$ & $\begin{array}{c}\text { Matrix } \\
\text { Freq. }\left(\mathrm{cm}^{-1}\right)\end{array}$ & $\begin{array}{c}\text { Relative } \\
\text { Intensity }\end{array}$ & $\begin{array}{c}\text { Matrix } \\
\text { Freq. }\left(\mathrm{cm}^{-1}\right)\end{array}$ & $\begin{array}{c}\text { Relative } \\
\text { Intensit } \\
\mathrm{y}\end{array}$ \\
\hline 589 & 0.04 & --- & --- & \\
759 & 0.05 & 758.7 & 0.27 & --- & 0.2 \\
1019 & 0.11 & $1016 / 1023.2$ & 0.05 & 1016 & \\
1121 & 0.01 & --- & & --- & 1.0 \\
1168 & 0.08 & --- & 0.2 & $1215 / 1218$ & \\
1215 & 1.0 & 1214.9 & 1.0 & --- & 0.04 \\
1284 & 0.01 & --- & & 1401 & 0.16 \\
1393 & 0.23 & 1400.9 & 0.04 & $1519 / 1525$ & \\
1523 & 0.4 & $1518.8 / 1525.7$ & 0.39 & & \\
1539 & 0.14 & & & & \\
\hline
\end{tabular}

a data taken from [92]; ${ }^{\mathrm{b}}$ data taken from [57]; ${ }^{\mathrm{c}}$ data taken from [72] 
context of the astrophysical problem, where the natural linewidth of a vibrationally excited PAH emitting under interstellar conditions is $\sim 30 \mathrm{~cm}^{-1}$. This result reinforces the validity of the use of matrix isolation data for the analysis of the interstellar infrared emission spectra.

Second, working at the University of California, Berkeley, Saykally and coworkers have directly measured the infrared emission from cold, vibrationally excited gas phase PAHs, shedding light on the photophysics of the interstellar UV/vis-to-IR conversion process. $[61,94,95]$ This work has been critically needed both to provide a demonstration of the infrared fluorescent emission mechanism and to test the validity of using the absorption properties of matrix-isolated PAHs to calculate the emission from vibrationally excited PAHs. Although these too are extremely challenging experiments, the infrared fluorescent emission from a number of neutral PAHs and the pyrene cation has successfully been measured under conditions identical to that of the I/S emitters. These studies have revealed heretofore unknown details of the emission process and support the quantum-/statistical-mechanical, molecular excitation-emission process put forward to calculate the emission of vibrationally excited PAHs and the use of matrixisolation spectra. This approach has been used in many models to describe how PAH molecules convert interstellar UV-visible radiation into IR.[39,40,96] In summary, gasphase and matrix-isolation laboratory studies of PAH spectra represent complementary rather than redundant aspects of the experimental work needed, each addressing different, critical aspects of the astrophysical problem.

\subsection{INTERSTELLAR PAHS: MID-INFRARED SPECTRAL PROPERTIES}

One of the early important results of all the laboratory and theoretical studies on neutral and ionized PAHs is the remarkably dramatic effect ionization has on their infrared spectra.[cf. 57,58,62-91,97] This effect is illustrated in Figure 3. The infrared spectra of neutral PAHs are dominated by strong features arising from aromatic $\mathrm{CH}$ stretching vibrations near $3.3 \mu \mathrm{m}$ and $\mathrm{CH}$ out-of-plane bending vibrations between 11 and $14 \mu \mathrm{m}$. Weaker features arising from aromatic $\mathrm{CC}$ stretching and $\mathrm{CH}$ in-plane bending vibrations are observed in the 6 to $9 \mu \mathrm{m}$ range. In ionized PAHs, on the other hand, the situation is completely reversed. Enhanced by an order of magnitude relative to their neutral counterparts, the 6 to $9 \mu \mathrm{m} \mathrm{CC}$ stretching and $\mathrm{CH}$ in-plane bending modes now dominate the spectra of PAH cations. Conversely, suppressed by an order of magnitude, the $\mathrm{CH}$ stretching features have all but disappeared from the cation spectra. The $\mathrm{CH}$ out-of-plane bending modes are also suppressed in the cations, but much more modestly so $(\approx 2 x)$. Thus, as illustrated in Figure 3, ionization produces a global pattern of band intensities that is in much better agreement with the pattern of intensities observed in the interstellar emission spectrum than is the case for neutral PAHs. This relieves what has, since the time of its inception, been one of the most troubling qualitative difficulties of the interstellar PAH model - the disparity between the global appearance of the interstellar emission spectra and that of the initially available laboratory spectra of neutral PAHs.[97]

Within the framework of the PAH model, the interstellar spectrum arises from the combined emission of a complex mixture of PAHs. Therefore, to have any hope of reproducing the appearance of this spectrum, one must consider not the spectrum of any

one PAH, but the composite spectrum of a variety of different PAHs. Previous comparisons between the interstellar features and laboratory spectra have been forced to 


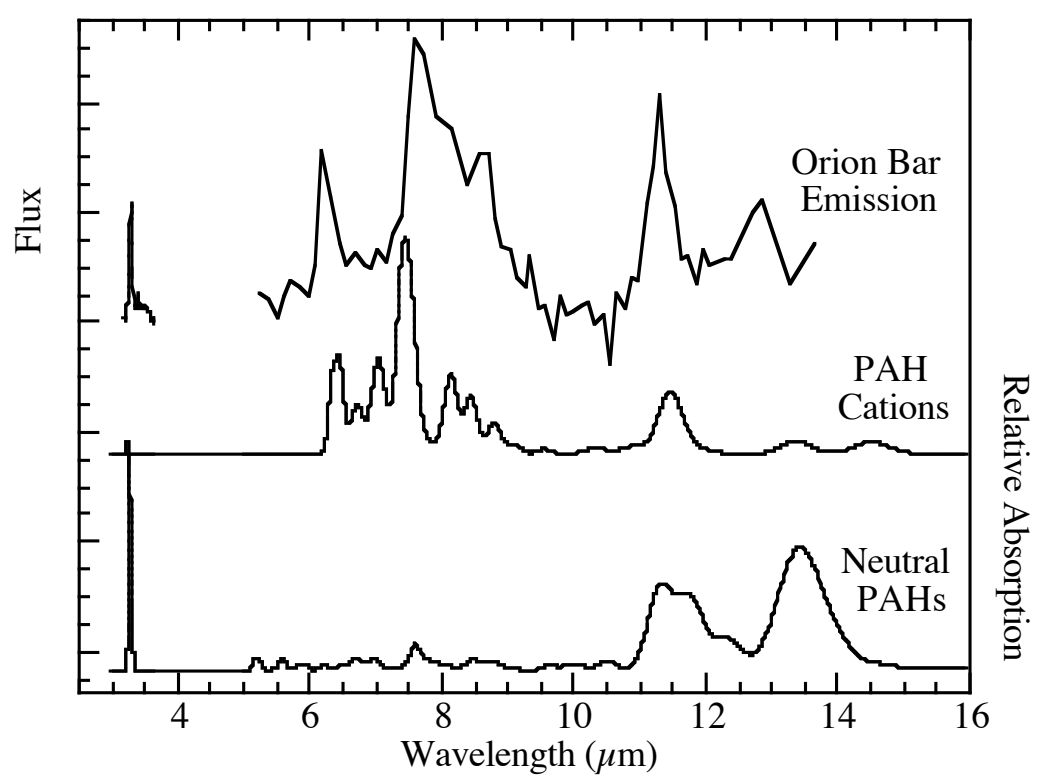

Figure 3. The absorption spectrum produced by coadding the spectra of the PAHs anthracene, tetracene, 1,2-benzanthracene, chrysene, pyrene, and coronene in their (a) neutral and (b) ionized forms compared to the emission spectrum of the ionization ridge in the Orion Nebula. This comparison shows that, for PAHs, ionization has a much greater influence on relative intensities than on peak frequencies, and that ionized PAHs are better candidates for the emission band carriers.

rely on the spectra of a single PAH or on limited combinations of a very few PAHs. With the advent of an extensive database of astrophysically relevant PAH spectra, comes the potential for much more comprehensive modeling of the emitting PAH population. This is illustrated in Figure 4 which shows a comparison between the emission from the ionized ridge in the Orion Nebula and the composite absorption spectrum generated by coaddition of $11 \mathrm{PAH}$ spectra, judiciously chosen from the laboratory database (See [97] for a discussion of the fitting procedure). Note that since the expected matrix shift $\left(5-10 \mathrm{~cm}^{-1}\right)$ and vibrational excitation red-shift between absorption and emission band positions $\left(5-15 \mathrm{~cm}^{-1},[52]\right)$ are significantly smaller than the $30 \mathrm{~cm}^{-1}$ natural linewidth expected from the interstellar emitters, these effects have not been compensated for in the figure.

\subsection{INTERSTELLAR PAHS: PROBING THE EVOLUTION OF INTERSTELLAR CARBON}

The following examples, adapted from [97], serve to illustrate how an analysis of the interstellar emission spectrum can yield important insight into the nature and properties of the PAH population, and how this information reflects the physical and chemical conditions within the emission regions themselves. 


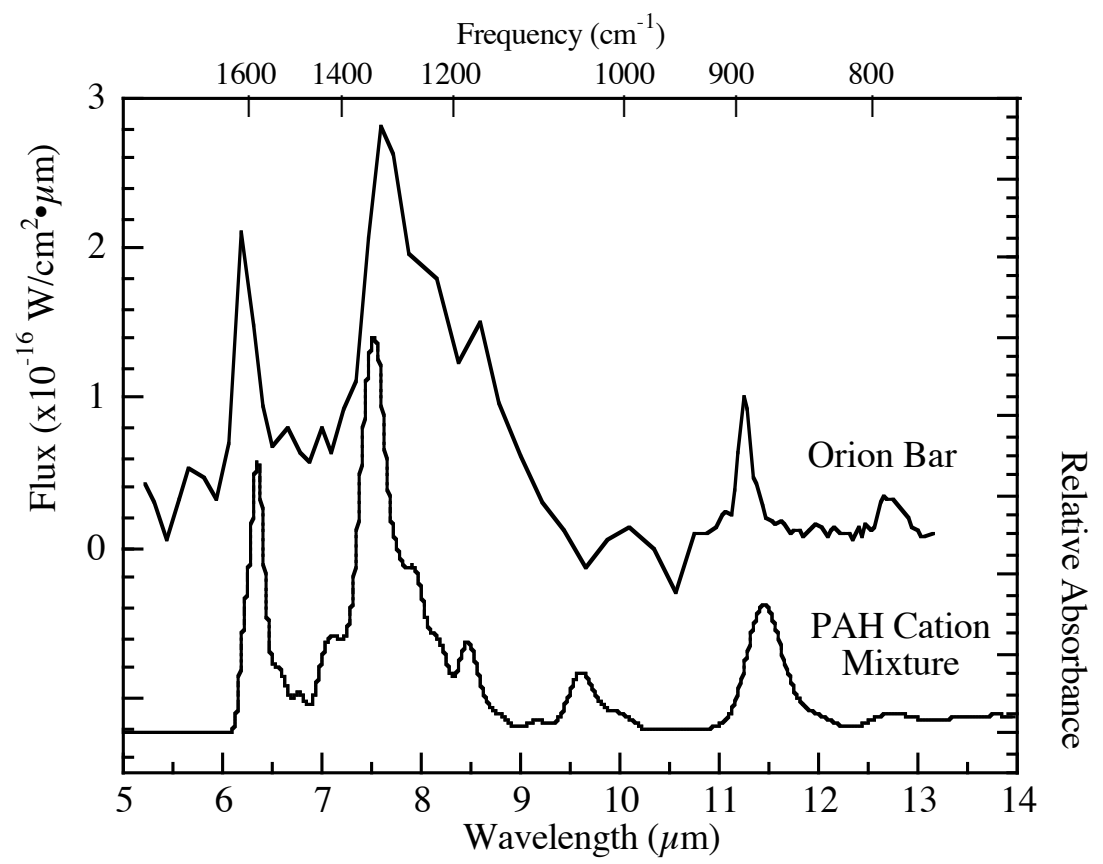

Figure 4. Comparison of the emission spectrum from the Orion ionization ridge to the composite absorption spectrum of a mixture composed only of ionized PAHs. The mixture consists of: $20 \%$ each benzo(k)fluoranthene ${ }^{+} ; 20 \%$ dicoronylene $^{+} ; 10 \%$ coronene $^{+} ; 10 \%$ benzo(b)fluoranthene ${ }^{+}$; $10 \%$ 9,10-dihydrobenzo(e)pyrene ${ }^{+} ; 10 \%$ phenanthrene $^{+} ; 5 \%$ benzo(ghi)perylene ${ }^{+} ; 5 \%$ tetracene ${ }^{+} ; 5 \%$ benz(a)anthracene ${ }^{+} ; 3 \%$ chrysene $^{+}$; and $2 \%$ fluoranthene $^{+}$. The Orion spectrum is reproduced from [98].

The protoplanetary nebula phase likely represents the earliest stage in the lifecycle of cosmic PAHs.[20,39,99-101] During the epoch of copious mass loss that punctuates the last stages of a star's life, $\mathrm{C}, \mathrm{N}$, and $\mathrm{O}$ produced during the final fitful stages of nucleosynthesis deep within the star are dredged up and cast off together with the majority of the dying star's atmosphere. If the abundance of carbon exceeds that of oxygen in this shell, a rich variety of carbon-rich compounds are formed. IRAS $22272+5435$, whose spectrum is shown in Figure 5, is a carbon-rich object undergoing just such a transformation.[102,103] The observed infrared emission is excited by the remaining, relatively cool (T 5,300 K, [103]), central giant star. Eventually, the outer layers of the star will be thrown off, exposing the ejecta to the harsh ionizing radiation of the still-extremely-hot ( $\mathrm{T} \sim 50,000$ to $150,000 \mathrm{~K}$ ), stellar core and ushering in the planetary nebular phase. Thus, this transition phase, which lasts on the order of $10^{3}$ years, is sometimes referred to as the proto-planetary nebula phase. The best fit we have found to the spectrum of IRAS $22272+5435$ using the ca. 1999 database is also shown in Figure 5. Inspection of the composition of this mixture (given in the figure 


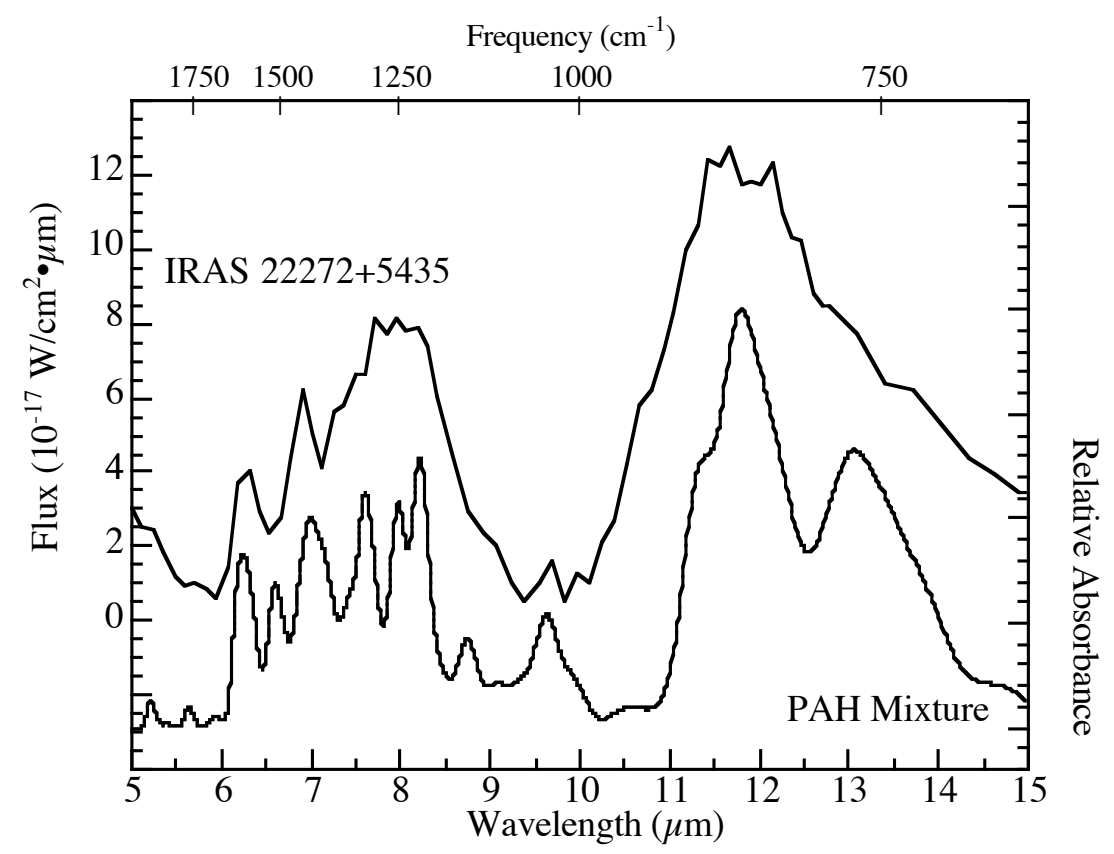

Figure 5. The emission spectrum from the protoplanetary nebula IRAS $22272+5435$ compared to the absorption spectrum produced from a mixture of neutral and cationic PAHs. The mixture is comprised of ("O" indicates a neutral species; " $"$ " indicates a cation): $18 \%$ dicoronylene ${ }^{\mathrm{O}}, 14 \%$ each naphthalene ${ }^{+}$and 9,10-dihydrobenzo(e)pyrene ${ }^{+}, 11 \%$ 9,10-dihydrobenzo[e]pyrene ${ }^{\mathrm{O}}, 10 \%$ each benzo[j]fluoranthene ${ }^{\mathrm{O}}$ and coronene $^{\mathrm{O}}$, and $3 \%$ each benzo[a]fluoranthene ${ }^{+}$, benzo[j]fluoranthene ${ }^{+}, \quad$ coronen $^{+}$, hexabenzocoronene ${ }^{+}, \quad$ dicoronylene $^{+}$, benzo[a]fluoranthene $e^{\mathrm{o}}$, naphthalene ${ }^{\mathrm{o}}$, and hexabenzocoronene ${ }^{\mathrm{o}}$. The spectrum of IRAS 22272 +5435 is reproduced from [102].

caption) reveals that it is dominated by neutral PAHs ( $60 \%)$ and that it includes species with a broad range of stabilities, from large, condensed PAHs (e.g. dicoronylene) to naphthalene, the smallest PAH. Note also that the mixture is internally consistent in that the neutral and cationic forms of the same PAHs have been used to construct the fit (i.e there are no PAHs present in ionized form, but not neutral form, and vice-versa). A mixture such as this is certainly reasonable when one takes into consideration the nature of the object. Here, in the region where aromatic compounds are beginning to appear and before they have been exposed to the ferocious radiation field of the coming planetary nebula phase, it is logical to expect that the emitting material would contain a diverse mixture of species, representing a wide range of thermodynamic stabilities. Furthermore, given the relatively benign radiation field produced by a 5,300 $\mathrm{K}$ star, it is also expected that both neutral and ionized species should contribute to the emission. The PAH population which provides the fit shown in Figure 5 reflects exactly these characteristics.

Next, consider the very different environment represented by the ionization ridge in the Orion Nebula. The spectrum of that region, together with that of the best-fit 
mixture of species drawn from the database, was shown previously in Figure 4. The Orion ionization ridge represents the interface between a cold, dense molecular cloud and an $\mathrm{H}$ II region.[98] Here the material originally produced in late stellar outflows has been "aged" for perhaps a billion years. During this time the material has been passed back and forth many times between diffuse and dense cloud phases of the ISM, alternately bathed in the harsh galactic interstellar radiation field and frozen into icy grain mantles. Now this material is being exposed to the ionizing radiation from the adjacent hot young $\mathrm{O}$ stars which make up the Trapezium. The composition of the mixture that provides the best fit to the Orion spectrum is quite revealing about the nature of the PAH population there. Unlike the proto-planetary nebula, where a substantial contribution from neutral PAHs was required to achieve a satisfactory fit, a mixture composed entirely of cationic PAHs is indicated for this region. Furthermore, the role of less stable PAH structures (i.e. less condensed) in the mix is substantially reduced compared to the protoplanetary nebula case. Instead, PAHs having more highly condensed (and therefore more thermodynamically favored) structures dominate the emission. In fact, four of the thermodynamically most favored PAHs in the mixture contribute $60 \%$ of the match to Orion shown in Figure 4 . Closer inspection of the figure also shows that, although the model spectrum reproduces all the major peaks and relative intensities of the Orion spectrum reasonably well, the $1300 \mathrm{~cm}^{-1}$ feature is significantly narrower. This reflects the lack of the prominant $1280 \mathrm{~cm}^{-1}$ component [104] and the broad emission plateau underlying the 1613,1310 and $1160 \mathrm{~cm}^{-1}$ bands observed in the astronomical emission.[26,27] These deficiencies are likely attributable to one or more classes of PAHs that are, as yet, not represented in the database and to the lack of any contribution from carbonaceous grains. The PAH population reflected in the model spectrum from Figure 4 is again entirely consistent with what one would expect given the nature and history of this object. The molecules found in this region are those which have survived the interstellar gauntlet and the fierce radiation from the nearby $\mathrm{O} / \mathrm{B}$ stellar association. Lesser stable components of the carbon-rich material initially ejected into the ISM have long since been 'weeded out' - either destroyed or isomerized into more stable structures by energetic processing. In addition, in the presence of the intense ultraviolet radiation from the nearby, young $\mathrm{O} / \mathrm{B}$ stars, it is expected that a substantial portion of the molecular population is likely to be ionized. Thus, it is entirely reasonable that we find the best-fit PAH mixture for the Orion ionization ridge reflects a disproportionately large contribution from the hardiest species and from ionized species.

While the PAH mixtures used to provide the spectral fits in the above examples are not unique, there is not a lot of variation possible in the choice of the dominant PAHs in each. Since IRAS $22272+5435$ and the Orion ionization ridge represent very different epochs in the evolution of cosmic carbon, the spectral differences reveal how carbonaceous material evolves as it passes from its circumstellar birth site into the general ISM. While there can be great variability in the appearance of the UIR spectrum between objects or from one region to another within one object [17], these differences can readily and naturally be accommodated by different PAH populations. The differences in the astronomical spectra are a direct consequence of differences in the composition of the emitting PAH population. The PAH population, in turn reflects a variety of physical and chemical conditions such as radiation field flux and energy, ionization states, carbon abundance, etc., in the emitting regions. Thus, given the ubiquity and intensity of the interstellar infrared emission features, PAHs hold the 
potential to provide a powerful probe of interstellar environments which span all the stages in the lifecycle of cosmic carbon.

Lastly, it should be emphasized that within the framework of the PAH model, the overall interstellar emission spectrum reflects contributions from both free, individual PAH molecules and from amorphous carbon grains comprised of a complex network of aromatic moieties. Earlier work has suggested that the particles can account for the broad, low contrast plateaus which underlie and vary with respect to the prominent sharp features.[26,48,105,106] Figures 3, 4, and 5 provide a compelling proof of concept that mixtures of free molecular PAHs and PAH ions can account for the discrete emission features. Indeed, considering the fact that while PAHs containing fewer than 50 carbon atoms likely account for $95 \%$ of the observed interstellar emission at $3.3 \mu \mathrm{m}$, they contribute only $\approx 30 \%$ of the emission at $6.2 \mu \mathrm{m}, 20 \%$ at $7.7 \mu \mathrm{m}$, and no more than a few percent at $11.2 \mu \mathrm{m}$ [96], it is remarkable that such good matches can be obtained using a database that samples only the very smallest end of the interstellar PAH population.

\subsection{INTERSTELLAR PAHS: PROBING THE INFRARED EMISSION ZONES}

As illustrated in the previous section, the ability of the laboratory spectra to accommodate the global appearance and variations of the interstellar spectrum supports the PAH hypothesis and provides a powerful new probe of a wide variety of interstellar conditions and histories. The most recent such applications have been aimed at achieving a fuller understanding of the PAH model and the nature of the interstellar infrared emitters in light of the latest spectroscopic observations provided by the ISO satellite. These applications are providing significantly deeper insight into the nature of interstellar PAHs and carbon's interstellar lifecycle. Two examples of this work will be presented and discussed briefly here. The first deals with the recent discovery of a discrete $16.4 \mu \mathrm{m}$ emission band and associated 15 to $20 \mu \mathrm{m}$ emission plateau and their interpretation within the context of the PAH model.[107,108] The second deals with a detailed analysis of the complex substructure of the interstellar emission spectrum from $10.5-14 \mu \mathrm{m}$, and the implications of this analysis regarding the size and structure of the emitting PAH population.[109]

2.5.1. The 15 to $20 \mu \mathrm{m}$ Emission Plateau and a New Emission Band at $16.4 \mu \mathrm{m}$ : Probing the presence of extremely large PAHs and PAH clusters. Within the framework of the PAH model, spectral features observed in the 15 to $30 \mu \mathrm{m}$ region are attributed to skeletal distortion modes of PAHs (i.e. in-plane and out-of-plane C-C-C bending). Given that these weak modes characteristically tend to congregate in this wavelength region, a broad, low-level continuum emission arising from the composite of many such bands was expected from PAH sources.[39] As shown in Figure 6, this expectation, initially based on a very limited set of PAH spectra but now supported by the full weight of our infrared database, is borne out by the latest ISO observations. This identification adds further credence to the PAH model and can be used to deduce specific characteristics of the interstellar PAH population in a given environment. Interestingly, the available PAH spectral data indicate that, despite the dramatic effect observed in other regions of the mid-infrared spectrum, ionization has little or no impact on the absolute intensities of the PAH bands in the $15-30 \mu \mathrm{m}$ range. Overall, the integrated intensities of the individual PAH bands observed in this region typically range 

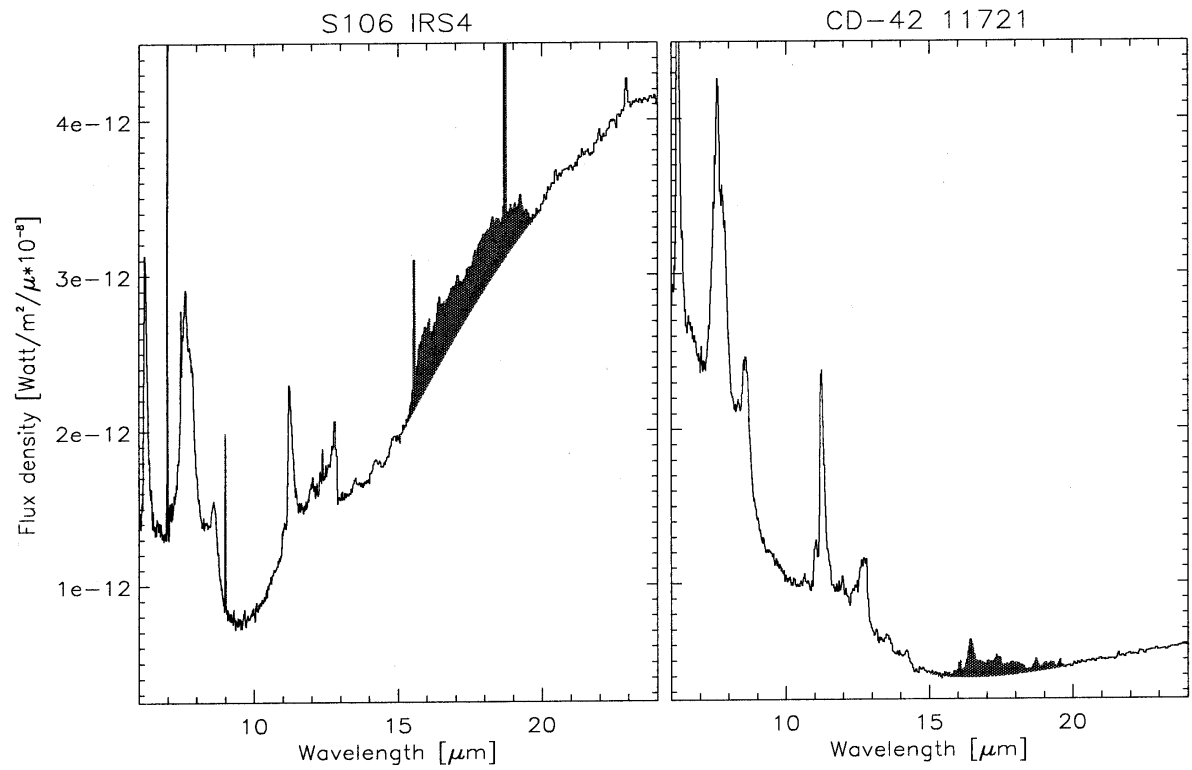

Figure 6. Two clear examples of the $15-20 \mu \mathrm{m}$ plateau emission discovered by ISO and attributed to the emission from a mixture of PAHs (shaded). Figure adapted from [107].

from 2 to $30 \mathrm{~km} / \mathrm{mol}$ independent of ionization state - roughly $5-10 \%$ that of the most intense bands in the spectrum (usually found between 11 and $14 \mu \mathrm{m}$ in the neutrals and between 7 and $8 \mu \mathrm{m}$ in the cations).[39,57,58,61-95] That notwithstanding, the sum of the intensities of the bands in the $15-30 \mu \mathrm{m}$ range can be comparable to that of the strongest band in the spectrum. This is consistent with the observed interstellar emission in which the total intensity under the $15-20 \mu \mathrm{m}$ plateau often exceeds that of the prominent interstellar $6.2 \mu \mathrm{m}$ emission feature.[107] Figure 7 shows a comparison between the average interstellar emission plateau and two composite model spectra based on the laboratory data. Details of the interstellar spectrum can be found in [107]. The model spectra were prepared by direct coaddition of astrophysically realistic simulations of the individual PAH spectra. Those simulations were prepared using the experimentally-measured band positions and intensities and assigning each band a nominal $30 \mathrm{~cm}^{-1}$ Gaussian profile consistent with the natural linewidth of the interstellar emitters. The figure clearly illustrates the broad feature produced by coadding the absorption spectra of just 7 matrix isolated PAHs. Note that not only is there good agreement between the interstellar and the laboratory data with respect to the intensity of the emission plateau compared to the rest of the spectrum, but also that there is good agreement as to the extent of the plateau (i.e. the wavelength range). This figure demonstrates that a complex of interwoven PAH bands in the 15-20 $\mu \mathrm{m}$ region can produce a structured quasi-continuum reminiscent of the observed plateau.

While there is some modest variation in the profile and extent of the emission plateau in different sources, the most pronounced distinction involves a relatively narrow, discrete band superimposed on the plateau and centered near $16.4 \mu \mathrm{m}$ (see Figure 7). While this feature is present in many of the regions displaying $15-20 \mu \mathrm{m}$ plateau emission, its intensity relative to that of the plateau is highly variable and there are regions where it is completely absent, suggesting a related but distinct origin. 


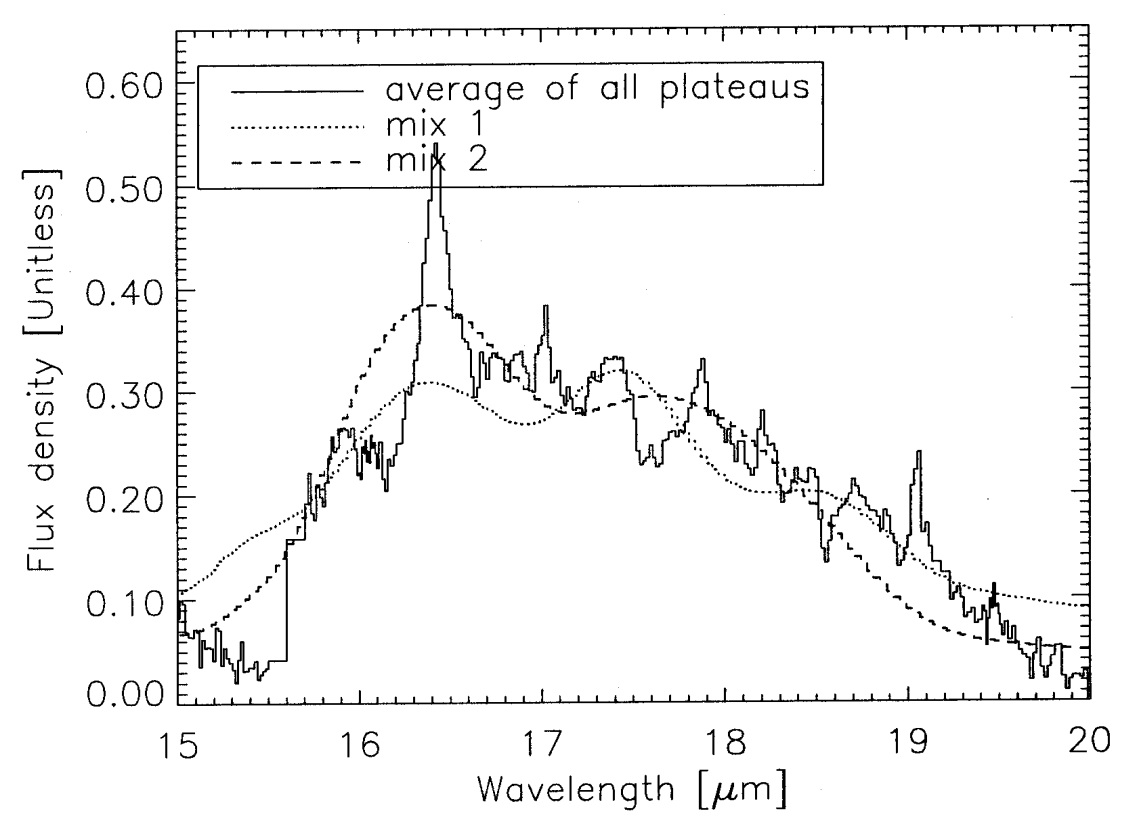

Figure 7. Comparison between the average $15-20 \mu \mathrm{m}$ interstellar emission plateau and laboratory spectra. The average emission was obtained by coadding all the interstellar plateaus normalized to the integrated intensity. The first laboratory spectrum (mix 1 ) is produced by the coadded spectra of anthracene (33\%), 1,2-benz[a]anthracene (33\%), and pentacene (33\%). The second laboratory spectrum ( $\mathrm{mix} 2$ ) is a spectrum produced by the coadded spectra of benzo(k)fluoranthene $(20 \%)$, pentacene $(40 \%)$, anthracene $(20 \%)$, and 1,2 benzanthracene $(20 \%)$. Figure adapted from [107].

Another singular aspect of this band is its FWHH - $\leq 10 \mathrm{~cm}^{-1}$ - substantially narrower then any other member of the emission band family and that expected based on our understanding of the photophysics of the interstellar emission process discussed above. While this latter characteristic remains enigmatic, we are not prevented from seeking an interpretation of a discrete feature at this position within the context of the PAH model.

Infrared modes of PAHs over roughly the $15-17 \mu \mathrm{m}$ spectral range are characterized by in-plane C-C-C bending motions, whereas those falling longward of about $17 \mu \mathrm{m}$ are dominated by out-of-plane warping of the carbon skeleton.[110] Interestingly, one class of PAHs that consistently show a band between 16 and $17 \mu \mathrm{m}$ which could overlap to generate a striking feature at this location are the fluoranthenes - PAHs which incorporate a five-membered ring in their skeletal structure. $[66,67,108]$ Based on this trend, it is reasonable to posit that a vibrational motion peculiar to the five-membered ring might be responsible for the feature observed at this position. This hypothesis, however, is not borne out by a detailed analysis of the specific atomic displacements associated with these modes. Such an analysis indicates that the fluoranthene modes between 16 and $17 \mu \mathrm{m}$ arise not from a vibration of the five-membered ring, per se, but instead from an in-plane distortion of a "pendant" hexagonal ring in their structures (i.e. a benzenoid ring fused to the remaining molecule along a single face). This distortion is illustrated in Figure 8. It involves an in-phase oscillation of the two opposing $\mathrm{C}$ atoms 


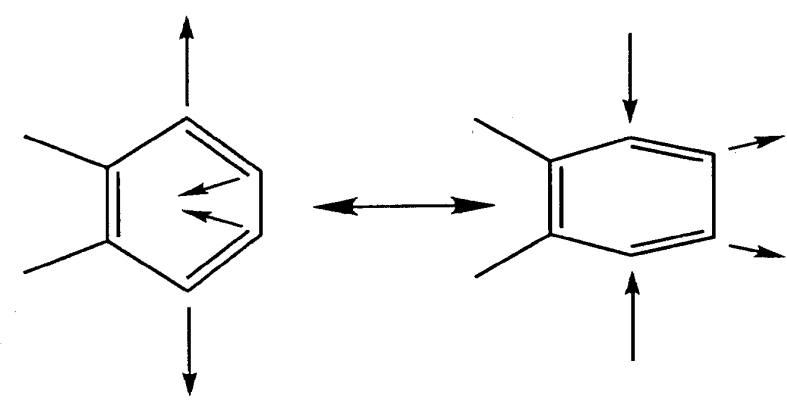

Figure 8. The in-plane $\mathrm{C}-\mathrm{C}-\mathrm{C}$ bending of the pendant ring that characterizes the vibrational modes between 16 and $17 \mu \mathrm{m}$ in the molecules in our database. It involves an in-phase oscillation of the two opposing $\mathrm{C}$ atoms immediately adjacent to the fused face of the pendant ring along a line parallel to that face. Figure adapted from [107].

immediately adjacent to the fused face of the pendant ring along a line parallel to that face. This characterization is confirmed by a similar analysis of other species from our database which carry a pendant benzenoid ring but no five-membered ring in their structures, and which exhibit a distinct feature near $16.4 \mu \mathrm{m}$. Thus, while it does not appear that the $16.4 \mu \mathrm{m}$ band specifically traces PAHs which carry five membered rings, it is nonetheless reasonable to suggest that the distinctive symmetry of the pentagonal ring represents one way to enhance the IR activity in C-C-C bending modes in the 16 to $17 \mu \mathrm{m}$ range which otherwise would be weak or forbidden. This effect may also become particularly important for asymmetric, non-condensed PAHs.

While this analysis does reveal the origin of the vibrational motion that produces a band in the 16 to $17 \mu \mathrm{m}$ region of the laboratory spectra, it should be noted that it is unlikely that the interstellar emission feature can be explained by this class of vibration alone. A pendant benzenoid ring will also necessarily contribute a quadruply-adjacent set of $\mathrm{CH}$ groups to the molecule. The out-of-plane bending mode of such a group typically falls near $14 \mu \mathrm{m}$ and is in all cases substantially stronger than the apical distortion band associated with that ring. Consequently, if the latter mode were at the heart of the interstellar $16.4 \mu \mathrm{m}$ band, there should be a significantly stronger emission feature near $14 \mu \mathrm{m}$. While the latest observations do reveal a band near this position, it is too weak to account for the implied quartet $\mathrm{CH}$ out-of-plane bending feature. Thus, while the pendant ring apical distortion identified here may contribute to the discrete $16.4 \mu \mathrm{m}$ interstellar emission band, it cannot, in and of itself, completely account for the feature.

Finally, the total integrated intensity of the $15-20 \mu \mathrm{m}$ emission plateau has been found to be as much as an order-of-magnitude higher in regions of massive star formation than in those regions where intermediate- and low-mass stars form. It has been suggested that this variation is reflective of an increase in the relative contribution from extremely large molecular PAHs and/or PAH clusters ( $z 500 \mathrm{C}$ atoms [107]).

Possessing large heat capacities (from a molecular standpoint), these species never attain the high $(\geq 1000 \mathrm{~K})$ peak temperatures achieved by the species which dominate the 3 $15 \mu \mathrm{m}$ emission (i.e. $\leq 50$ to $\sim 200 \mathrm{C}$ atoms). Consequently, they emit a disproportionate fraction of their energy at long wavelengths. Perhaps the prominence of this feature in regions of massive star formation reflects the effects of more extensive PAH coagulation compared to those regions which spawn intermediate and low-mass 
stars. Exploration of this possibility and its implications will require further observation and laboratory study.

2.5.2. PAH emission in the 10 to $15 \mu \mathrm{m}$ region: Probing the size and structure of the emitters. High resolution ISO/SWS observations in the $10-15 \mu \mathrm{m}$ spectral region for a wide variety of objects which exhibit the interstellar infrared emission bands has revealed a wealth of new structure and detail. This structure is illustrated in Figure 9 which shows the $10-15 \mu \mathrm{m}$ spectra of three representative sources: NGC 7027, CD-42 11721, and IRAS 18317-0757. Figure 9 reveals discrete features at 10.6, 11.0, 11.23, $12.0,12.7,13.5$, and $14.2 \mu \mathrm{m}$ with intensities relative to one another that are highly variable. A detailed discussion of the observations and spectral characteristics of the interstellar features in this region are provided in [109], and is not repeated here. Our purpose will be to consider what the spectral structure in this region reveals about the nature of the interstellar PAH population.

Chemists have long recognized the diagnostic value of the aromatic $\mathrm{CH}$ out-of-plane bending features in the 11 to $15 \mu \mathrm{m}$ spectral region for the classification of the aromatic ring edge structures present in a particular sample.[111] Specifically, the positions of the bands in this spectral region reflect the number of adjacent $\mathrm{CH}$ groups on the peripheral rings of the PAH structure.[20,54,97,111-115] Traditionally, aromatic rings carrying $\mathrm{CH}$ groups which have no neighboring $\mathrm{CH}$ groups (termed "non-adjacent" or "solo" CH groups) show IR activity between 11.1 and $11.6 \mu \mathrm{m}$. Likewise, activity between 11.6 and $12.5 \mu \mathrm{m}$ is indicative of two adjacent $\mathrm{CH}$ groups ("doubly-adjacent" or "duet" CH's) on the periphery of the PAH. Three adjacent $\mathrm{CH}$ groups ("triply-adjacent" or "trio" CH's) are indicated by activity in the 12.4 to $13.3 \mu \mathrm{m}$ region, and four adjacent $\mathrm{CH}$ groups ("quadruply-adjacent" or "quartet" $\mathrm{CH}$ 's) by activity between 13 and 13.6 $\mu \mathrm{m}$. Five adjacent $\mathrm{CH}$ groups ("quintuply-adjacent" or quintet $\mathrm{CH}$ 's) are indicated by features falling in the 13 to $13.7 \mu \mathrm{m}$ range. Trios and quintets also show a weak CCC bending mode in the $14-14.5$ range. Other such CCC bending modes were discussed above. Over the years the reliability of this region to yield insight into the molecular structure and ring sidegroup placement on aromatic samples has been verified again and again (see [112] and references therein). However, most of these chemist's guidelines were based on studies of small PAHs where varying patterns of sidegroup substitution were employed to achieve different degrees of $\mathrm{CH}$ adjacency. Furthermore, these chemist's 'rules-of-thumb' are based on spectroscopic studies of aromatic molecules in solution or solid mixtures which, as discussed in $\$ 2.2$, are of questionable astrophysical applicability.

From the standpoint of the astrophysical problem, there are two particularly pertinent issues regarding the characteristic PAH modes in the $10-15 \mu \mathrm{m}$ range that can be addressed by an analysis of the laboratory database. The first is the effect of ionization on the characteristic wavelength regions of the various $\mathrm{CH}$ adjacency classes. The second is the intrinsic integrated absorption strengths (A values) of the various adjacency classes and the effect of ionization on those strengths. Together, this information provides the tools not only to qualitatively infer the sorts of PAH edge structures present in the interstellar population, but also to quantitatively determine their relative amounts.

The first such analysis of the laboratory database was presented by Hudgins and Allamandola [112] and subsequently refined and extended in Hony et al.[109] The key points of those reports are summarized here in Figure 10 which schematically compares 


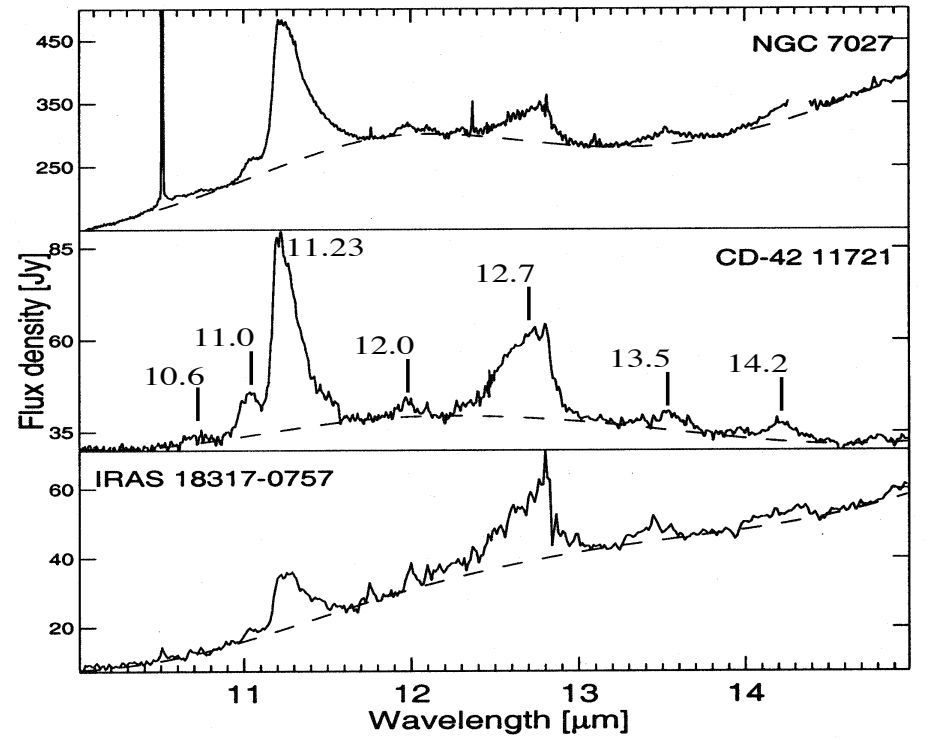

Figure 9. The $10-15 \mu \mathrm{m}$ spectra of three typical sources revealing an extremely rich collection of variable emission features with bands at 10.6, 11.0,11.23, 12.0,12.7, 13.5, and $14.2 \mu \mathrm{m}$. These features are perched on top of an emission plateau of variable strength, which extends across the entire region (dotted curves underlying spectra). Figure adapted from [109].

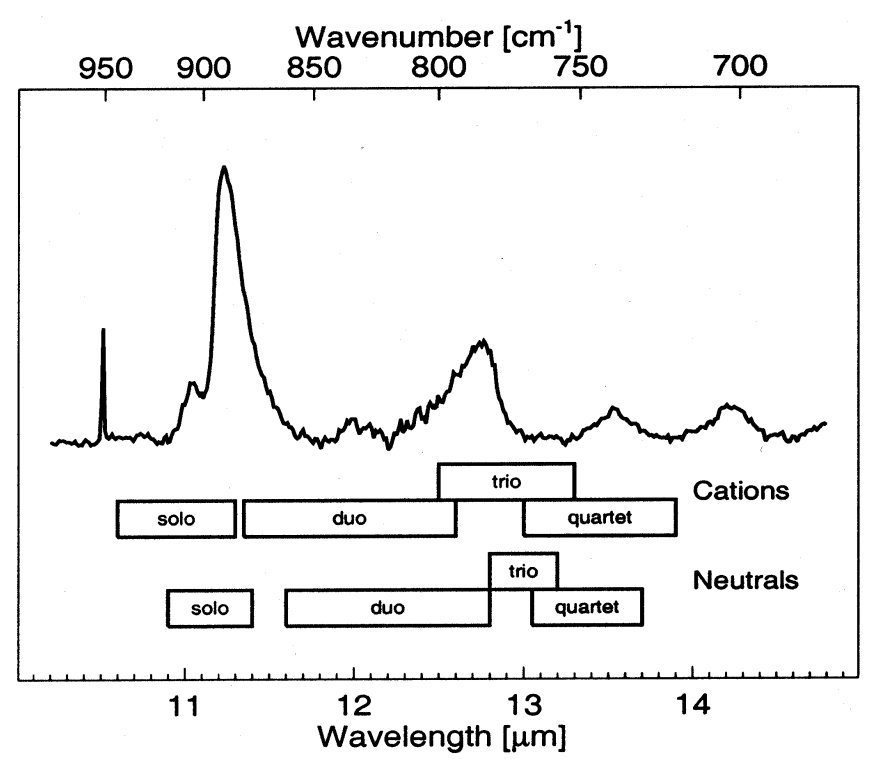

Figure 10. A comparison of the average interstellar spectrum (top) with the ranges characteristic of the out-of-plane bending modes of neutral and cationic PAHs (bottom). Details of the average interstellar spectrum are given in [109]. The boxes indicate the wavelength regions associated with the out-of-plane bending vibrations of the various adjacency classes of the peripheral $\mathrm{CH}$ groups as determined from matrix isolation spectroscopy. Figure adapted from [109]. 
the average interstellar emission spectrum with the wavelength regions associated with different $\mathrm{CH}$ adjacency classes for neutral and ionized, isolated PAHs. Perusal of this figure shows that, while the ranges for matrix-isolated neutral PAHs do not differ substantially from those reported in the literature (e.g. [111]), ionization produces some notable changes in region boundaries. Considering these modified domains and taking into account the roughly $0.1 \mu \mathrm{m}$ red- shift in the peak position for PAHs emitting at temperatures of $500-1000 \mathrm{~K}[49,50,52,60]$ we are left with the following conclusions:

- The broad, weak interstellar emission feature between 10.6 and $10.7 \mu \mathrm{m}$ and the stronger, distinct interstellar band peaking near $11.0 \mu \mathrm{m}$ fall in the region unambiguously attributable to PAH cations.

- The bulk of the $11.2 \mu \mathrm{m}$ interstellar band falls squarely within the region for the solo- $\mathrm{CH}$ modes of neutral PAHs and at the extreme long wavelength end of the range for solo cationic modes (note, however, that the neutral solo modes may extend out to 11.7).

- The domains indicated in Figure 10 show that regardless of the region definitions used, there can be little doubt that the weak interstellar $12 \mu \mathrm{m}$ band arises from duo modes.

- The blue shaded profile of the moderately strong band at $12.7 \mu \mathrm{m}$ corresponds most closely to the regions characteristic of the trio- $\mathrm{CH}$ modes of $\mathrm{PAH}$ ions and the duo$\mathrm{CH}$ modes of neutral PAHs. The trio modes of neutral species do not provide a good match.

- The weak $13.5 \mu \mathrm{m}$ feature falls squarely in the quartet domain for both the neutral and cationic species. However, this region also overlaps the lower of the two wavelength domains characteristic of quintet- $\mathrm{CH}$ groups, so a contribution from this class of $\mathrm{CH}$ group - however unlikely - cannot be ruled out.

Taken together, these conclusions regarding the edge-structural units make it possible to draw plausible average molecular structures for the PAHs which dominate the emission. This important insight is obtained through an analysis of the band intensities in the interstellar spectra rather than their positions. To this end, the absolute intensities of the solo-, duet-, trio-, and quartet-CH out-of-plane bending features as measured in the laboratory are presented in Table 3. Inspection of these data reveals that on a per- $\mathrm{CH}$ basis, the solo modes are 2 to 6 times more intense than the modes of the other adjacency classes. This is certainly one of the factors that contribute to the relative

Table 3. Average integrated intensities per $\mathrm{CH}$ group for the various classes of aromatic $\mathrm{CH}$ outof-plane bending modes arranged according to adjacency class.

\begin{tabular}{|c|c|c|c|c|c|c|}
\hline \multirow[t]{2}{*}{ Adjacency } & \multicolumn{2}{|c|}{$\langle A\rangle_{\text {neut. }}(\mathrm{km} / \mathrm{mol})$} & \multicolumn{2}{|c|}{$\langle A\rangle_{\text {cation }}(\mathrm{km} / \mathrm{mol})$} & \multicolumn{2}{|c|}{$\langle A\rangle_{\text {avg. }}(\mathrm{km} / \mathrm{mol})$} \\
\hline & per $C H^{a}$ & per group & $\operatorname{per} \mathrm{CH}^{\mathrm{c}}$ & per group & $\operatorname{per} C H^{a}$ & per group $a$ \\
\hline $\begin{array}{l}\text { Solo } \\
\text { duet }\end{array}$ & 25.7 & $\begin{array}{l}25.7 \\
8.8\end{array}$ & 24.1 & $\begin{array}{l}24.1 \\
7.4\end{array}$ & 24.8 & $\begin{array}{c}24.8 \\
8\end{array}$ \\
\hline $\begin{array}{c}\text { trio } \\
\text { quartet }\end{array}$ & $\begin{array}{l}10.1 \\
11.5\end{array}$ & $\begin{array}{l}30.1 \\
46.0\end{array}$ & $\begin{array}{c}9.0 \\
12.6\end{array}$ & $\begin{array}{l}27.0 \\
50.4\end{array}$ & $\begin{array}{c}9.6 \\
12.0\end{array}$ & $\begin{array}{l}28.8 \\
48.0\end{array}$ \\
\hline
\end{tabular}

$a 1$ solo group $=1 \mathrm{CH} ; 1$ duet group $=2 \mathrm{CH}$ 's; 1 trio group $=3 \mathrm{CH}$ 's; 1 quartet group $=4 \mathrm{CH}$ 's.

$b$ The average A value per $\mathrm{CH}$ group for the duet modes decreases rapidly with increasing molecular size settling down to a value near $4 \mathrm{~km} / \mathrm{mol}$ for PAHs larger than about $24 \mathrm{C}$ atoms. Therefore, this value is most appropriate to use for the purposes of constraining the edge structures of the large PAHs that dominate the emission at these wavelengths. 
prominence of the interstellar $11.2 \mu \mathrm{m}$ band. The data also show that, on average, ionization has little effect on the intensities of the $\mathrm{CH}$ out-of-plane bending features. Thus, for the purposes of analyzing band intensities in this region, it will be satisfactory to adopt a single average intensity for each of the adjacency classes, independent of ionization state.

To demonstrate the variability of the interstellar PAH population, we will focus on the extreme spectra of NGC 7027 and IRAS 18317-0757 - two objects whose emission spectra effectively bracket the range of observed relative band intensities. Furthermore, we will assume that the interstellar $11.2 \mu \mathrm{m}$ emission band arises from solo- $\mathrm{CH}$ modes, the $12.0 \mu \mathrm{m}$ band from duo modes, the $12.7 \mu \mathrm{m}$ from trios, and the $13.5 \mu \mathrm{m}$ from quartets. The salient data for the aforementioned objects is taken from [109] and summarized in Table 4.

Table 4. Intensities of the principal interstellar emission features in the $10-15 \mu \mathrm{m}$ region for two example objects.

\begin{tabular}{|c|cccc|ccc|}
\hline Object & $\mathrm{I}_{11.2^{\mathrm{a}}}$ & $\mathrm{I}_{12.0^{\mathrm{a}}}$ & $\mathrm{I}_{12.7^{\mathrm{a}}}$ & $\mathrm{I}_{13.5^{\mathrm{a}}}$ & $\mathrm{I}_{11.2^{/}} \mathrm{I}_{12.0}$ & $\mathrm{I}_{11.2} / \mathrm{I}_{12.7}$ & $\mathrm{I}_{11.2} / \mathrm{I}_{13.5}$ \\
\hline NGC 7027 & 142.7 & 6 & 35.9 & 9.7 & 23.8 & 4.0 & 14.7 \\
IRAS 18317-0757 & 10.5 & 1 & 15.6 & 2.0 & 10.5 & 0.67 & 5.3 \\
\hline
\end{tabular}

a intensities given in units of $10^{-14} \mathrm{~W} / \mathrm{m}^{2}$.

Taking the data from the last column of Table 3, we can calculate the ratio of the average intensity of the solo- $\mathrm{CH}$ out-of-plane bending features to that of each of the other adjacency classes. For example, the solo-to-duet intensity ratio is:

$$
\frac{\langle A\rangle_{\text {avg }}^{\text {solo }}}{\langle A\rangle_{\text {avg }}^{\text {duet }}}=\frac{24.8 \mathrm{~km} / \mathrm{mol}}{8 \mathrm{~km} / \mathrm{mol}}=3.1
$$

Similarly we find $\langle A\rangle_{\text {avg }}^{\text {solo }} \mid\langle A\rangle_{\text {avg }}^{\text {trio }}=0.86$ and $\langle A\rangle_{\text {avg }}^{\text {solo }} /\langle A\rangle_{\text {avg }}^{\text {quartet }}=0.52$. Now, these ratios can be used to calibrate the relative abundances of the various adjacency classes in the interstellar PAH population. This is accomplished by dividing the actual observed interstellar emission band ratios given in the right hand columns of Table 4 , by the corresponding intrinsic band intensity ratio:

$$
\frac{N_{\text {solo }}}{N_{x}}=\frac{\square I_{11.2} / I_{y} \square}{\square\langle A\rangle_{\text {solo }} /\langle A\rangle_{x} \square}
$$

where the subscript " $\mathrm{x}$ " indicates one of the multiply adjacent classes (duet, trio, or quartet) and the subscript " $y$ " indicates the position of the interstellar feature attributed to that class. For example, the ratio of the number of solo- to duet- $\mathrm{CH}$ groups in the emitting $\mathrm{PAH}$ population of NGC 7027 is:

$$
\frac{N_{\text {solo }}}{N_{\text {duet }}}=\frac{\left.I_{11.2} / I_{120}\right]}{\square A\rangle_{\text {solo }} /\langle A\rangle_{\text {duet }}}=\frac{23.8}{3.1}=7.7
$$

The ratios for the remaining adjacency classes for NGC 7027 and those of IRAS 183170757 have been calculated in similar fashion and are tabulated in Table 5.

Beyond simply quantifying the relative abundances of the various adjacency classes of peripheral $\mathrm{CH}$ bonds in the interstellar PAH population, these abundance ratios can further be used to construct example molecular structures that reflect the implied 
Table 5. The relative abundances of solo-, duet-, trio-, and quartet-CH groups in the emitting PAH population of two representative objects.

\begin{tabular}{|c|cc|}
\hline & NGC 7027 & IRAS 18317-0757 \\
\hline$N_{\text {solo }} / N_{\text {duet }}$ & 7.7 & 3.4 \\
$N_{\text {solo }} / N_{\text {trio }}$ & 4.7 & 0.78 \\
$N_{\text {solo }} / N_{\text {quartet }}$ & 28.2 & 10.2 \\
\hline
\end{tabular}

characteristics of the PAHs that dominate the $10-15 \mu \mathrm{m}$ interstellar emission in each object. Indeed, although the $\mathrm{CH}$ groups directly reflect only the edge structures of the emitting PAHs, these ratios indirectly constrain the minimum size of the dominant emitters. This is illustrated in Figure 11 which shows a series of example molecular structures that satisfy the abundance ratios of Table 5. In the figure, each structure is given along with its overall $\mathrm{C} / \mathrm{H}$ ratio, its solo-/duet- $\mathrm{CH}$ group ratio, and its solo-/trio$\mathrm{CH}$ group ratio. In general, solo- $\mathrm{CH}$ groups are indicative of long straight molecular edges, while duets and trios reflect corners. Quartets likely indicate pendant rings attached to such structures and the addition of one such ring for every few molecules in the interstellar population would be sufficient to account for their implied low relative abundance. In examining these structures, one should bear in mind that they represent just a few of many structures one might draw to satisfy the interstellar intensity ratios of Table 5. However, significantly, it is not possible to satisfy the interstellar intensity ratios with much smaller PAHs. Thus, to reproduce the dominance of the $11.2 \mu \mathrm{m}$ feature and the relative intensities of the bands at 12.0, 12.7, and $13.5 \mu \mathrm{m}$, one is naturally driven towards rather large molecules with at least 100-200 carbon atoms. This is entirely in keeping with previous theoretical calculations of the molecular sizes of the PAH species which account for most of the emission in these features.[60]

Structure 1 in Figure 11 reflects the abundance ratios derived for NGC 7027 and illustrates the type of PAH structure which must dominate the $10-15 \mu \mathrm{m}$ emission in that object. Of course, as mentioned above, many other structures are possible which satisfy the observational constraints equally well. Nevertheless, all such structures would share a number of important characteristics with this one - namely, they would
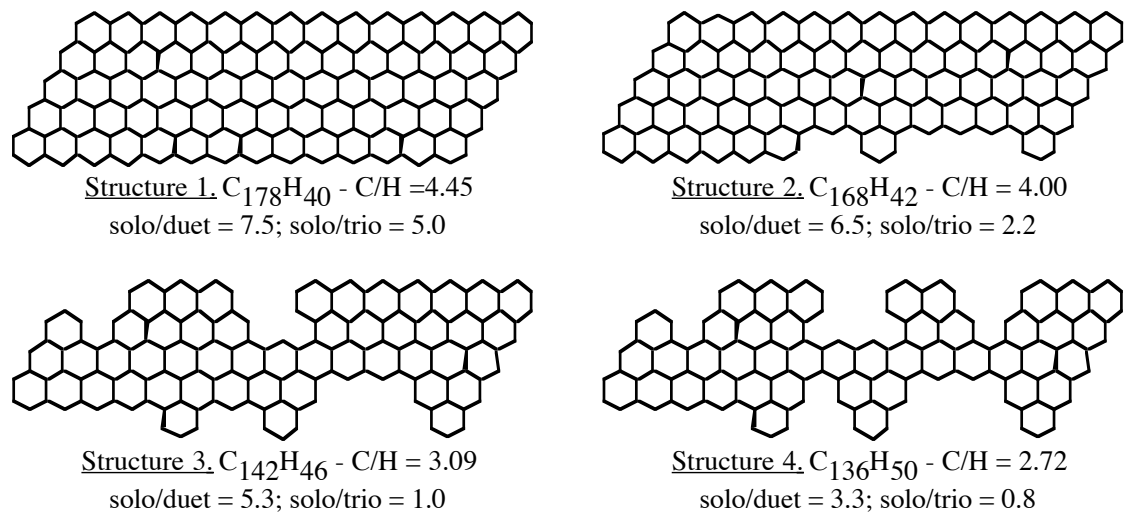

Figure 11. A series of example molecular structures that satisfy the structural constraints implied by the solo/duet and solo/trio abundance ratios given in Table 6 . The ratios of the number of solo$\mathrm{CH}$ groups to the number of duet- and trio- $\mathrm{CH}$ groups, as well as the overall $\mathrm{C} / \mathrm{H}$ ratio, is given below each structure. 
all possess reasonably condensed structures with long, straight edges and a minimum of corners, and only the occasional pendant ring thrown in for color. Thus, all of these structures would point to the same conclusion - that the PAH family in NGC 7027 is dominated by large compact PAHs.

The situation for IRAS 18317 , on the other hand, is very different. The observed ratios in that object imply a proportionally greater number of corners and/or uneven edges and a somewhat higher occurrence of pendant rings in the emitting population. Structure 4 in Figure 11 illustrates one way of achieving this. This effect could also be achieved by going to smaller, compact structures of the type shown in structure 1 (with shorter "flat" edges) or by breaking up structure 4 in two or more fragments. Structures 2 and 3 shown in Figure 11 represent intermediate cases that fall between the extremes of structures 1 and 4 . These have solo/duo and solo/trio ratios consistent with the relative interstellar band intensities of other objects which lie between the extremes of NGC 7027 and IRAS 18317-0757.[109] We surmise that the spectral evolution shown in Figure 9 belies a structural signature of the PAH population from large, highly condensed structures in some emission regions to open, uneven structures in others.

The nature of the molecular structures which dominate an emission region necessarily reflects the integrated history of the PAH family in that region. It is therefore interesting to note that the $10-15 \mu \mathrm{m}$ spectra of PNe typically exhibit the relatively strong $11.2 \mu \mathrm{m}$ (solo- $\mathrm{CH}$ ) band associated with large, highly condensed PAHs like structure 1 in Figure 11. As the principle site of PAH formation, the species found in late stellar ejecta have formed within the last few thousand years - but a moment by interstellar standards. Because "bay regions" - cavities in the edge structure of a PAH (c.f. Figure 11, structures 2 - 4) - are particularly susceptible to the addition of carbon atoms, it is not surprising that such edge structures should quickly be filled-in in regions of active PAH growth. In addition, the ferocious radiation field and dynamic processes characteristic of $\mathrm{PNe}$ would tend to weed out all but the most stable PAH structures. Both of these factors would be expected to drive the population toward more highly condensed structures, consistent with our analysis of the ISO spectra of these objects.

In contrast, HII regions (as exemplified by IRAS 18317-0757) tend to exhibit relatively strong $12.7 \mu \mathrm{m}$ (trio- $\mathrm{CH}$ ) bands. These are regions where hot, young $\mathrm{O} / \mathrm{B}$ stars illuminate material which has been processed for perhaps millions of years through both dense- and diffuse-cloud phases of the ISM. This processing involves both ablation and fragmentation by shocks and energetic particle bombardment, as well as chemical degradation such as oxidation and reduction by energetic processing in icy interstellar grain mantles as discussed in the following sections. As illustrated in structures 2 - 4 in Figure 11, these processes would be expected to gradually but inexorably eat away at the structure, reducing the overall level of ring condensation in the PAH population. Once again, this is consistent with our analysis of the ISO data.

\subsection{INTERSTELLAR PAHS: A SUMMARY}

The I/S PAH model has now moved beyond merely seeking to verify the presence of PAHs in space to the active exploitation of these ubiquitous species as probes of the ISM. The latest high-quality astronomical observations, supported by an everincreasing database of astrophysically relevant laboratory spectra of PAHs and PAH 
ions, is now providing insight into the conditions in IR-emitting regions at an unprecedented level of detail. Clearly, PAHs hold the potential to be an invaluable probe of the ISM, much as CO has been for the last quarter-century.

Given their demonstrated widespread abundance in molecular clouds, it is natural to wonder how PAHs might interact the other ubiquitous dust component of these regions - icy grains. In addition, since the members of the extended PAH family (small and large molecules, clusters, and particles) represent the single largest repository of organic material in our galaxy, it is natural to wonder what role these materials might play in the development of organically-based life. These questions and their implications are considered in the following sections.

\section{Interstellar/Precometary Ices: The Birthplace of Complex, Multifunctional Molecules}

As with the PAHs discussed in $\$ 2$, over the past 25 years infrared observations, combined with realistic laboratory simulations, have revolutionized our understanding of interstellar ices and dust, the feedstock of the Solar Nebula and the building blocks of comets. In this section we discuss the evidence for these ices. Although the dominant ice species are revealed by the IR spectra of dense molecular clouds, other species can also be important. These include species accreted directly from the gas phase and those produced within the ice itself by UV photon and high energy particle bombardment.

It is likely that the Solar Nebula incorporated material with a heritage in both the dense and diffuse ISM since there are many mixing cycles between these phases prior to star and planet formation.[116,117] It is thought that roughly $10 \%$ of dense cloud material becomes incorporated into stars and planets through each cycle, with the remainder dispersed back into the diffuse medium. While it is well beyond the scope of this paper to discuss the properties of each interstellar phase, detailed discussions of the general ISM can be found elsewhere.[4,5,6,7,118-121] In view of the astrobiological theme of this paper, we focus on the composition of IS ices, the birthplace and main reservoir of the extraterrestrial, complex, molecular material which ultimately seeded the planet with species that may have played a role in the evolution of life on earth.

\subsection{THE BUILDING BLOCKS OF INTERSTELLAR ICES}

Once a dense cloud is formed, thanks to the attenuation of the general IS radiation field, gas-phase and gas-grain chemistry leads to the production and sustaining of more complex species in the gas than possible in the diffuse ISM. At the same time, since the dust in a dense molecular cloud is so cold, $(\sim 20 \mathrm{~K})$, any polyatomic molecule striking a dust grain should condense [122], one might expect grain composition to reflect gas composition. However, as shown in Table 6, the simplest and most abundant gas-phase polyatomic molecules known from radio observations are much lower in abundance than their frozen counterparts. Thus, at this epoch, direct accretion of IS gas-phase species plays a very minor role in determining IS ice composition since the ice grains are chemical factories in their own right, generally harboring far greater amounts of material than the gas. 
Table 6. Comparison between the gas-phase and solid-state abundances for several molecular species normalized to hydrogen. This comparison shows that the interstellar ices contain the bulk of the simple interstellar polyatomic molecules.

\begin{tabular}{|c|c|c|c|c|}
\hline \multirow{2}{*}{ Molecule } & \multicolumn{2}{|c|}{ GAS PHASE $^{\mathrm{a}}$} & \multirow{2}{*}{$\begin{array}{c}\underline{\mathrm{ICE}}^{\mathrm{b}} \\
\text { NGC } 7538 \text { IR9 }\end{array}$} & \multirow{2}{*}{ Ice/Gas Ratio } \\
\hline & TMC-1 & OMC-1 & & \\
\hline $\mathrm{CO}$ & $8 \times 10^{-5}$ & $5 \times 10^{-5}$ & $6 \times 10^{-6}$ & 0.12 \\
\hline $\mathrm{H}_{2} \mathrm{O}$ & - & - & $6 \times 10^{-5}$ & $?$ \\
\hline $\mathrm{CH}_{3} \mathrm{OH}$ & $2 \times 10^{-9}$ & $1 \times 10^{-7}$ & $6 \times 10^{-6}$ & $60-3000$ \\
\hline $\mathrm{NH}_{3}$ & $2 \times 10^{-8}$ & - & $6 \times 10^{-6}$ & 300 \\
\hline $\mathrm{CO}_{2}$ & $5 \times 10^{-8}$ & - & $8 \times 10^{-6}$ & 160 \\
\hline $\mathrm{CH}_{4}$ & - & - & $6 \times 10^{-7}$ & $?$ \\
\hline
\end{tabular}

a Gas phase composition: TMC-1, [139]; OMC-1 [140]; $\mathrm{CO}_{2}$, [141]

b Ice mantle composition: [142]; $\mathrm{CO}_{2},[143]$; $\mathrm{CH}_{4}$, [144]

Throughout the cloud's lifetime, processes such as accretion of gas phase species, simultaneous reactions on the surfaces involving atoms, ions, and radicals, as well as energetic processing within the body of the ice by ultraviolet photons and cosmic rays all combine to determine the mantle composition.[3,4,118,123-138] These proceses are illustrated in Figure 12. Since hydrogen is 3 to 4 of orders of magnitude more abundant than the next most abundant reactive elements such as $\mathrm{C}, \mathrm{N}$, and $\mathrm{O}$ (Figure 1), overall grain surface chemistry is strongly moderated by the $\mathrm{H} / \mathrm{H}_{2}$ ratio in the gas. In regions where this ratio is large, $\mathrm{H}$ atom addition (hydrogenation) dominates and species such as $\mathrm{CH}_{4}, \mathrm{NH}_{3}$ and $\mathrm{H}_{2} \mathrm{O}$ are expected to be prominent.[125] If the $\mathrm{H} / \mathrm{H}_{2}$ ratio is substantially less than one, however, reactive heavy atoms such as $\mathrm{O}$ and $\mathrm{N}$ are free to interact with one another forming molecules such as $\mathrm{CO}, \mathrm{CO}_{2}, \mathrm{O}_{2}$ and $\mathrm{N}_{2}$. Thus, two qualitatively different types of ice mantle may be produced by grain surface reactions, one dominated by hydrogen-rich, polar molecules, capable of hydrogen-bonding and the other dominated by hydrogen-poor, non-polar (or only slightly polar), highly unsaturated molecules. Figure 12 also shows the first generation of products one might expect upon mantle photolysis (UV irradiation).

Interstellar ice compositions are revealed through their IR spectra. A star, fortuitously situated in or behind a molecular cloud, can provide a reasonably featureless, continuous mid-IR spectrum. As this radiation passes through the cold cloud, the intervening molecules in the gas and dust absorb at their characteristic vibrational frequencies. (This is quite distinct from the situation described earlier for the PAHs where it is the IR emission of vibrationally excited molecules that is measured.) Since the ISM between the "outside boundary" of the cloud and the Earth is far less dense, the IR absorption spectrum of objects obscured by molecular cloud material is mainly that of the dust in the dense molecular cloud. Since the ice features tend to dominates these spectra, interstellar ice composition can be analyzed by directly comparing the astronomical data with the spectra of ices prepared in a laboratory which duplicate the salient interstellar conditions. This can be accomplished using a cryogenic sample chamber similar to that used in the PAH studies described in $\$ 2$, except here thin layers of mixed molecular ices are deposited on the cold sample window. Sample ice thickness is comparable to that of all the interstellar grain mantles along the line of sight. In a typical experiment, the spectrum of the sample would be measured before and after several periods of exposure to UV radiation and thermal cycles. A more detailed discussion of this approach can be found elsewhere.[130,145,146] 


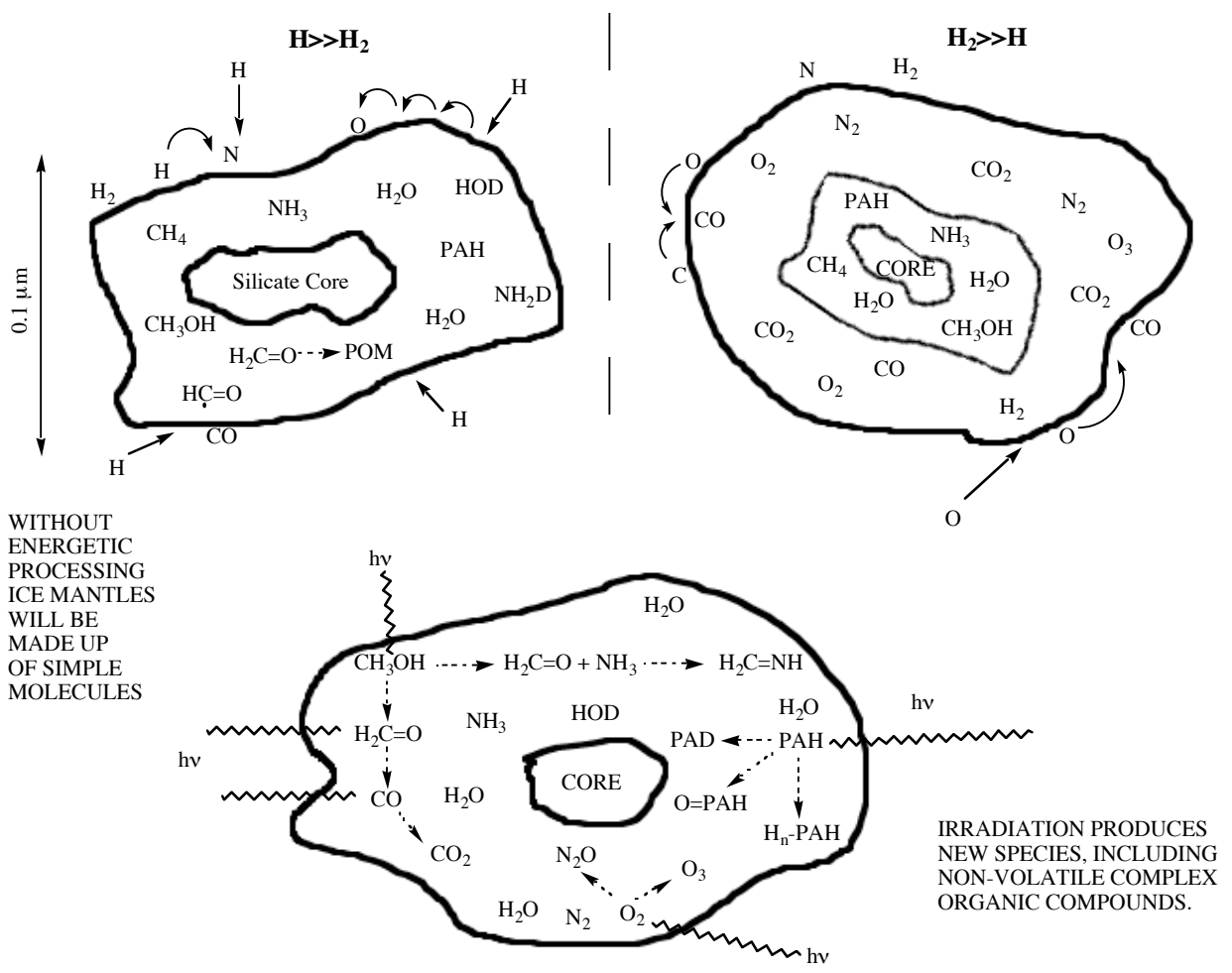

Figure 12. Schematic drawings of the types of ice mantles expected in dense molecular clouds. In regions where the $\mathrm{H} / \mathrm{H}_{2}$ ratio is much greater than one (top left), surface reactions tend to be reducing and favor the production of simple hydrides of the cosmically abundant $\mathrm{O}, \mathrm{C}$, and $\mathrm{N}$. In contrast, oxidized forms of these species are favored in regions where this ratio is much less than one (top right). Irradiation and thermal processing (bottom) of these ice mantles creates considerably more complex species and, ultimately, non-volatile residues.

Figure 13 shows a collage of comparisons between the spectrum of W33A, a protostar deeply embedded within a molecular cloud [147-149] with the laboratory spectra of interstellar ice analogs. With the exception of the strong absorption near $10 \mu \mathrm{m}$ for which the silicate $\mathrm{Si}-\mathrm{O}$ stretch overwhelms the overlapping ice features [146,150], all the absorptions in the spectrum of W33A are readily assigned to ice components. Excellent matches between the interstellar absorption features with laboratory spectra of the type shown in Figure 13 represent the basis of our knowledge of the composition of interstellar ice particles . Interestingly, until quite recently, much more was known of interstellar ice grain composition - particles hundreds of light years away - than of cometary ices in our own Solar System!

Over the past five years, deeper insight into the nature of interstellar ice and dust has been achieved through analysis of data from the Infrared Space Observatory (ISO). That mission has enabled measurement of the complete mid-IR spectrum with one instrument, eliminating the need to piece together bits from different telescopes taken under different conditions and with different spectral parameters. The IR spectra obtained with this 


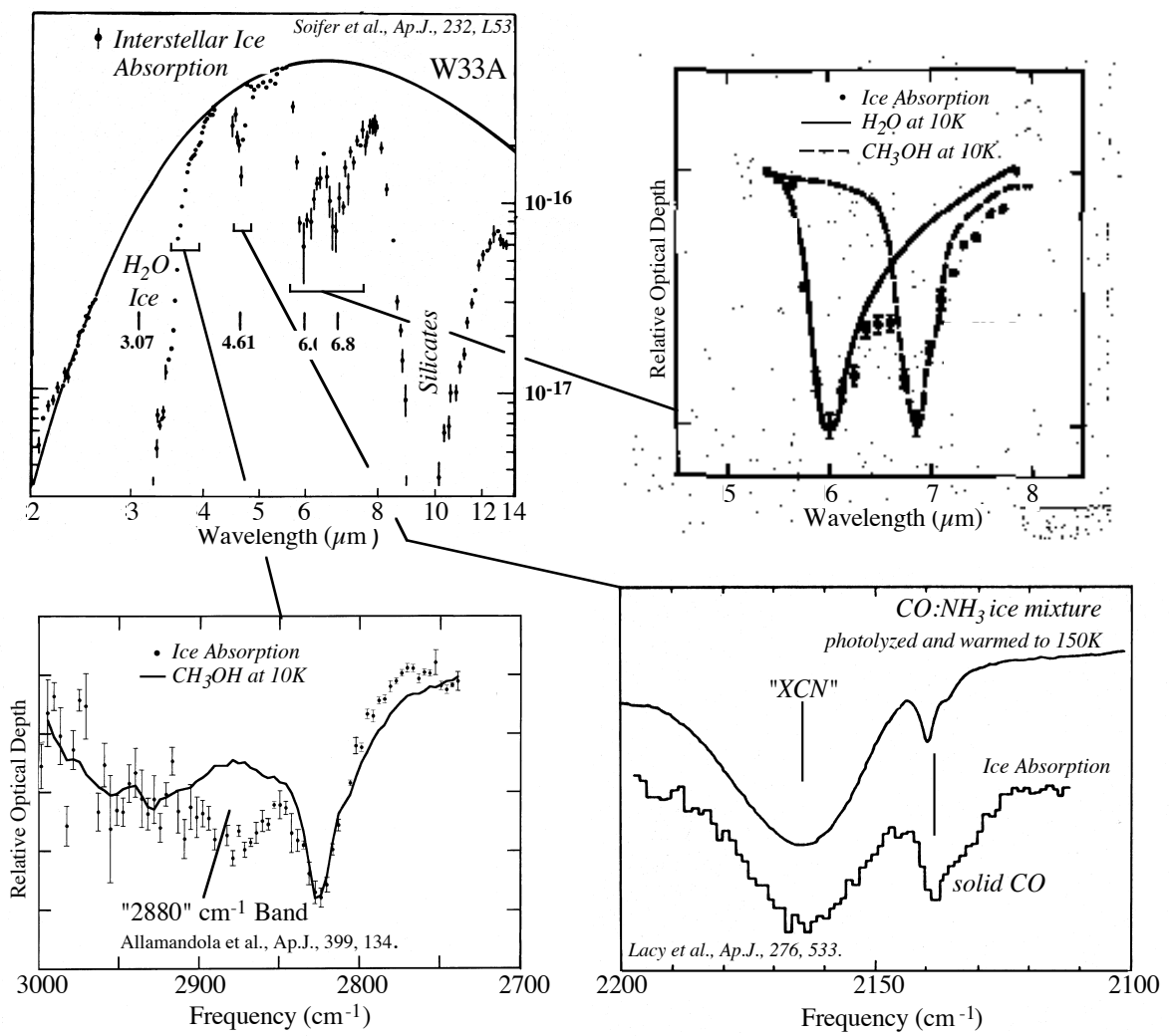

Figure 13. Comparisons of laboratory analog spectra with spectra from the object W33A, a protostar deeply embedded in a dense molecular cloud. Upper left: The dots trace out the interstellar spectrum and the solid line corresponds to the quasi-blackbody emission spectrum thought to be produced by the protostar. The strong absorption near $10 \mu \mathrm{m}$ is due to the silicate grains and the excess absorption labeled "2880 $\mathrm{cm}^{-1}$ Band" visible in the lower left-hand panel is thought to arise from interstellar microdiamonds in the cloud. All the remaining absorptions are produced by interstellar ices. These features are highlighted and compared to laboratory spectra on expanded scales in the surrounding frames. Lower left: The solid line is due to methanol in a laboratory ice. Lower right: The upper smooth line corresponds to a laboratory analog comprised of $\mathrm{CO}$ (sharp band) and $\mathrm{OCN}^{-}(\mathrm{XCN}$, broad band). Upper right: The solid and dashed lines correspond to spectra of $\mathrm{H}_{2} \mathrm{O}$ and $\mathrm{CH}_{3} \mathrm{OH}$ respectively.[142,147,149,151]

telescope has enabled this field to advance beyond analysis of the strongest spectral features, revealing important subtleties in the spectra that probe details of interstellar chemistry and grain evolution.[118,152-154] Table 7 lists the dominant interstellar ice constituents and gives their average abundance with respect to $\mathrm{H}_{2} \mathrm{O}$. A detailed discussion of this topic can be found elsewhere.[118] Here, only a very brief discussion of the most important components will be given in order to provide the basis for the subsequent discussion of more complex species. More information on each of these components can also be found in the literature.[155] 
Table 7. Composition and abundances of interstellar ice (relative to $\mathrm{H}_{2} \mathrm{O}$ ) compared to that deduced in Comets Halley, Hyakutake, and Hale-Bopp. The species listed above the dashed line in the second column have been definitely detected, those below have been tentatively identified. The evidence is good for all of these species. Cometary abundances from taken from:, [156] (Halley) and [157] (Hyakutake and Hale-Bopp). See text for interstellar ice references.

\begin{tabular}{|c|c|c|c|c|}
\hline \multirow{2}{*}{ Molecule } & Interstellar Ice & \multicolumn{3}{|c|}{$\underline{\text { Comet Parent Molecules }}$} \\
\hline & Abundance & Halley & Hyakutake & Hale-Bopp \\
\hline $\mathrm{H}_{2} \mathrm{O}$ & 100 & 100 & 100 & $\overline{100}$ \\
\hline $\mathrm{CO}_{(\text {polar ice })}$ & $1-10$ & & & \\
\hline $\mathrm{CO}_{\text {(non-polar ice) }}$ & $10-40$ & 17 & $6-30$ & 20 \\
\hline $\mathrm{CH}_{3} \mathrm{OH}$ & $<4-30$ & 1.25 & 2 & 2 \\
\hline $\mathrm{CO}_{2}$ & $1-10$ & 3.5 & $<7$ & 6 \\
\hline $\mathrm{XCN}$ & $1-10$ & --- & --- & --- \\
\hline $\mathrm{NH}_{3}$ & $5-10$ & 1.5 & 0.5 & 0.7 \\
\hline $\mathrm{CH}_{4}$ & $\sim 1$ & $<0.8$ & 0.7 & 0.6 \\
\hline $\mathrm{HCO}$ & $\sim 1$ & --- & --- & --- \\
\hline $\mathrm{H}_{2} \mathrm{CO}$ & $\overline{1}-\overline{4}$ & 3.8 & $0.2-1$ & 1 \\
\hline $\mathrm{H}_{2}$ & $\sim 1$ & --- & --- & --- \\
\hline $\mathrm{N}_{2}$ & $10-40$ & --- & -- & --- \\
\hline $\mathrm{O}_{2}$ & $10-40$ & --- & --- & --- \\
\hline $\mathrm{OCS}$ or $\mathrm{CO}_{3}$ & few & 0.2 & 0.1 & 0.3 \\
\hline $\mathrm{HCOOH}$ & low & --- & --- & --- \\
\hline
\end{tabular}

\subsection{1. $\mathrm{H}_{2} \mathrm{O}$ (water)}

$\mathrm{H}_{2} \mathrm{O}$ is the dominant ice component in dense clouds.[149,158-161] This identification is as secure as any based on infrared spectral matching since at least five interstellar features fit laboratory $\mathrm{H}_{2} \mathrm{O}$ ice spectra very well. Since the strong $3280 \mathrm{~cm}^{-1}(3.07 \mu \mathrm{m})$ band typically dominates the interstellar spectra (see Figure 13), it was one of the first features to be detected and identified in dense clouds. There is an extensive astronomical and laboratory literature dealing with this feature.[162-167]

\subsection{2. $\mathrm{CH}_{3} \mathrm{OH}$ (methanol)}

It was suggested early on that part of the prominent interstellar absorption near 1460 $\mathrm{cm}^{-1}(6.85 \mu \mathrm{m})$ shown in Figure 13 might be due to the $\mathrm{CH}$ deformation vibration of methanol.[149,168,169] However, a one band match in the IR is not sufficient for a firm identification in most cases and the unequivocal identification of methanol frozen on dust grains was not secure until the subsequent detection of other bands.[142,170173] However these bands were weaker than expected based on the depth of the 1460 $\mathrm{cm}^{-1}$ band, implying that other species contribute to the latter interstellar absorption. Aliphatic organic compounds and carbonates are reasonable candidates.[146] When present, methanol is often the second or third most abundant component of the ice after $\mathrm{H}_{2} \mathrm{O}$, with relative concentrations lying between about 5 and $30 \%$. The highest $\mathrm{CH}_{3} \mathrm{OH}$ ice abundances are seen toward these warmer regions.

\subsection{3. $\mathrm{CO}$ (carbon monoxide)}

After $\mathrm{H}_{2} \mathrm{O}$, carbon monoxide is the most studied interstellar ice component. $\mathrm{CO}$ has a characteristic absorption feature near $2140 \mathrm{~cm}^{-1}(4.67 \mu \mathrm{m})$, as shown in Figure 13. Its position, width, and profile are a sensitive function of the ice matrix in which it is 
frozen.[174-178] Many, but not all, of the lines-of-sight that contain $\mathrm{H}_{2} \mathrm{O}$ ice also contain $\mathrm{CO}$ ice and the relative strengths of the $\mathrm{H}_{2} \mathrm{O}$ and $\mathrm{CO}$ bands indicate $\mathrm{CO} / \mathrm{H}_{2} \mathrm{O}$ ratios ranging from 0.0 to as much as 0.3.[118,151,179-184] Although the $\mathrm{CO}$ band in a few objects has a position and profile consistent with $\mathrm{CO}$ frozen in $\mathrm{H}_{2} \mathrm{O}$-rich matrices, most lines-of-sight exhibit profiles indicative of $\mathrm{CO}$ frozen in non-polar matrices, i.e., ices thought to be dominated by molecules such as $\mathrm{CO}, \mathrm{CO}_{2}, \mathrm{O}_{2}$, and $\mathrm{N}_{2}$ rather than $\mathrm{H}_{2} \mathrm{O}$.[174,175,181,184,185] These are precisely the two sorts of mantles predicted on the basis of the $\mathrm{H} / \mathrm{H}_{2}$ ratio discussed earlier and sketched in Figure 12.

\subsection{4. $\mathrm{OCN}^{-}$(formerly XCN)}

The spectra of a limited number of lines-of-sight through dense clouds contain a broad, often weak, feature near $2165 \mathrm{~cm}^{-1}(4.62 \mu \mathrm{m})$ which was originally attributed to an unspecified nitrile (-CN) designated as XCN (Figure 13).[151,186,187] Although the number of examples is small, there is an indication that this feature is present only in the spectra of protostellar sources embedded within clouds and not in the quiescent regions sampled by background stars.[188-190] This suggests that the carrier of the feature is associated with the local environment of the embedded star. Laboratory work shows that the responsible species contains $\mathrm{O}, \mathrm{C}$, and $\mathrm{N}$; and that energetic processing is required for it's production. Although many laboratory studies have been carried out and excellent fits to the astronomical data have been realized, the precise molecular identity of the carrier remains elusive and contradictory conclusions abound.[186,191195] Nevertheless, the case in favor of an assignment to $\mathrm{OCN}^{-}$perturbed by the local ice mantle material is quite compelling.[192-194]

\subsection{5. $\mathrm{CO}_{2}$ (carbon dioxide)}

This species was long expected to be common in interstellar ices due to its ready production by energetic processing of interstellar ice analogs. However, due to screening by the its atmospheric absorptions, frozen interstellar carbon dioxide detection had to await a spaceborn spectrometer. The first detection of this species was made by d'hendecourt and Jourdain de Muizon using IRAS LRS data.[195] The sensitivity and spectral resolution limitations of this instrument however, permitted a serious study of only a few lines of site. This situation was vastly improved with the ISO SWS instrument. It is now known that frozen interstellar $\mathrm{CO}_{2}$ is similar in abundance to water ice, with the very strong CO stretch band near $2340 \mathrm{~cm}^{-1}$ often as deep as the 3400 $\mathrm{cm}^{-1}$ water ice band. The ISO spectra combined with extensive laboratory studies focusing on both the $\mathrm{CO}$ stretching and bending modes of this molecule have provided great insight into the various forms of mixed molecular ices present in dense clouds.[118,143,152,196-200]

\subsubsection{Other IS Ice Species}

In addition to the species considered specifically above, frozen $\mathrm{CH}_{4}$ (methane)[201,202] and $\mathrm{NH}_{3}$ (ammonia)[203] have also been clearly identified in interstellar ices. Furthermore, based on limited telescopic observations, laboratory studies of ice analogs, and theoretical chemistry models, a number of other molecular species are suspected of being present in interstellar ices in quantities on the order of a few percent relative to $\mathrm{H}_{2} \mathrm{O}$. These include $\mathrm{HCO}$ (formyl radical)[169]; $\mathrm{H}_{2} \mathrm{CO}$ (formaldehyde)[204]; $\mathrm{HCOOH}$, 
$\mathrm{HCOO}^{-}$, and $\mathrm{CH}_{3} \mathrm{COH}$ (formic acid, formate ion, and actetaldehyde, respectively)[153]; OCS (carbonyl sulfide)[205] or $\mathrm{CO}_{3}$ (carbon trioxide)[175]; and possibly ketones and/or aldehydes [169]. $\mathrm{N}_{2}$ and $\mathrm{O}_{2}$ might also be present as inferred by the profile of the $\mathrm{CO}$ band in certain lines-of-sight.[175] However, direct detection of these two important species in the IR will be very difficult, if possible at all.[118,199]

\subsection{PHOTOCHEMICAL EVOLUTION OF THE ORGANIC MATERIAL IN INTERSTELLAR/PRECOMETARY ICES AND THE PRODUCTION OF COMPLEX ORGANIC MOLECULES.}

The picture of mixed molecular interstellar ice described up to this point is supported by direct spectroscopic evidence (e.g. Figure 13). The identities, relative amounts and absolute abundances of the ice species listed in Table 7 and also discussed in [118] are sound. However, this is not thought to be the entire story. Indeed, from the origin of life perspective, this is only the beginning of the story. Here's why: the ices in dense molecular clouds are irradiated by UV photons and cosmic rays, breaking and rearranging chemical bonds within the ice to form new species. Although the abundance of this new material is only a few percent, this is a critically important process since it can create remarkably complex chemical groups and molecular species that cannot be made via gas phase and gas-grain reactions at the low temperatures and pressures characteristic of dense clouds. This is because the protection and ready availability of reaction partners in the solid phase favors chemical complexity and diversity while the energetics, radiation fields, and low densities of the gas-phase favor simplicity.

The interstellar/precometary ice composition in protostellar regions is also of particular relevance from the origin of life perspective since this is where new planets are formed. These are the regions in which the $\mathrm{OCN}^{-}$(formerly $\mathrm{XCN}$ ) and $\mathrm{CH}_{3} \mathrm{OH}$ bands are present, the tracers of in-situ energetic processing. The presence of methanol in these ices is of pivotal importance since it drives a rich interstellar ice photochemistry $[130,145,206]$ and plays an important role in gas phase chemistry.[207] Furthermore, methanol has profound effects on the physical behavior of $\mathrm{H}_{2} \mathrm{O}$-rich ices.[208] Since $\mathrm{CH}_{3} \mathrm{OH}$ is often an abundant ice component in comets (Table 7, cf. [209,210]), this may impact their structural [208] and vaporization [122] behavior as well.

Figure 14 shows the spectral evolution of an interstellar ice analog comprised of $\mathrm{H}_{2} \mathrm{O}: \mathrm{CH}_{3} \mathrm{OH}: \mathrm{CO}: \mathrm{NH}_{3}: \mathrm{C}_{3} \mathrm{H}_{6}(100: 50: 10: 10: 10)$ as a function of $\mathrm{UV}$ photolysis. Except for the $\mathrm{C}_{3} \mathrm{H}_{6}$, this analog mixture reflects the major interstellar ice components associated with protostellar environments. The exposure to UV results in the destruction of several species (particularly methanol) and the creation of others. The simplest and most abundant include $\mathrm{HCO}, \mathrm{H}_{2} \mathrm{CO}, \mathrm{CH}_{4}, \mathrm{CO}$, and $\mathrm{CO}_{2}$. As shown in Table 7, all of these new species have been identified in interstellar ices. The detection of these ice components in the dense molecular clouds does not necessarily imply radiation processing is responsible for their production since many of these molecules can also be formed by gas-phase or gas-grain chemistry. So, at present, all we can say is that observations are consistent with energetic processing. To reiterate, the strongest evidence that energetic processing is important, at least in some locations within dense clouds, is provided by the $\mathrm{OCN}^{-}$(formerly XCN) feature (e.g. Figure 13) and perhaps by 


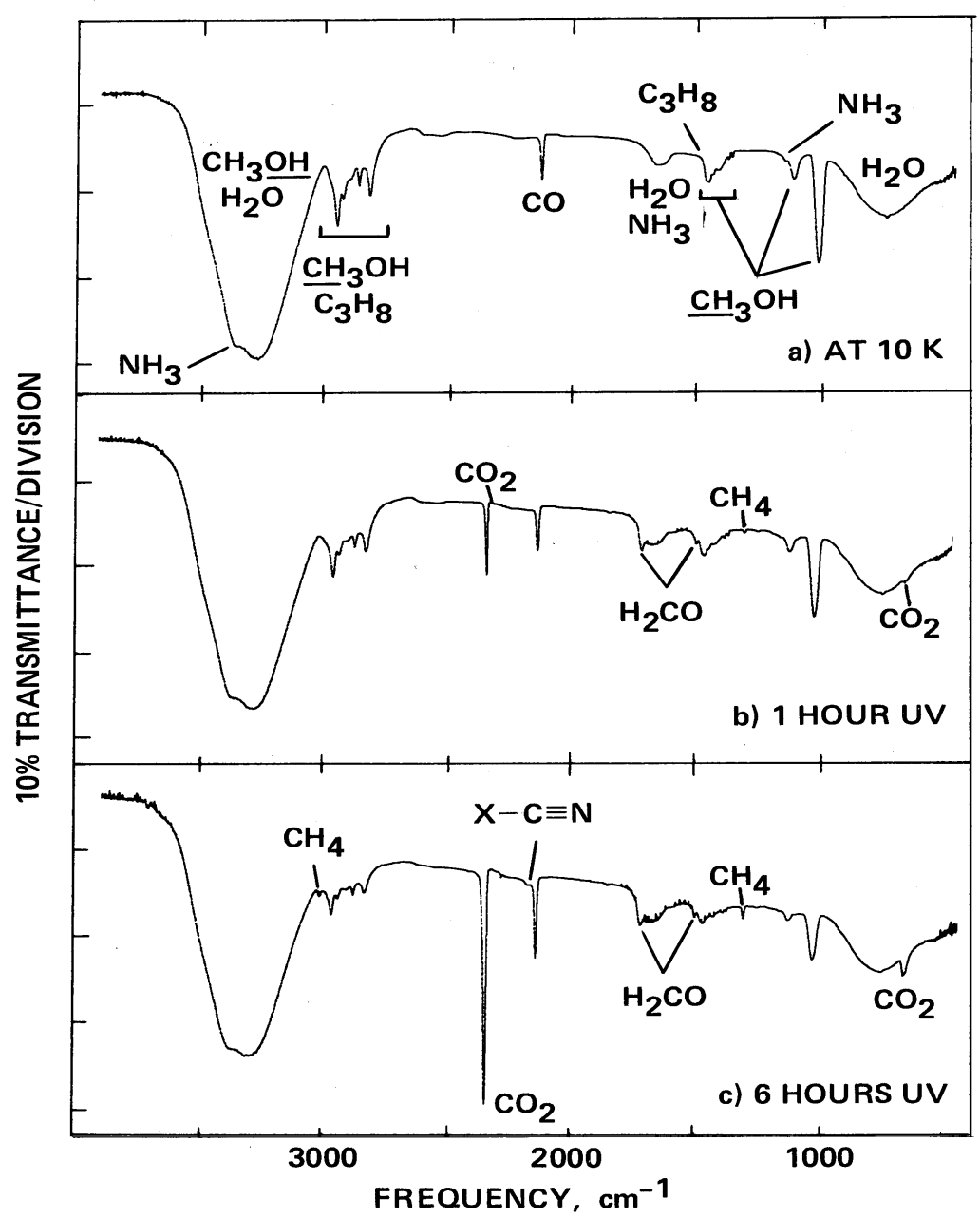

Figure 14. The photochemical evolution of an $\mathrm{H}_{2} \mathrm{O}: \mathrm{CH}_{3} \mathrm{OH}: \mathrm{NH}_{3}: \mathrm{CO}: \mathrm{C}_{3} \mathrm{H}_{8}$ (100:50:10:10:10) interstellar ice analog as traced by infrared spectra measured at $10 \mathrm{~K}$. The spectra were taken (a) before and after (b) 1 hour and (c) 6 hours of UV irradiation. Note the ready formation of $\mathrm{CO}_{2}, \mathrm{H}_{2} \mathrm{CO}, \mathrm{CH}_{4}$, and $\mathrm{XCN}$ at the expense of $\mathrm{CH}_{3} \mathrm{OH}$. Figure adapted from [145].

the ubiquity of $\mathrm{CO}_{2}$. The $\mathrm{OCN}^{-}$feature cannot be explained by any of the more abundant species predicted by gas and gas-grain chemical models, but is readily made by the radiative processing of laboratory ices containing $\mathrm{C}, \mathrm{N}$ and $\mathrm{O}$. Excellent, detailed descriptions of the UV induced chemical evolution of interstellar ice analogs can be found in the literature.[130,134,145,211-214] The role of methanol in the ice photochemistry discussed here is very critical to the kinds of compounds produced.

In contrast to photochemistry expected in the water -rich ices of dense star forming regions is that which characterizes the non-polar ices of quiescent regions. Here, since the polar species $\mathrm{H}_{2} \mathrm{O}, \mathrm{CH}_{3} \mathrm{OH}$, and $\mathrm{NH}_{3}$ aren't abundant, simple, low polarity species 
such as $\mathrm{CO}_{2}, \mathrm{~N}_{2} \mathrm{O}, \mathrm{O}_{3}, \mathrm{CO}_{3}, \mathrm{HCO}, \mathrm{H}_{2} \mathrm{CO}$, and possibly $\mathrm{NO}$ and $\mathrm{NO}_{2}$ are important photoproducts in laboratory simulations.[175] To date, ice composition in quiescent regions is not as well characterized as that in denser, high mass star forming regions.

For the remainder of this paper we will focus our attention on photochemical processing of the water-rich, polar ices associated with protostellar environments. The ice chemistry so far considered involves the photoproduction of the most abundant, simple species in the solid state at $10 \mathrm{~K}$. However, the full scope of the chemistry is much more complex and perhaps biologically relevant. Upon warm-up to about $200 \mathrm{~K}$ under vacuum one observes that the parent compounds and most volatile ice constituents sublime, leaving a residual mixture of less volatile species on the substrate. Of the staggering array of compounds produced from even the simplest starting ice containing $\mathrm{H}_{2} \mathrm{O}, \mathrm{CH}_{3} \mathrm{OH}, \mathrm{NH}_{3}$, and $\mathrm{CO}$, only a few have been identified. These are presented in Figure 15. In keeping with their expected low abundance, clear-cut spectroscopic evidence for these types of compounds in interstellar ices is presently lacking, although some of the weak spectral structure detected in the $2000-1250 \mathrm{~cm}^{-1}$ region is consistent with their presence. Additionally, spectral screening by the much more abundant, simpler ice species will likely represent an important, long-term obstacle. Higher quality astronomical spectra than those currently available will be needed to probe the species present at this level of concentration. Even then, identifications will likely be limited to chemical classes. Nonetheless, since many of these compound classes are of exobiological interest, a spectroscopic search for evidence of these materials in dense clouds would be of value.

Species evident at $200 \mathrm{~K}$

$$
\mathrm{CH}_{3} \mathrm{CH}_{2}-\mathrm{OH} \text { (ethanol) }
$$<smiles>NC=O</smiles><smiles>CC(N)=O</smiles>

Species remaining at $300 \mathrm{~K}$

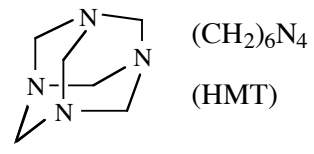

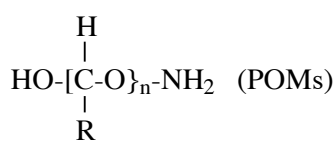

$\mathrm{CH}_{3} \mathrm{CH}_{2}-\mathrm{OH}$ (ethanol)<smiles>[R]C([R])=O</smiles><smiles>[R]C(N)=O</smiles>

Figure 15. Identified compounds produced by the $10 \mathrm{~K}$ UV photolysis and subsequent warm-up of the realistic interstellar ice analogs $\mathrm{H}_{2} \mathrm{O}: \mathrm{CH}_{3} \mathrm{OH}: \mathrm{NH}_{3}: \mathrm{CO}(100: 50: 10: 10)$ and $(100: 50: 1: 1)$. Figure adapted from [130]. 
The residue that remains on the window at room temperature is of particular interest from a prebiotic perspective. It is quite plausible that this type of material is closely related to that preserved in comets, meteorites and interplanetary dust particles (IDPs) and it is believed that these sources deliver between 12 and 30 tons of organic material to Earth monthly. During the period of great bombardment some 4 billion years ago, the amount of extraterrestrial organic material brought to the prebiotic early Earth was many orders of magnitude greater. Thus, this type of material could have been an important source of prebiotic organic matter on the early Earth and perhaps played an important role in the emergence of life.

Now, let's consider more closely the molecules of biogenic interest which are produced upon photolysis of realistic interstellar ice analogs. The word realistic is used to indicate that the laboratory ice composition reflects the interstellar polar ice composition shown in Table 7. The standard ice we study has a starting composition of $\mathrm{H}_{2} \mathrm{O}: \mathrm{CH}_{3} \mathrm{OH}: \mathrm{NH}_{3}: \mathrm{CO}(100: 50: 1: 1)$ or $\mathrm{H}_{2} \mathrm{O}: \mathrm{CH}_{3} \mathrm{OH}: \mathrm{NH}_{3}: \mathrm{CO}(100: 50: 5: 5)$. As evident from Table 7, these have higher methanol concentrations $\left(\mathrm{H}_{2} \mathrm{O} / \mathrm{CH}_{3} \mathrm{OH} \sim 2 / 1\right)$ than is currently considered $\left(\mathrm{H}_{2} \mathrm{O} / \mathrm{CH}_{3} \mathrm{OH} \sim 5 / 1\right)$ appropriate. There is, however, good reasons for this choice. It has been more than 15 years since we first undertook to study the photochemistry of interstellar mixed molecular ices. Since there was, at the time, no in-depth knowledge of the types of chemicals produced in this manner it was decided to focus our efforts on a consistent composition and at that time it was believed that the correct $\mathrm{H}_{2} \mathrm{O} / \mathrm{CH}_{3} \mathrm{OH}$ ratio was $2 / 1$. By the time the interstellar methanol concentration was called into question, a significant amount of work had already been undertaken and, a large body of knowledge and insight had already been gained into the chemistry of that ice, setting the stage for an attempt to analyze the minor photoproducts, a very challenging task. Moreover, experiments carried out at the lower methanol concentrations now thought to be more representative of interstellar ice composition have now been proven to yield essentially the same array of new compounds, albeit in significantly lower yields. As it is, even with the higher methanol concentrations, in order to produce sufficient material for one analytical run requires between two to three months of constant sample preparation. Thus, using a single apparatus for sample preparation it is typically possible to do only four to five experiments per year. Reducing the yield would shift this from a very difficult project to one that is impossible given our current analytic capabilities. Interestingly, rather recently, a $\mathrm{H}_{2} \mathrm{O} / \mathrm{CH}_{3} \mathrm{OH}$ ratio of $\sim 3 / 1$ has been reported [173], challenging the low methanol paradigm and more in line with the ice compositions considered here.

Lastly, a comment is in order regarding the other abundant interstellar ice components $\mathrm{CO}_{2}, \mathrm{XCN}, \mathrm{CH}_{4}, \mathrm{H}_{2} \mathrm{CO}$, and $\mathrm{HCO}$ listed in Table 7 . We are not concerned with their absence in the starting mixture because, as explained above (e.g. Figure 14, they are readily produced upon photolysis at concentrations consistent with the observations. As these are produced at the expense of methanol [145], lowering that species concentration, we believe that all the major interstellar ice ingredients known toward massive protostellar environments are represented in our experiment and at roughly the correct concentrations.

\subsubsection{Complex Organic Production in Ices without PAHs}

The residue produced from photolyzed methanol-containing ices which remains on the substrate at room temperature is rich in the cage molecule Hexamethylenetetramine 
(HMT, $\mathrm{C}_{6} \mathrm{H}_{12} \mathrm{~N}_{4}$ ).[130] This contrasts with the organic residues produced by irradiating mixed molecular ices which do not contain methanol [212,213,215] and those produced in thermally promoted polymerization-type reactions in unirradiated realistic ice mixtures.[216] In those experiments HMT is only a minor product in a residue dominated by a mixture of POM related species. (Interestingly, POMs already start to form in these ices at temperatures as low as $40 \mathrm{~K}$. Further, POM-like species have been suggested as an important organic component detected in the coma of Comet Halley.[217]) In addition to the possible prebiotic activity of HMT itself, its synthesis by photolysis implies the presence of several other biogenically interesting intermediates in these ices as well. For example, there are a variety of secondary carbon and nitrogen containing species (including amino acids) which are readily formed by HMT hydrolysis, thermalization, or photolysis, all processes which can occur during an interstellar ice grain, comet, or asteroid's lifetime. Since ultraviolet photolysis of HMT frozen in $\mathrm{H}_{2} \mathrm{O}$ ice produces the $\mathrm{OCN}^{-}$band [218], HMT may be related to the carrier of that band in dense clouds and, perhaps to the $\mathrm{CN}$ observed in cometary comae. These laboratory simulations of grain processing have also influenced our understanding of interstellar, gas phase chemistry. The HMT production pathway proposed in [130] involves the intermediate methylimine $\left(\mathrm{CH}_{2} \mathrm{~N}\right)$. Armed with this information, radioastronomers searched for and found methylimine to be widespread throughout the gas in many molecular clouds.[219] Beyond HMT and POMs, the involatile residue of photolyzed, methanol containing ices comprises lower concentrations of a bewildering array of organic compounds.

Due to the extreme complexity and analytical challenge posed by deeper analysis and encouraged by recent interest in the biological application of these results, effort has been redirected from solely establishing the chemical inventory of species produced in these interstellar/precometary ice analogs to searching for the presence of specific biogenically important species. This effort has required the use of two new techniques. These are High Performance Liquid Chromatography (HPLC) and laser desorption-laser ionization mass spectroscopy ( $\left.{ }^{2} \mathrm{MS}\right)$. Access to the latter technique has been made possible through a collaboration with Prof. R. Zare and his colleagues at Stanford University. While neither technique can provide an unequivocal identification directly, they are both particularly suited to microanalysis and give very valuable insight into the chemical properties of the compounds that make up the residue.

Figure 16 shows the HPLC chromatogram of one of the residues compared to a soluble extract from the primitive meteorite Murchison. There are two conclusions to be drawn from this figure. First, since each peak represents a different compound, or more likely a different family of compounds, both the laboratory residue and meteoritic extract are complex chemical mixtures. Second, the similarity in peak distributions between the two samples indicates that the kinds of chemicals present in each sample are similar. This similarity raises the interesting question, "Do the families of compounds in carbonaceous meteorites have an interstellar ice/cometary heritage?"

Figure 17 shows the $\mathrm{L}^{2} \mathrm{MS}$ of the residue from a photolyzed $\mathrm{H}_{2} \mathrm{O}: \mathrm{CH}_{3} \mathrm{OH}: \mathrm{NH}_{3}: \mathrm{CO}$ (100:50:1:1) ice. Mass spectra such as this provide us with further critical insight into the nature of the residue, showing that there are hundreds of compounds produced. Further, this shows that they are far more complex than the starting materials. The new materials produced are responsible for the envelope spanning the mass range from about $100 \mathrm{M} / \mathrm{Z}$ to $350 \mathrm{M} / \mathrm{Z}$. (M/Z is equivalent to an atomic mass unit). Given that none of 


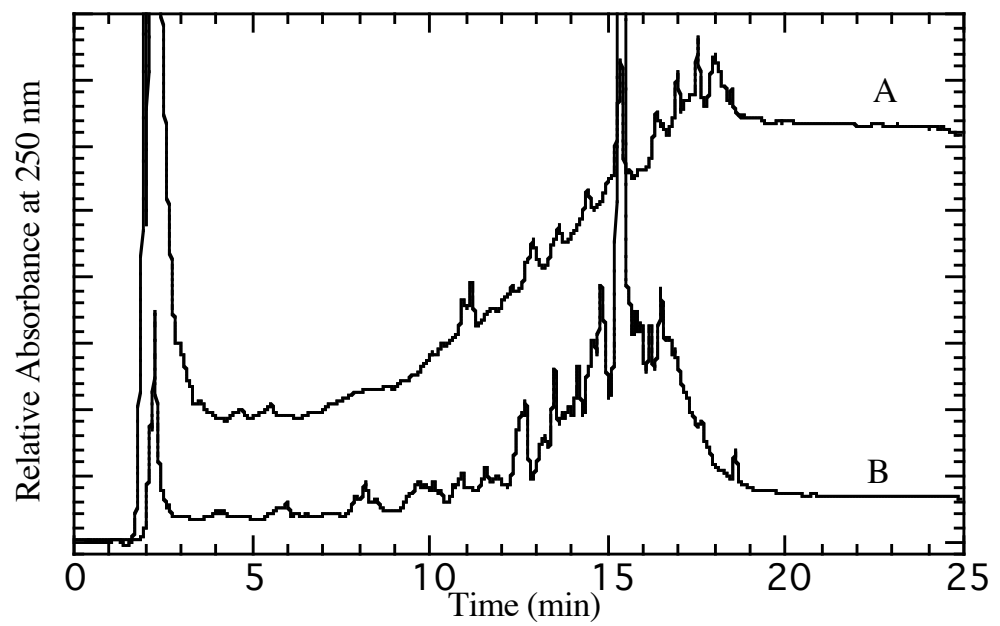

Figure 16. The high precision liquid chromatogram (HPLC) of (A) the room temperature residue produced by UV photolysis of an $\mathrm{H}_{2} \mathrm{O}: \mathrm{CH}_{3} \mathrm{OH}: \mathrm{NH}_{3}: \mathrm{CO}(100: 50: 1: 1)$ ice (profile magnified 10x) and (B) mixed acid and base extracts of Murchison meteorite. HPLC conditions: $1 \mathrm{~mL} / \mathrm{min} 50 \%$ water, $50 \%$ methanol to $100 \%$ methanol in 15 minutes, $\mathrm{C}_{18}$ reverse phase analytical column.[206]

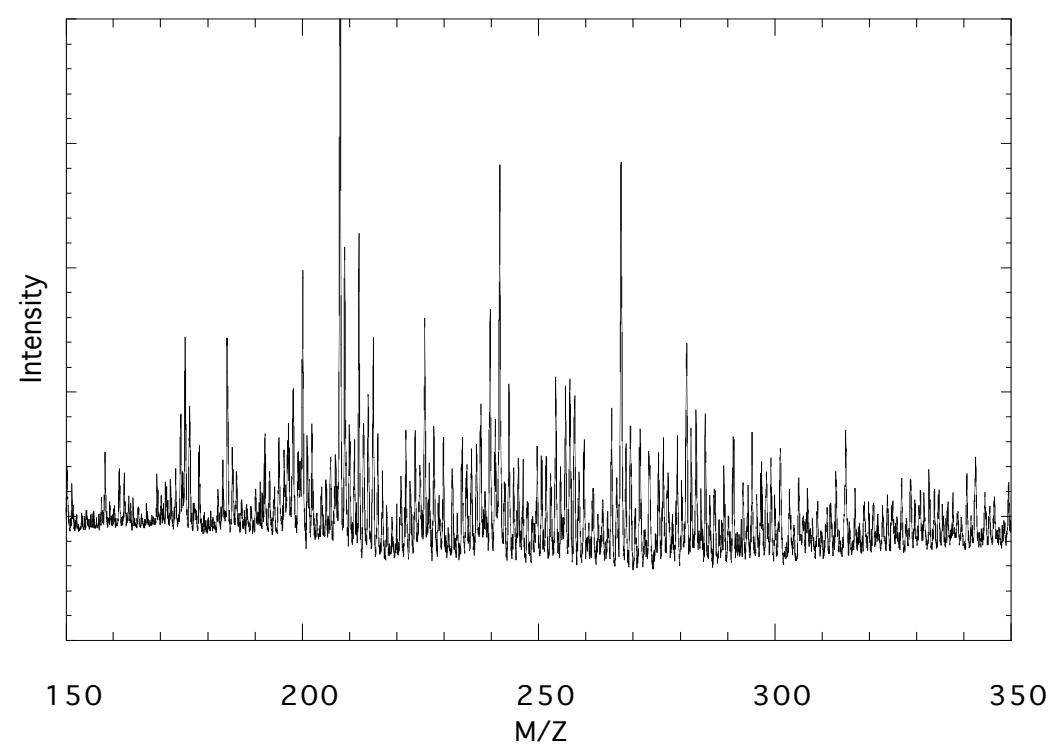

Figure 17. The laser desorption ( $\mathrm{L}^{2} \mathrm{MS}$ ) mass spectrum of an interstellar ice analog residue produced by the photolysis of a $\mathrm{H}_{2} \mathrm{O}: \mathrm{CH}_{3} \mathrm{OH}: \mathrm{NH}_{3}$ :CO (100:50:1:1) ice. The complexity of the interstellar ice analog residue is clearly evident. This work initially reported in [221]. 
the simple starting materials of $\mathrm{H}_{2} \mathrm{O}: \mathrm{CH}_{3} \mathrm{OH}: \mathrm{NH}_{3}: \mathrm{CO}$ has a mass greater than 32 nor do they contain a single $\mathrm{CC}$ bond, the complexity and extent of the photoproducts is staggering. Many of these compounds have molecular masses up to ten times larger than that of any of the starting materials.

When this mass spectrum is compared with those of two interplanetary dust particles (IDPs) there is an intriguing similarity between the mass envelope between roughly 150 to $300 \mathrm{M} / \mathrm{Z}$ and peaking near $220 \mathrm{M} / \mathrm{Z}$ characteristic of the interstellar ice residue shown in Figure 17 and the mass envelopes in the IDP spectra which span the range from about $200 \mathrm{M} / \mathrm{Z}$ to $300 \mathrm{M} / \mathrm{Z}$ and appear to peak near $250 \mathrm{M} / \mathrm{Z}$ (see e.g. [220]). As with the HPLC results above, this resemblance between the laboratory ice residue and the extraterrestrial organics brought to Earth - this time in IDPs - raises the question, "Do the compounds in IDPs have an interstellar ice/cometary heritage?"

\subsubsection{Complex Organic Production in Ices with PAHs}

As described in $\$ 2$, PAHs are one of the most abundant interstellar polyatomic molecules known in the gas and are widespread throughout the interstellar medium. As with all other polyatomic gas phase species in dense clouds, they should freeze out onto the grains and become part of the mixed molecular ice. During the past three years we also investigated the photochemical behavior of a few small PAHs in $\mathrm{H}_{2} \mathrm{O}$ ices to lay the foundation for a study of PAH photochemistry in realistic, multicomponent IS ice analogs. While the UV photolysis of PAHs in interstellar ices modifies only a small fraction of the interstellar PAH population, this change is significant in terms of interstellar chemistry and interesting from the prebiotic chemistry perspective. Upon photolysis in pure $\mathrm{H}_{2} \mathrm{O}$ ice at $10 \mathrm{~K}$, simple PAHs are not destroyed. Rather, they are partially oxidized and/or reduced (hydrogenated).[222] These PAH structures are illustrated in Figures 18 and 19. If deuterium is present in the ice, deuterated aromatics are also produced.[222,223] These alterations have significantly different effects on the chemical nature of the parent. Hydrogen atom addition transforms some of the edge rings into cyclic aliphatic hydrocarbons, thereby creating molecules with both aromatic and aliphatic character and decreasing the overall degree of aromaticity. Oxygenation produces ketones or aldehydes, changes which open up an entire range of possible
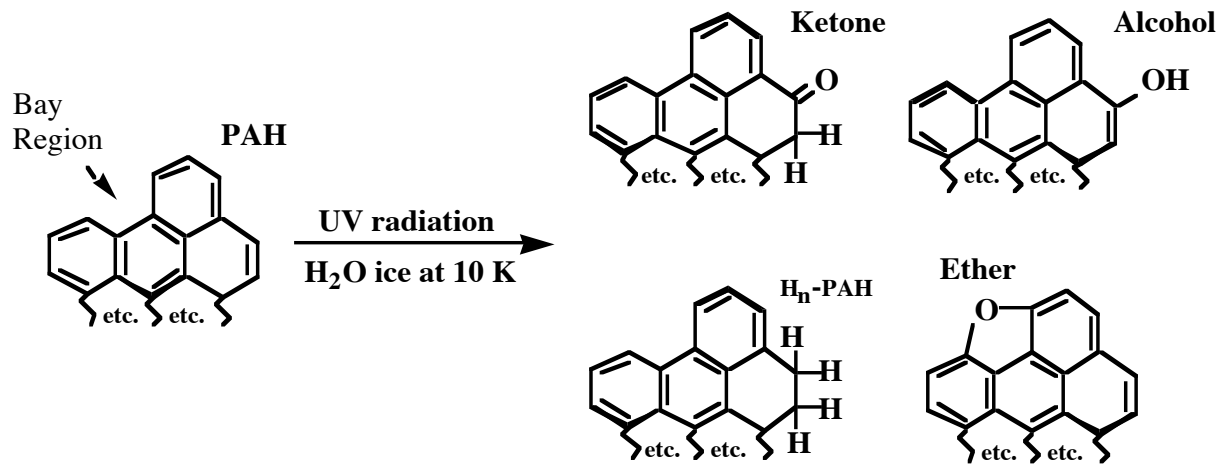

Figure 18. The types of PAH structures produced when PAHs are UV irradiated in water ice at 10 K.[222] 


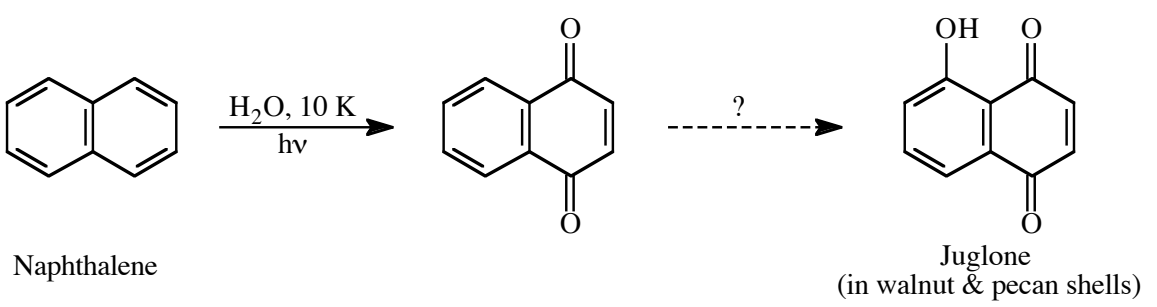

Figure 19. Molecules very similar to juglone, a compound synthesized and used by walnut trees can be produced abiotically in water-rich ices containing the aromatic molecule naphthalene.[224] The starting $\mathrm{PAH} / \mathrm{H}_{2} \mathrm{O}$ concentration of a few percent is conservatively lower than their observed relative abundance in the interstellar medium.[225-227]

chemical reactions that were not available to the parent PAH. Aromatic ketones (quinones) are of particular prebiotic interest since they are widely used in current living systems for electron transport across cell membranes. In view of PAH photochemistry in pure $\mathrm{H}_{2} \mathrm{O}$ ice, it seems plausible that aromatic structures decorated with alkyl, amino, hydroxyl, cyano, carboxyl, and other interesting functional groups may be produced when mixed-molecular ices containing PAHs are photolyzed.

\subsection{INTERSTELLAR PAHS, ICES AND THE ORIGIN OF LIFE}

Taking another approach to understanding the role these interstellar/precometary residues might have played in the chemistry on the early Earth and possibly the emergence of life, we are investigating their bulk or collective chemical properties in collaboration with Professor D. Deamer from the University of California, Santa Cruz. While much of the residue dissolves rapidly when added to liquid water or methanol, water-insoluble droplets are also formed.[206] Figure 20 shows a micrograph of these non-soluble droplets in water. Many of the droplets also show intriguing internal structures. Droplet formation shows that some of the complex organic compounds produced in these interstellar ice analogs are amphipillic, i.e. they have both a polar and non-polar component, similar in structure to the molecules which comprise soap. These are also the types of molecules that make up cell membranes and membrane production is considered a critical step in the origin of life. These droplets can encapsulate hydrophyllic fluorescent dyes within their interior, demonstrating that they are true vesicles (hollow droplets) with their interiors separated from surroundings by their lipid multilayer (see Fig 5 in [206]). Vesicle formation is thought critical to the origin of life since vesicles provide an environment in which life can evolve, isolating and protecting the process from the surrounding medium. For example, within the confines of a vesicle, $\mathrm{pH}$ can be moderated and held at a different value from that in the surrounding medium, and nutrients, catalysts, and other materials can be concentrated and held together. While it is uncertain where membrane formation falls in the sequence of events leading up to the origin of life, with some arguing that it must have been one of the first steps [228], and others that it occurred at a later stage [229], it is considered a very crucial step.

Figure 21 shows that the membranes trap other, photoluminescent, molecules that are also produced within the ice by UV irradiation.[206] Thus, not only are vesicle forming compounds produced from the simplest and most abundant IS starting 


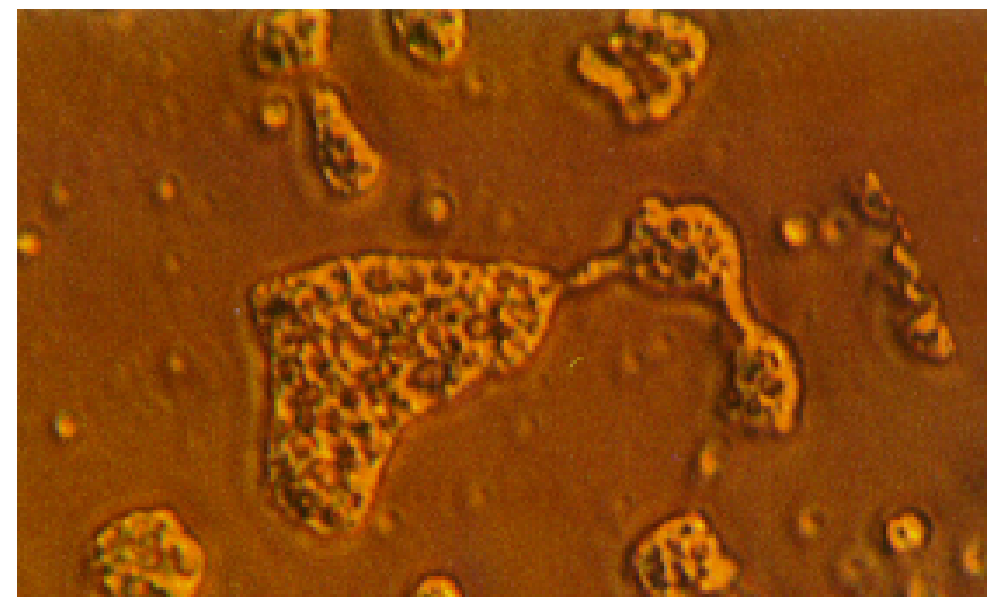

Figure 20. Phase micrograph of the water insoluble droplets formed when a few drops of water are placed on the organic residue produced from the UV photolysis of the realistic interstellar/precometary ice analog: $\mathrm{H}_{2} \mathrm{O}: \mathrm{CH}_{3} \mathrm{OH}: \mathrm{NH}_{3}: \mathrm{CO}(100: 50: 1: 1)$ at $10 \mathrm{~K}$. The addition of liquid water mimics what might happen within a comet or on a parent body when these materials are exposed to liquid water. The large structure is $10 \mu \mathrm{m}$ across. These selforganizing droplets also trap luminescent materials in their structures as shown in Figure 21B.[206]

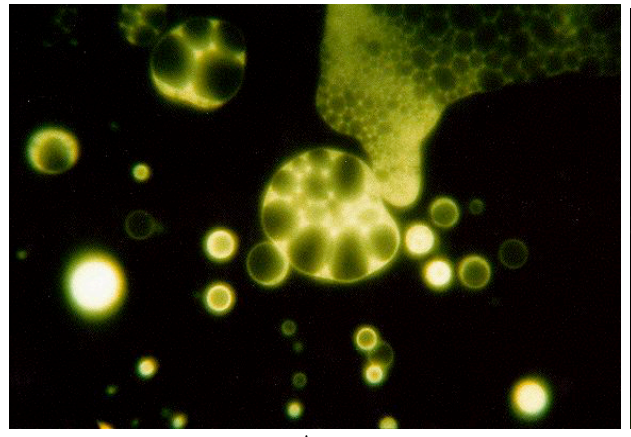

A

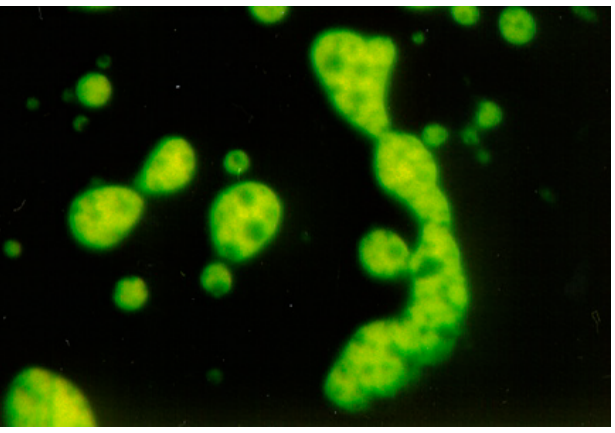

B

Figure 21. (A) Fluorescence micrograph of the water insoluble droplets formed from a Murchison meteorite extract [230] compared to (B) the fluorescent droplets produced from the photolysis residue of the interstellar/precometary ice analog: $\mathrm{H}_{2} \mathrm{O}: \mathrm{CHOH}: \mathrm{NH}: \mathrm{CO}(100: 50: 1: 1)$ at 10 K.[206]

materials, complex organics which absorb UV are also formed. This ability to form and trap energy receptors within these structures is considered another critical step in the origin of life as it provides the means to utilize energy available outside the system.

As shown in Figure 21, the residue droplet-forming behavior upon addition to liquid water is strikingly similar to that found for the organic components of the Murchison meteorite [230], another indication of similarity between the laboratory ice residue and extraterrestrial organics in meteorites.

Before ending with a discussion on the possible role that these materials which are produced by the energetic processing of IS ices might play in the origin of life, it is 
useful to first consider the evidence in favor of a strong connection between interstellar organic materials and the carbonaceous fractions of meteorites and IDPs (and by implication comets).[231,232] For example, complex organic molecules similar to those produced in our laboratory studies of photolyzed ices have been identified in meteorites $[233,234]$ and many oxidized polycyclic aromatic hydrocarbons are present in organic extracts from the Murchison meteorite.[235] Furthermore, the PAHs in meteorites are deuterium enriched [231] as is the carbon in IDPs.[236,237] The formation histories of these extraterrestrial materials are not well understood, although the presence of deuterium enrichments in many of the classes of these compounds has been taken to implicate an interstellar origin.[231,238] Likewise, the similarity between the interstellar ice constituents and relative abundances with known cometary constituents listed in Table 7 is remarkable (see e.g. [209,210]). Recent work continues to support this picture.

These similarities between interstellar ices and PAHs with the extraterrestrial materials delivered to the Earth strengthens the case for taking interstellar PAHs and ices into account when pondering an exogenous origin of life. Three roles that these IS materials might have played in the exogenous origin of life on Earth considered are: (1) A supplier of the basic, prebiotic raw materials from which biotic compounds were eventually produced on Earth; (2) The source of complex prebiotic materials poised to play a direct role once in a favorable environment; and (3) The fountainhead of some organic species and mixtures capable of carrying out the basic processes of life.

(1) Supplier of basic, raw materials. First, comets were very likely responsible for raining a rich inventory of potentially important prebiotic molecules down onto the early Earth. HMT and the family of related species provides an example. Recall the variety of species that are formed by HMT hydrolysis, thermalization, or photolysis, all processes which can occur during a comet's lifetime (\$3.2.1). For example, hydrolysis under acidic conditions yields ammonia and formaldehyde as well as amino acids. In addition to the possible prebiotic activity of HMT itself, its ice synthesis implicates the presence of several other biogenically interesting intermediates as well, including formaldehyde, ammonia, and cyano-containing species. The observed differential sublimation of these moderately volatile compounds in the laboratory may be similar to that which occurs when cometary ice fragments and dust grains are ejected into cometary comae.[145] This process of differential sublimation in the coma followed by photolysis can regulate the distribution of some of the molecular photofragments seen in cometary comae. Perhaps since ultraviolet photolysis of HMT frozen in $\mathrm{H}_{2} \mathrm{O}$ ice produces the $\mathrm{OCN}^{-}$band observed in the spectra of protostellar objects and laboratory ices, HMT may contribute to some of the $\mathrm{OCN}^{-}$in protostellar objects and, as such, contribute to the extended CN source in cometary comae.

(2) Source of complex prebiotic materials. Apart from supporting the hypothesis that meteorites, dust, and comets delivered organics to the early earth which served as a useful prebiotic chemical reservoir $[4,11,239]$, the laboratory work described here supports the idea that these extraterrestrial sources could have delivered species which were sufficiently complex to play a direct role in the chemistry of the origin of life and not only provided the raw materials. Section 3.2 described the intriguing behavior of some of the compounds produced by irradiation of realistic interstellar/precometary ice analogs. Some of these products have been found to self-organize to form membranes 
and ultimately vesicles when they are immersed in liquid water. Other species produced in the ice together with the vesicle forming compounds are trapped within these vesicles. These other organic molecules can harvest ultraviolet light, bringing energy from the surroundings into the vesicle and driving further chemistry. When PAHs, very abundant interstellar species, are included in the ice irradiation experiments, the level of chemical complexity in both structure and behavior rises significantly. Substituted aromatic structures are readily produced which are common in today's biological systems. Quinones, for example, are used to transport electrons across membrane walls in living systems. Also, many alkaloids have aromatic base skeletons. In addition, other interstellar ice simulation experiments are known to produce amino acids [214,240], as well as glycerol the precursors of simple sugars. Thus, comets might represent quite potent prebiotic cocktails. All of these complex materials are produced by the irradiation of simple ice mixtures that are known to be widespread throughout the galaxy in dense molecular clouds. Thus, it seems warranted to hypothesize that wherever a planet or satellite is formed in the habitable zone, it stands to accrete these chemical remnants from the nascent cloud from infalling IDPs, cometary (formerly interstellar) and meteoritic materials. If the emergence of life is indeed an increase in chemical complexity as is currently thought, one of the challenges before us is to understand this chemistry and to assess the relative importance of this input into the origin of life versus the build-up from the compounds already present on the primordial planet.

(3) Fountainhead of species undergoing the basic processes of life. Carrying this one step further, we end this article on a speculative note. These early results are quite startling when viewed from the perspective held not long ago that chemistry in space was very limited. In view of this and the fact that there are hundreds of residue compounds not yet analyzed, an intriguing possibility now supported by these results is the production, within the comet itself, of species poised to take part in the life process, or perhaps even at the earliest stages of what would be perceived of as a living system. This "jump-starting" of the life process by the introduction of such marginally biologically active species, into the "warm pond" on a habitable planet may not be as far fetched as it would at first seem. Although one could hardly imagine a better deep freeze than isolation within a comet, with temperatures measured in the ten's of degrees $\mathrm{K}$, there are repeated episodes of warming for periodic comets (such as Halley) when they approach the Sun. Since these warming episodes, which can be repeated many times in a given comet, can last anywhere from periods of weeks to several months, there is ample time for a very rich mixture of complex organics to develop even though these warming episodes are sandwiched between long periods of extreme cold. Since the surface becomes the warmest, above $300 \mathrm{~K}$ for comets which pass closer to the Sun than the Earth, most of the volatiles within a few meters of the surface sublime during each passage. During these warming periods, regions within the comet nucleus will be warmed to different temperatures as well. As the surface heat wave propagates inward, different processes can occur within different regions of the cometary nucleus depending on the maximum temperature and duration of the thermal pulse. In the deepest regions where the temperatures only rise to between 50 and $150 \mathrm{~K}$, diffusion limited reactions will be favored. At higher temperatures, these as well as other reactions will be allowed. It is even conceivable that liquid water might be present for short periods within the larger comets. These low temperatures and the conditions that ensure slow 
processing and gradual periods of warming and cooling may actually serve to protect any larger species formed much in the way membranes are involved for aqueous systems. At this early stage in our understanding of cometary ices it already appears that comets contain many of the types of compounds which are considered important players in the origin of life, compounds which play central physical roles and compounds which play key chemical roles. Indeed there is evidence for nearly every class of organic chemical thought important in the "RNA world" in these irradiated ices and they are all held in close proximity to one another. Consider also the unexplored avenues afforded by the additional internal energy available to drive chemistry as the ice undergoes transitions from one phase to the next, the crystal field effects, the structural and templateing restrictions the ice places on reactions and the forced stereochemistry. We are just beginning to appreciate the complexity and possibilities of these ices. Thus, it is no longer inconceivable that comets played a far more active role in the origin of life than simply that of a spectator-delivery system of raw materials. Perhaps Darwin's 'warm little pond' is a warmed comet.

\section{CONCLUSION}

The last two and a half decades of infrared spectroscopic observations of cosmic ices and PAHs, the two classes of materials we have learned most about through these observations, has taught us that these species are an integral part of the rich and complex world of Interstellar Chemistry. This chemistry includes contributions from gas phase ion-molecule reactions, radical driven reactions, surface reactions on grains, and solid state reactions initiated by energetic processing of icy grain mantles, to name but a few of the processes at work. Further, cometary observations and meteoritic and interplanetary dust particle (IDP) isotopic anomalies now indicate that some interstellar material could be delivered to planetary surfaces essentially unmodified. Taken together, these results are driving a very large shift in our understanding of the kinds of materials that might fall on primordial planetary systems. As recently as the 1960's, it was thought that interstellar conditions were too harsh for any significant polyatomic chemistry to take place. It was thought that any compounds that could form under the extremely low densities of space would quickly be dissociated by the plentiful highenergy radiation. Factors such as the dust extinction which moderates UV in dense clouds, the protection and chemical productivity afforded by ices, and the ubiquity of the UV-hardy aromatic hydrocarbons were simply not considered. Furthermore, it was thought that even if there was some chemical complexity prior to the Solar System's formation, the extreme temperatures and kinetic violence of that process would have erased any significant chemical memory of interstellar input. Indeed, the aromatic hydrocarbons in meteorites were interpreted to be the end products of simpler hydrocarbons heated above 300 to $400 \mathrm{C}$ on the various parent bodies during star and planet formation. Today we are beginning to see that PAHs and related materials are abundant throughout the ISM; that the simplest interstellar ices can foster the production of a stunning array of complex organic species; and that copious quantities of these materials can not only survive the rigors of the star formation process, but also deposition onto the resultant planetary bodies. Thus, we are in the midst of a major shift in our understanding of what contributed to the 'atmospheres' of the terrestrial planets. Instead of simply assuming that a planet's prebiotic chemistry had to 'start 
from scratch', evidence is building that a far more chemically complex interstellar broth was present right from the start.

This more recent perspective requires much work before its role can be fully assessed. For example, "How important is it?" is a key question. In view of the size of the terrestrial planets and the amount of materials initially present, how significant a role could this extraterrestrial input have played anyway? Conversely, the size of the terrestrial planets might be viewed as a disadvantage to generating chemical complexity just as the low densities of space do not favor the production of complex polyatomic molecules. In both cases, the low concentrations of organic materials hampers such reactions. From this perspective, the chemical intimacy of an interstellar and cometary ice might have great advantage.

Interstellar ice composition is most relevant for those comets which preserve their IS ice heritage and IDPs and meteorites which preserve interstellar organic refractory materials. IS ice composition depends on local conditions. In areas associated with star, planet, and comet formation, ices comprised of simple, polar species and entrapped volatiles such as $\mathrm{H}_{2} \mathrm{O}, \mathrm{CH}_{3} \mathrm{OH}, \mathrm{CO}, \mathrm{CO}_{2}, \mathrm{NH}_{3}$, and $\mathrm{H}_{2} \mathrm{CO}$ are most important. Photolysis of these ices produces the simple species $\mathrm{H}_{2}, \mathrm{H}_{2} \mathrm{CO}, \mathrm{CO}_{2}, \mathrm{CO}, \mathrm{CH}_{4}, \mathrm{HCO}$ as well as an extremely rich mixture of complex organic molecules. We now know that this prebiotic cocktail includes unsaturated fluorescent compounds; self-assembling, amphiphillic molecules; sugar precursors; and amino acids and we have just scratched the surface. These same lines of sight also show evidence for polycyclic aromatic hydrocarbons (PAHs), the organic molecules known from their characteristic emission to be widespread and abundant throughout the interstellar medium. Including PAHs in the irradiated ices produces aromatic alcohols, quinones and ethers, species similar to those found in meteorites and having similar chemical properties to functionalized aromatic species used in living systems today. All of the above compounds are readily formed and thus likely cometary constituents at the 0.1 to 1 percent level. Evidence is growing that this interstellar heritage did not become erased during the Solar Nebula phase, implying that this chemical inventory contributed to the chemistry on the primitive Earth. The ready formation of these organic species from simple starting mixtures under general interstellar conditions, the ice chemistry that ensues when these ices are mildly warmed, and the observation that the more complex refractory photoproducts form fluorescent vesicles upon exposure to liquid water underscore the possibility that IS and cometary ices could have played an important role in the origin of life. Three possibilities are considered, ranging from these interstellar materials simply providing the raw materials used for a completely endoginous origin to the opposite extreme that they delivered species poised to take part in the life process, or perhaps even at the earliest stages of what would be perceived of as a living system. While far-fetched to many of us only a few years ago, the evidence suggests that this is conceivable.

\section{References}

[1] Allamandola, L.J. and Tielens, A.G.G.M. (eds.), (1989) Interstellar Dust, Proceedings of IAU Symposium 135, Kluwer Academic Pub., Dordrecht.

[2] Pirronello, V., and Krelowski, J. (eds.), (2001) Solid State Astrochemistry, Kluwer Academic Pub., Dordrecht. 
[3] Herbst, E. (2001), in Pirronello, V., and Krelowski, J. (eds.), Solid State Astrochemistry, Kluwer Academic Pub., Dordrecht (this volume).

[4] Greenberg J.M., (2001), in Pirronello, V., and Krelowski, J. (eds.), Solid State Astrochemistry, Kluwer Academic Pub., Dordrecht (this volume).

[5] Williams D.A., (2001), in Pirronello, V., and Krelowski, J. (eds.), Solid State Astrochemistry, Kluwer Academic Pub., Dordrecht (this volume).

[6] Winnewisser, G. (1997), in Cosmovici, C.B., Bowyer, S., and Werthimer, D. (eds.), Astronomical and Biochemical Origins and the Search for Life in the Universe, Editrice Compositori of the Italian Physical Society, Bologna, p.5.

[7] Irvine, W.M. (1998) Orig. Life Evol. Biosphere, 28, 365.

[8] Sandford, S.A. and Allamandola L.J. (1993) Ap.J., 417, 815 and references therein.

[9] Charnley, S.B., Tielens, A.G.G.M., and Millar T.J. (1992) Ap.J., 399, L71.

[10] Charnley, S.B. (1997), in Cosmovici, C.B., Bowyer, S., and Werthimer, D. (eds.), Astronomical and Biochemical Origins and the Search for Life in the Universe, Editrice Compositori of the Italian Physical Society, Bologna, p.89.

[11] Oro, J., and Cosmovici, C.B. (1997), in Cosmovici, C.B., Bowyer, S., and Werthimer, D. (eds.), Astronomical and Biochemical Origins and the Search for Life in the Universe, Editrice Compositori of the Italian Physical Society, Bologna, p.97.

[12] Gillett, F.C., Forrest, W.J., and Merrill, K.M. (1973) Ap J., 183, 87.

[13] Russell, R.W., Soifer, B.T., and Willner, S.P. (1977) Ap J., 217, L149 and references therein.

[14] Aitken, D.K. (1981), in Wynn-Williams C.G., and Cruikshank, D.P. (eds.), Infrared Astronomy, D. Reidel Pub. Co., Dordrecht, p. 207 and references therein.

[15] Willner, S.P. (1984), in Kessler, M.F., and Phillips, J.P., (eds.), Galactic and Extragalactic Infrared Spectroscopy, D. Reidel Publishing Co., Dordrecht, , p. 37 and references therein.

[16] Phillips, M.M., Aitken, D.K., and Roche, P.F. (1984) MNRAS, 207, 25 and references therein.

[17] Special Edition: First Results from ISO (1996) $A \& A, \mathbf{3 1 5}(2)$.

[18] Puget, J.L. (1989) in Allamandola, L.J., and Tielens, A.G.G.M. (eds.), Interstellar Dust, Kluwer Academic Pub., Dordrecht, p. 119.

[19] Leger, A., \& Puget, J.L. (1984) A\&A, 137, L5.

[20] Allamandola, L.J., Tielens, A.G.G.M., and Barker, J.R. (1985) Ap.J., 290, L25.

[21] Genzel, R., et al. (1998) Ap.J., 498, 579.

[22] Roelfsma, P.R., et al. (1996) A\&A, 315, L289.

[23] Greenberg, J.M. (1971) A\&A, 12, 240.

[24] Purcell, E.M., (1976) Ap.J., 206, 685.

[25] Sellgren, K. (1984) Ap.J., 235, 138.

[26] M. Cohen et al. (1986) Ap.J., 302, 737.

[27] M. Cohen et al. (1989) Ap.J., 341, 246.

[28] Tielens, A.G.G.M. et al. (1993) Science, 262, 86.

[29] d'Hendecourt, L.B., and Leger, A. (1987) A\&A, 180, L9.

[30] Lepp, S., and Dalgarno, A. (1988) Ap.J., 324, 553.

[31] Omont, A. (1986) $A \& A, \mathbf{1 6 4}, 159$. 
[32] Allamandola, L.J., Sandford, S.A., and Wopenka, B. (1987) Science, 237, 56.

[33] Allamandola, L.J., Tielens, A.G.G.M., and Barker, J.R. (1989) Ap.J.Supp.Ser., 71, 733.

[34] DeVries, M.S., et al. (1993) Geochim.Cosmochim. Acta 57, 933.

[35] Clemmett, S., Maechling, C., Zare, R., Swan, P., and Walker, R. (1993) Science, 262, 721.

[36] van der Zwet, G.P., and Allamandola, L.J. (1985) A\&A, 146, 76.

[37] Leger, A., and d'Hendecourt, L.B. (1985) A\&A, 146, 81.

[38] Crawford, M.K., Tielens, A.G.G.M., and Allamandola, L.J. (1985) Ap.J., 293, L45.

[39] Allamandola, L.J., Tielens, A.G.G.M., and Barker, J.R. (1989) Ap.J.Supp.Ser., 71, 733.

[40] Puget, J.L., and Leger, A. (1989) Ann.Rev.Astron.Astrophys., 27, 161.

[41] Allamandola, L.J. (1996), in Greenberg, J.M. (ed.), The Cosmic Dust Connection, Kluwer Academic Pub., Dordrecht, p.81.

[42] Sloan, G., Grasdalen, G., and LeVan, P. (1993) Ap.J., 409, 412.

[43] Bregman, J., Rank, D., Temi, P., Hudgins, D., and Kay, L. (1993) Ap.J., 411, 794.

[44] Bregman, J., Rank, D., Sandford, S., and Temi, P. (1993) Ap.J., 410, 668.

[45] Bernstein, M.P., Sandford, S.A., and Allamandola, L.J. (1996) Ap.J., 472, L127,

[46] Sloan, G.C., Bregman, J.D., Geballe, T.R., Allamandola, L.J. and Woodward, C.E. (1996) Ap.J., 474, 735.

[47] Sloan, G.C., Hayward, T.L., Allamandola, L.J., Bregman, J.D., DeVito, B., and Hudgins, D.M. (1999) Ap.J., 513, L65.

[48] Flickinger, G.C., and Wdowiak, T.J. (1990) Ap.J., 362, L71.

[49] Flickinger, G.C., Wdowiak, T.J., and Gomez, P.L. (1991) Ap.J., 380, L43.

[50] Brenner, J.D. and Barker, J.R. (1992) Ap.J., 388, L39.

[51] Kurtz, J. (1992), $A \& A, \mathbf{2 5 5}, \mathrm{L} 1$.

[52] Joblin, C., Boissel, P., Leger, A., d'Hendecourt, L.B., and Defourneau, D. (1995) $A \& A, 299,835$.

[53] Barker, J.R., and Cherchneff, I. (1989), in Allamandola, L.J., and Tielens, A.G.G.M., (eds.), Interstellar Dust: Proceedings of IAU Symposium 135, Kluwer Academic Pub., Dordrecht, p.197.

[54] Leger, A., d'Hendecourt, L.B., Defourneau, D. (1989), A\&A 216, 148.

[55] Salama, F., Bakes, E., Allamandola, L.J., and Tielens, A.G.G.M. (1996) Ap.J., 458, 621 .

[56] Salama, F., Galazutdinov, G.A., Krelowski, J., Allamandola, L.J., and Musaev, F.A. (1999) Ap.J., 526, 265.

[57] Hudgins, D., Sandford, S.A., and Allamandola, L.J. (1994) J.Phys.Chem., 98, 4243.

[58] Hudgins, D.M., and Allamandola, L.J. (1995) J.Phys.Chem., 99, 3033.

[59] Halasinski, T., Hudgins, D.M., Salama, F., Allamandola, L.J., and Bally, T. (2000) J.Phys.Chem., 104, 7484.

[60] Schutte, W., Tielens, A.G.G.M., and Allamandola, L.J. (1993) Ap.J., 415, 397.

[61] Cook, D.J., and Saykally, R.J. (1998) Ap.J., 493, 793.

[62] Hudgins, D.M., and Allamandola, L.J. (1995) J.Phys.Chem., 99, 8978. 
[63] Hudgins, D.M., and Allamandola, L.J. (1997) J.Phys.Chem., 101, 3472.

[64] Hudgins, D.M., and Sandford, S.A. (1998) J.Phys.Chem.A, 102, 329.

[65] Hudgins, D.M., and Sandford, S.A. (1998) J.Phys.Chem.A, 102, 344.

[66] Hudgins, D.M., and Sandford, S.A. (1998) J.Phys.Chem.A, 102, 353.

[67] Hudgins, D.M., Bauschlicher, C.W., Jr., Allamandola, L.J., and Fetzer, J.C. (2000) J.Phys.Chem.A, 104, 3655.

[68] Bauschlicher, C.W., Jr., Langhoff, S.R., Sandford, S.A., and Hudgins, D.M. (1997) J.Phys.Chem.A, 101, 2414.

[69] Langhoff, S.R. Bauschlicher, C.W., Jr., Hudgins, D.M., Sandford, S.A., and Allamandola, L.J. (1998) J.Phys.Chem.A, 102, 1632.

[70] Hudgins, D.M., Bauschlicher, C.W., and Allamandola, L.J. (2001) Spectrochim.Acta.A, 57, 907.

[71] Mattioda, A., Hudgins, D.M., Bauschlicher, C.W., Jr., and Allamandola, L.J. (manuscript in preparation).

[72] Szczepanski, J., Roser, D., Personette, W., Eyring, M., Pellow, R., and Vala, M. (1992) J.Phys.Chem., 96, 7876.

[73] Szczepanski, J., and M. Vala, Nature 363, 699 (1993)

[74] Szczepanski, J., and Vala, M. (1993) Ap.J., 414, 179.

[75] Szczepanski, J., Chapo, C., and Vala, M. (1993) Chem Phys, 205, 434.

[76] Szczepanski, J., Vala, M., Talbi, D., Parisel, O., Ellinger, Y.J. (1993) J.Chem.Phys., 98, 4494.

[77] Vala, M., Szczepanski, J., Pauzat, F., Parisel, O., Talbi, D, and Ellinger, Y.J. (1994) J.PhysChem., 98, 9187.

[78] Szczepanski, J., Wehlburg, C., and Vala, M. (1995) Chem.Phys.Lett., 232, 221.

[79] Szczepanski, J., Drawdy, J., Wehlburg, C., Vala, M. (1995) Chem.Phys.Lett., 245, 539.

[80] Talbi, D., Pauzat, F., Ellinger, Y. (1993) A\&A, 268, 805.

[81] de Frees, D.J., Miller, M.D., Talbi, D., Pauzat, F., and Ellinger, Y. (1993) Ap.J., 408, 530.

[82] Pauzat, F., Talbi, D., and Ellinger, Y. (1995) A\&A, 293, 263.

[83] Pauzat, F., Talbi, D., and Ellinger, Y. (1997) $A \& A, \mathbf{3 1 9}, 318$.

[84] Pauzat, F., Talbi, D., and Ellinger, Y. (1999) MNRAS, 304, 241.

[85] Pauzat, F., and Ellinger, Y. (2001) MNRAS, 324, 355.

[86] Langhoff, S.R. (1996) J.Phys.Chem., 100, 2819.

[87] Bauschlicher, C.W., and Langhoff, S.R. (1997) Spectrochim.Acta A, 53, 1225.

[88] Bauschlicher, C.W. (1998) Chem.Phys., 233, 29.

[89] Bauschlicher, C.W., and Langhoff, S.R. (1998) Chem.Phys., 234, 79.

[90] Bauschlicher, C.W., (1998) Chem.Phys., 234, 87.

[90] Bauschlicher, C.W. (1998) Chem.Phys., 234, 87.

[91] Bauschlicher, C.W., and and Bakes, E.L.O. (2000) Chem.Phys., 262, 285.

[92] Piest, J.A., von Helden, G., and Meijer, G. (1999) Ap.J. 520, L75.

[93] Piest, J.A., Oomens, J., Bakker, J., von Helden, G., and Meijer, G. (2001) Spectrochim.Acta A, 57A, 717.

[94] Schlemmer, S., Cook, D.J., Harrison, J.A., Wurfel, B., Chapman, W., and Saykally, R.J. (1994) Science, 265, 1686.

[95] (a) Wagner, D.R., Kim, H.-S., and Saykally, R.J. (2000) Ap.J., 545, 854; (b) Kim, H.-S., Wagner, D.R., and Saykally, R.J. Ap.J., (in press, 2001). 
[96] Schutte, W., Tielens, A.G.G.M., and Allamandola, L.J. (1993) Ap.J., 415, 397.

[97] Allamandola, L.J., Hudgins, D.M., and Sandford, S.A. (1999) Ap.J., 511, L115.

[98] Bregman, J.D., Allamandola, L.J., Tielens, A.G.G.M., Geballe, T.R., and Witteborn, F.C. (1989) Ap.J., 344, 791.

[99] Gauger, A., Gail, H.P., and Sedlmayr, E. (1990) $A \& A, \mathbf{2 3 5}, 345$.

[100] Cherchneff, I., Barker, J.R., and Tielens, A.G.G.M. (1992) Ap.J., 401, 269.

[101] Cadwell, B.J., Wang, H., Feigelson, E.D., and Frenklach, M. (1994) Ap.J., 429, 285.

[102] Buss, Jr., R.H., et al. (1993) Ap.J., 415, 250.

[103] Kwok, S., Hrivnak, B.J., and Geballe, T.R. (1995) Ap.J., 454, 394.

[104] Bregman, J.D. (1989), in Allamandola, L.J., and Tielens, A.G.G.M. (eds.) Interstellar Dust, Kluwer Acabemic Pub., Dordrecht, p.109.

[105] Blanco, A., Bussoletti, E., and Colangeli, L. (1988) Ap.J., 334, 875.

[106] Allamandola, L.J., Tielens, A.G.G.M., and Barker, J.R. (1987), in Leger, A., d'Hendecourt, L.B., and Boccara, N. (eds.) Polycyclic Aromatic Hydrocarbons and Astrophysics, D. Reidel Pub. Co., Dordrecht, p.255.

[107] Van Kerckhoven, C., et al. (2000) A\&A, 357, 1013.

[108] Moutou, C., Verstraete, L., Leger, A., Sellgren, K., and Schmidt, W. (2000) $A \& A, 354, \mathrm{~L} 17$.

[109] Hony, S., Van Kerckhoven, C., Peeters, E., Tielens, A.G.G.M., Hudgins, D.M. and Allamandola, L.J. (2001) A\&A., 370, 1030.

[110] Bauschlicher, C.W., Jr., private communication.

[111] Bellamy, L.J. (1958) The Infrared Spectra of Complex Organic Molecules, John Wiley \& Sons, New York.

[112] Hudgins, D.M., and Allamandola, L.J. (1999) Ap.J., 516, L41.

[113] Cohen, M., Tielens, A.G.G.M., and Allamandola, L.J. (1985) Ap.J., 299, L93.

[114] Roche, P.F., Aitken, D.K., and Smith, C.H. (1989) MNRAS 236, 485.

[115] Witteborn, F.C., Sandford, S.A., Bregman, J.D., Allamandola, L.J., Cohen, M., Wooden, D., and Graps, A.L. (1989) Ap.J., 341, 270.

[116] Allamandola L.J., Sandford S.A., Tielens A.G.G.M., and Herbst T.M. (1993) Science, 260, 64.

[117] Chiar J.E., Gerakines P.A., Whittet D.C.B., Pendleton Y.J., Tielens A.G.G.M., and Boogert, A.C.A. (1998) Ap.J., 498, 716.

[118] Ehrenfreund, P. (2001), in Pirronello, V., and Krelowski, J. (eds.), Solid State Astrochemistry, Kluwer Academic Pub., Dordrecht (this volume).

[119] Sandford, S.A. (1996) Met.\&Planet.Sci., 31, 449.

[120] Dorschner, J.. and Henning. Th.. (1995) Astron.Astrophys.Rev. 6, 271.

[121] van Dishoeck, E.F., Blake, G.A., Draine, B.T. and Lunine, J.I. (1993), in Levy, E.H., and Lunine, J.I. (eds.), Protostars and Planets III, Univ. of Arizona Press, Tucson, Arizona, p. 163.

[122] Sandford, S.A., and Allamandola, L.J. (1993) Ap.J. 417, 815.

[123] Greenberg, J.M. (1978), in McDonnell, J.A.M. (ed.), Cosmic Dust, John Wiley and Sons, New York, p.187.

[124] Tielens, A.G.G.M., and Hagen, W. (1982) A\&A. 114, 245.

[125] d'Hendecourt, L.B., Allamandola, L.J., and Greenberg, J.M. (1985) A\&A 152, 130. 
[126] Greenberg, J.M. (1989), in Allamandola, L.J., and Tielens, A.G.G.M. (eds.), Interstellar Dust, Kluwer Academic Pub., Dordrecht, p. 345.

[127] Charnley, S.B., Rodgers, S.D., and Ehrenfreund, P. (2001) A\&A. (in press).

[128] Moore, M.H., Donn, B., Khanna, R., and A'Hearn, M.F. (1983), Icarus, 54, 388.

[129] Moore, M.H., Ferrante, R.F., and Nuth, J.A. (1996) Planet.Spa.Sci., 44, 927.

[130] Bernstein, M.P., Sandford S.A., Allamandola L.J., Chang S. and Scharberg M.A. (1995) Ap.J., 454, 327.

[131] Pirronello, V. and Averna, D. (1988) A\&A, 196, 201.

[132] Pirronello, V. (1997), in Cosmovici, C.B., Bowyer, S., and Werthimer, D. (eds.), Astronomical and Biochemical Origins and the Search for Life in the Universe, Editrice Compositori of the Italian Physical Society, Bologna, p. 77.

[133] Pirronello, V., Liu, C., Shen, L., and Vidalli, G. (1997) Ap.J., 475, L69.

[134] Gerakines, P.A., Schutte, W.A., and Ehrenfreundt, P., (1995) A\&A, 312, 289.

[135] Palumbo, M.E., Pendleton, Y.J., and Strazzulla, G. (2000) Ap.J., 542, 890.

[136] Hiraoka, K., Miyagoshi, T., Takayama, T., Yamamoto, K., and Kihara, Y. (1998) Ap.J., 498, 710.

[137] Schutte, W. A. (1999), in Ehrenfreund, P. et al. (ed.) Laboratory Astrophysics and Space Research, Kluwer Academic Pub., Dordrecht, p.69.

[138] Charnley, S. (2001) Ap.J., (in-press).

[138] Chiar, J.E., Adamson, A.J., Kerr, T.H., and Whittet, D.C.B. (1994) Ap.J., 426, 240.

[139] Ohishi, M., Irvine, W.M., and Kaifu, N. (1992), in Singh, P.D. (ed.) Astrochemistry of Cosmic Phenomena, Kluwer Academic Pub., Dordrecht, p.171.

[140] Blake, G.A., Sutton, E.C., Masson, C.R., and Phillips, T.G., (1987) Ap.J., 315, 621 .

[141] van Dishoek, E., et al. (1996) A\&A, 315, L349.

[142] Allamandola, L.J., Sandford, S.A., Tielens, A.G.G.M., and Herbst, T.M. (1992) Ap.J., 399, 134.

[143] DeGrauw, T., et al. (1996) $A \& A, \mathbf{3 1 5}, \mathrm{L} 345$.

[144] Boogert, A.C.A., et al. (2000) A\&A, 353, 349.

[145] Allamandola, L.J., Sandford, S.A., and Valero, G. (1988) Icarus, 76, 225.

[146] Allamandola, L.J., and Sandford, S.A. (1988), in Bailey, M.E., and Williams, D.A. (eds.), Dust in the Universe, Cambridge Univ. Press, Cambridge, p.229.

[147] Willner, S.P. (1977) Ap.J., 214, 706.

[148] Capps, R.W., Gillett, F.C., and Knacke, R.F. (1978) Ap.J., 226, 863.

[149] Tielens, A.G.G.M., Allamandola, L.J., Bregman, J., Goebel, J., d'Hendecourt, L.B., and Witteborn, F.C. (1984) Ap.J., 287, 697.

[150] Bowey, J.E., Adamson, A.J., and Whittet, D.C.B. (1998) MNRAS, 298, 131.

[151] Lacy, J.H., et al. (1984) Ap.J. 276, 533.

[152] Ehrenfreund, P., Dartois, E., Demyk, K., and d'Hendecourt, L.B. (1998) A\&A, 239, L17.

[153] Schutte, W. A., et al. (1999) $A \& A, \mathbf{3 4 3}, 966$.

[154] Teixeira, T.C., Devlin, J.P., Buch, V., and Emerson, J.P., (1999) $A \& A, 347$, L19.

[155] Allamandola, L.J., Bernstein, M.P., and Sandford, S.A. (1997), in Cosmovici, C.B., Bowyer, S., and Werthimer, D. (eds.), Astronomical and Biochemical 
Origins and the Search for Life in the Universe, Editrice Compositori of the Italian Physical Society, Bologna, p 23.

[156] Altwegg, K., Balsiger, H., and Geiss, J. (1999), in Altwegg, K, Ehrenfreund, P., Geiss, J., and Huebner, W. (eds.) The Composition and Origin of Cometary Materials, Kluwer Academic Pub., Dordrecht, p.3.

[157] Crovisier, J., and Bocklee-Morvan, D. (1999), in Altwegg, K, Ehrenfreund, P., Geiss, J. and Huebner, W. (eds.) The Composition and Origin of Cometary Materials, Kluwer Academic Pub., Dordrecht, p.19.

[158] Gillett, F.C., and Forrest, W.J. (1973) Ap.J., 179, 483.

[159] Gillett, F.C., Jones, T.W., Merrill, K.M., and Stein, W.A. (1975) $A \& A, \mathbf{4 5}$, 77.

[160] Cox, P. (1989) A\&A, 225, L1.

[161] Omont, A., et al. (1990). Ap.J., 355, L27.

[162] Hagen, W., Tielens, A.G.G.M., and Greenberg, J.M. (1983) A\&A Supp.Ser., 51, 389.

[163] Knacke, R.F., McCorkle, S., Puetter, R.C., Erickson, E.F. and Kratschmer, W. (1982) Ap.J., 260, 141.

[164] Leger, A., Gauthier, S., Defourneau, D., and Rouan, D. (1983) A\&A, 117, 164.

[165] Smith, R.G., Sellgren, K., and Tokunaga , A.T. (1989) Ap.J., 344, 413.

[166] Smith, R.G., Sellgren, K., and Brooke, T.Y. (1993) MNRAS, 263, 749.

[167] Sato, S., Nagata, T., Tanaka, M. and Yamamoto, T. (1990) Ap.J., 359, 192.

[168] Hagen, W., Allamandola, L.J., and Greenberg, J.M. (1980) A\&A, 86, L3.

[169] Tielens, A.G.G.M., and Allamandola, L.J. (1987), in Morfill, G.E., and Scholer, M. (eds. ), Physical Processes in Interstellar Clouds, D. Reidel Pub. Co., Dordrecht, p. 333.

[170] Grim, R.J.A., Baas, F., Geballe, T.R., Greenberg, J.M., and Schutte, W.A. (1991) $A \& A, \mathbf{2 4 3}, 473$.

[171] Skinner, C.L., Tielens, A.G.G.M., Barlow, M.J., and Justtanont, K. (1992) Ap.J., 399, L79.

[172] Sandford, S.A., Allamandola, L.J., and Geballe, T.R. (1993) Science, 262, 400.

[173] Dartois, E., Schutte, W., Geballe, T.R., Demyk, K., Ehrenfreund, P., and d'Hendecourt, L.B. (1999) $A \& A, \mathbf{3 4 2}$, L32.

[174] Sandford, S.A., Allamandola, L.J., Tielens, A.G.G.M., and Valero, G. (1988) Ap.J., 329, 498.

[175] Elsila, J., Allamandola, L.J., and Sandford, S.A. (1997) Ap.J., 479, 818.

[176] Palumbo, M.E. (1997) J.Phys.Chem.A, 101, 4298.

[177] Palumbo, M.E., and Strazzulla, G. (1993), A\&A, 269, 568.

[177] Palumbo, M.E., and Strazzulla, G. (2000) Ap.J., 542, 894.

[178] Ehrenfreund, P., Boogert, A.C.A., Gerakines, P.A., Jansen, D.J., Schutte, W.A., Tielens, A.G.G.M., and van Dishoek, E.F. (1996) A\&A, 315, L341

[179] Geballe, T.R. (1986) A\&A., 162, 248.

[180] Eiroa, C., and Hodapp, K.-W. (1989) A\&A., 210, 345.

[181] Tielens, A.G.G.M., Tokunaga, A.T., Geballe, T.R., and Baas, F. (1991) Ap.J., 381, 181.

[182] Whittet, D.C.B., Adamson, A.J., Duley, W.W., Geballe, T.R., and McFadzean, A.D. (1989) MNRAS, 241, 707.

[184] Chiar, J.E., Adamson, A.J., Kerr, T.H., and Whittet, D.C.B. (1995) Ap.J., 455, 234. 
[185] Chiar, J.E., Pendleton, Y.J., Geballe, T.R., and Tielens, A.G.G.M. (1998) Ap.J., 507, 281.

[186] Tegler, S.C., Weintraub, D.A., Allamandola, L.J., Sandford, S.A., Rettig, T.W., and Campins, H. (1993) Ap.J., 411, 260.

[187] Weintraub, D.A., Tegler, S.C., Kastner, J.H., and Rettig, T. (1994) Ap.J., 423, 674.

[188] Tegler, S.C., Weintraub, D.A., Rettig, T.W., Pendleton, Y.J., Whittet, D.C.B., and Kulesa, C.A. (1995) Ap.J., 439, 279.

[189] Pendleton, Y.J., Tielens, A.G.G.M., Tokunaga, A.T., and Bernstein, M.P. (1999) Ap.J., 513, 294.

[190] Whittet, D.C.B., Pendleton, Y.J., Gibb, E.L., Boogert, A.C.A., Chiar, J.E. and Nummelin, A. (2001) Ap.J., 550, 793.

[191] Grim, R.J.A., and Greenberg, J.M. (1987) Ap.J., 321, L91.

[192] Schutte, W.A., and Greenberg, J.M. (1997) A\&A., 317, L43.

[193] Demyk, K., Dartois, E., d'Hendecourt, L.B., Jourdain de Muizon, M., Heras, A., Breitfellner, M. (1998) A\&A, 339, 553.

[194] Palumbo, M.E., Pendleton, Y.J. and Strazzulla, G. (2001) Ap.J., 542, 890

[194] Hudson, R.L., Moore, M.H., and Gerakines, P.A. (2001) Ap.J. (in-press)

[195] Bernstein, M.P., Sandford, S.A., and Allamandola, L.J. (2001) Ap.J., 542, 894.

[195] d'Hendecourt, L.B., and Jordain de Muizon, M. (1989) A\&A, 223, L5.

[196] Ehrenfreund, P., Boogert, A.C.A., Gerakines, P.A., Tielens, A.G.G.M., and van Dishoek, E.F. (1997) $A \& A, \mathbf{3 2 8}, 649$.

[197] Gerakines, P.A., et al. (1999) Ap.J., 522, 357.

[198] Sandford, S.A., and Allamandola, L.J. (1990) Ap.J., 355, 357.

[199] Sandford, S.A., Bernstein, M.P., Allamandola, L.J., Goorvitch, D., and Teixeira, T.C.V.S. (2001) Ap.J., 548, 836.

[200] Dartois, E., Demyk, K., d'Hendecourt, L.B., and Ehrenfreund, P. (1999) A\&A., 351, 1066.

[201] Lacy, J., Carr, J., Evans, N., Baas, F., Achtermann, J., and Arens, J. (1991) Ap.J., 376, 556.

[202] Boogert, A.C.A., Schutte, W.A., Helmich, F.P., Tielens, A.G.G.M., and Wooden, D.H. (1997) A\&A., 317, 929.

[203] Lacy, J., Faraji, H., Sandford, S.A., and Allamandola, L.J. (1998): Ap.J., 501, L105.

[204] Schutte, W.A., Gerakines, P.A., Geballe, T.R., van Dishoek, E.F., and Greenberg, J.M. (1996) A\&A, 309, 633.

[205] Palumbo, M.E., Tielens, A.G.G.M., and Tokunaga, A.T. (1995) Ap.J., 449, 674.

[206] Dworkin, J.P., Deamer, D.W., Sandford, S.A., and Allamandola, L.J. (2001) Pub.Nat.Acad, 98, 815.

[207] Charnley, S.B., Tielens, A.G.G.M., and Millar, T.J. (1992) Ap.J., 399, L71.

[208] Blake, D., Allamandola, L.J., Sandford, S.A., Hudgins, D.M., and Freund, F. (1991) Science, 254, 548.

[209] Mumma, M.J., Stern, S.A., and Weissman, P.R. (1993), in Levy, E.H., Lunine, J.I., and Matthews, M.S. (eds.), Protostars and Planets III, University of Arizona Press, Tucson, p.1177. 
[210] Mumma, M.J. (1997), in Cosmovici, C.B., Bowyer, S., and Werthimer, D. (eds.), Astronomical and Biochemical Origins and the Search for Life in the Universe, Editrice Compositori of the Italian Physical Society, Bologna, p.121.

[211] Hagen, W., Allamandola, L.J. and Greenberg, J.M. (1979) Astrophys.Spa.Sci., 65, 215.

[212] Agarwal, V.K., et al (1985) Origins of Life, 16, 21.

[213] d'Hendecourt, L.B., Allamandola, L.J., Grim, R.J.A., and Greenberg, J.M. (1986) A\&A., 158, 119.

[214] Briggs, R., Ertem, G., Ferris, J.P., Greenberg, J.M., McCain, P.J., MendozaGomez, C.X., and Schutte, W. (1992) Orig.Life Evol.Biosphere, 22, 287.

[215] Gerakines, P.A., Schutte, W.A., Greenberg, J.M., and van Dishoek, E.F. (1995) $A \& A, 296,810$.

[216] Schutte, W.A., Allamandola, L.J., and Sandford, S.A. (1993) Icarus, 104, 118.

[217] Huebner, W.F., Boice, D.C., and Korth, A. (1989) Adv.Space Res., 9, 29.

[218] Bernstein, M.P., Sandford, S.A., Allamandola, L.J., and Chang, S. (1994) J.Phys.Chem., 98, 12206.

[219] Dickens, J.E., Irvine, W.M., DeVries, C.H. and Ohishi, M. (1997) Ap.J., 479, 307.

[220] Clemett, S., Maechling, C., Zare, R., Swan, P., and Walker, R. (1993) Science, 262, 721.

[221] Dworkin, J.P., Sandford, S.A., Allamandola, L.J., Deamer D., Gilette, J.S., and Zare, R.N. (2000) Orig.Life Evol.Biosphere, 30, 228.

[222] Bernstein, M.P., Sandford, S.A., Allamandola, L.J., Gillette, J.S., Clemett, S.J., and Zare, R.N. (1999) Science, 283, 1135.

[223] Sandford, S.A., Bernstein, M.P., Allamandola, L.J., Gilette, J.S., and Zare, R.N. (2000) Ap.J., 538, 691.

[224] Bernstein, M.P., Sandford, S.A., Allamandola, L.J. (2000) Ap.J., 542, 894.

[224] Bernstein, M.P., Dworkin, J.P., Sandford, S.A., and Allamandola, L.J. (2001) Met.\&Planet.Sci., 36, 351.

[225] Sellgren, K., Brooke, T.Y., Smith, R.G., and Geballe, T.R. (1995) Ap.J., 449, L69.

[226] Brooke, T.Y., Sellgren, K., and Geballe, T.R. (1999) Ap.J., 517, 883.

[227] Chiar, J.E., et al. (2000) Ap.J., 537, 749.

[228] Luisi, P.L. (1997), in Cosmovici, C.B., Bowyer, S., and Werthimer, D. (eds.), Astronomical and Biochemical Origins and the Search for Life in the Universe, Editrice Compositori of the Italian Physical Society, Bologna, p.461.

[229] DeDuve, C. (1997), in Cosmovici, C.B., Bowyer, S., and Werthimer, D. (eds.), Astronomical and Biochemical Origins and the Search for Life in the Universe, Editrice Compositori of the Italian Physical Society, Bologna, p.391.

[230] Deamer, D.W. (1985) Nature, 317, 792.

[231] Kerridge, J.F., Chang, S., and Shipp, R. (1987) Geochim.Cosmochim.Acta, 51, 2527.

[232] Messenger, S., Clemett, S.J., Keller, L.P., Thomas, K.L., Chillier, X.D.F., and Zare, R.N. (1995) Meteoritics, 30, 546.

[233] Hahn, J.H., Zenobi, R., Bada, J.L., and Zare, R.N. (1998) Science, 239, 1523.

[234] Cronin, J.R. and Chang, S. (1993), in Greenberg, J. M., Mendoza-Gómez, C. X., and Pirronello, V. (eds.), The Chemistry of Life's Origins, Kluwer Academic Pub., Dordrecht, p. 209. 
[235] Deamer, D.W. (1997) Microbio.\& Molec.Bio.Rev., 61, 239.

[236] Clemett, S., Maechling, C., Zare, R., Swan, P., and Walker, R. (1993) Science, 262, 721 .

[237] McKeegan, K.D., Walker, R.M., Zinner, E. (1985) Geochim.Cosmochim.Acta, 49, 1971.

[238] Cronin, J.R., Pizzarello, S., Epstein, S., and Krishnamurthy, R.V. (1993) Geochim.Cosmochim.Acta, 57, 4745.

[239] Chyba, C.F., Thomas, P.J., Brookshaw, L., and Sagan, C. (1990) Science, 249, 366. 